\title{
Two-loop rational terms in Yang-Mills theories
}

\author{
Jean-Nicolas Lang, ${ }^{a}$ Stefano Pozzorini, ${ }^{a}$ Hantian Zhang $^{a}$ and Max F. Zoller ${ }^{b}$ \\ ${ }^{a}$ Physik-Institut, Universität Zürich, \\ CH-8057 Zürich, Switzerland \\ ${ }^{b}$ Paul Scherrer Institut, \\ Forschungstrasse 111, CH-5232 Villigen PSI, Switzerland \\ E-mail: jlang@physik.uzh.ch, pozzorin@physik.uzh.ch, \\ hantian.zhang@physik.uzh.ch, max.zoller@psi.ch
}

ABSTRACT: Scattering amplitudes in $D$ dimensions involve particular terms that originate from the interplay of UV poles with the $(D-4)$-dimensional parts of loop numerators. Such contributions can be controlled through a finite set of process-independent rational counterterms, which make it possible to compute loop amplitudes with numerical tools that construct the loop numerators in four dimensions. Building on a recent study [1] of the general properties of two-loop rational counterterms, in this paper we investigate their dependence on the choice of renormalisation scheme. We identify a nontrivial form of scheme dependence, which originates from the interplay of mass and field renormalisation with the (D-4)-dimensional parts of loop numerators, and we show that it can be controlled through a new kind of one-loop counterterms. This guarantees that the two-loop rational counterterms for a given renormalisable theory can be derived once and for all in terms of generic renormalisation constants, which can be adapted a posteriori to any scheme. Using this approach, we present the first calculation of the full set of two-loop rational counterterms in Yang-Mills theories. The results are applicable to $\mathrm{SU}(\mathrm{N})$ and $\mathrm{U}(1)$ gauge theories coupled to $n_{\mathrm{f}}$ fermions with arbitrary masses.

KeYwords: Perturbative QCD, Precision QED, Scattering Amplitudes

ARXIV EPRINT: 2007.03713 


\section{Contents}

1 Introduction 1

2 Rational terms at one loop $\quad 3$

2.1 Notation and conventions 3

2.2 One-loop amplitudes with four-dimensional external momenta 3

2.3 One-loop amplitudes with $D$-dimensional external momenta 5

3 Rational terms at two loops $\quad 6$

$\begin{array}{ll}3.1 & \text { Notation for two-loop diagrams and subdiagrams }\end{array}$

$\begin{array}{lll}3.2 & \text { UV poles and rational parts at two loops } & 7\end{array}$

3.3 Sketch of the proof 8

4 Renormalisation scheme transformations $\quad 10$

$\begin{array}{lll}4.1 & \text { Multiplicative renormalisation } & 11\end{array}$

$\begin{array}{lll}4.2 & \text { Scale dependence and scheme transformations } & 13\end{array}$

$\begin{array}{ll}4.3 \text { Renormalisation formulas in } D_{\mathrm{n}}=4 \text { in a generic scheme } & 17\end{array}$

4.4 Nontrivial scheme dependence of two-loop rational terms 20

4.5 Full scheme dependence of two-loop rational terms 24

5 Two-loop rational counterterms for $\mathrm{SU}(\mathrm{N})$ and $\mathrm{U}(1)$ gauge theories 26

$\begin{array}{lll}5.1 & \text { Technical details of the calculations } & 26\end{array}$

$\begin{array}{ll}5.2 \text { Renormalised Lagrangian } & 28\end{array}$

$\begin{array}{lll}5.3 & \text { Rational counterterms } & 30\end{array}$

6 Conclusions $\quad 36$

$\begin{array}{ll}\text { A Tadpole expansions } & \mathbf{3 7}\end{array}$

$\begin{array}{lll}\text { A.1 Iterative tadpole decomposition } & 37\end{array}$

A.2 Power counting in $1 / \bar{q}_{i}$ and parametrisation dependence 41

A.3 Taylor expansion in the external momenta and masses 42

A.4 Taylor expansion with auxiliary one-loop counterterms 45

A.5 Invariance with respect to shifts of the loop momenta 46

$\begin{array}{ll}\text { B Renormalisation constants in the } \overline{\mathrm{MS}} \text { scheme } & 47\end{array}$ 


\section{Introduction}

Dimensional regularisation [2] is the most widely used method to regularise the ultraviolet (UV) and infrared (IR) singularities of scattering amplitudes in quantum-field theory. In this approach, loop amplitudes are computed in a continuous number $D$ of space-time dimensions, and the divergences of UV and IR kind assume the form of $1 /(D-4)$ poles. For this reason, the $(D-4)$-dimensional parts of loop momenta, metric tensors and Dirac matrices need to be manipulated with special care. In a computer algebra framework this is rather straightforward, while in the context of numerical algorithms, where algebraic quantities need to be implemented in an integer number of space-time dimensions, the consistent treatment of $(D-4)$-dimensional terms raises nontrivial technical and conceptual problems.

In the literature a variety of methods have been proposed that aim at restricting the calculation of loop amplitudes to an integer number of space-time dimensions [3-22]. At one loop, the most widely used method is based on the idea of splitting loop amplitudes into two parts according to the dimensionality $D_{\mathrm{n}}$ of the numerators of loop integrands. In this approach, loop amplitudes can be constructed by means of automated numerical algorithms in $D_{\mathrm{n}}=4$ dimensions, while the remaining $\left(D_{\mathrm{n}}-4\right)$-dimensional parts contribute only in combination with UV poles, and can be reconstructed a posteriori by means of processindependent rational counterterms [11,23-25]. This method is a key ingredient of the most efficient and flexible NLO automated tools on the market [26-29], and its extension to two loops is a natural strategy towards NNLO automation.

As a first step in this direction, recently it was shown that renormalised two-loop amplitudes in dimensional regularisation can be computed in terms of quantities in $D_{\mathrm{n}}=4$ dimensions and rational counterterms [1]. The relevant relation is encoded in the general formula

$$
\mathbf{R} \overline{\mathcal{A}}_{2, \Gamma}=\mathcal{A}_{2, \Gamma}+\sum_{\gamma}\left(\delta Z_{1, \gamma}+\delta \tilde{Z}_{1, \gamma}+\delta \mathcal{R}_{1, \gamma}\right) \cdot \mathcal{A}_{1, \Gamma / \gamma}+\delta Z_{2, \Gamma}+\delta \mathcal{R}_{2, \Gamma},
$$

where $\mathbf{R} \overline{\mathcal{A}}_{2, \Gamma}$ is the renormalised amplitude of a two-loop vertex function ${ }^{1}$ or a single two-loop diagram $\Gamma$ in $D$ dimensions. The corresponding one-loop subdiagrams and their complements are labelled $\gamma$ and $\Gamma / \gamma$, respectively, while $\mathcal{A}_{2, \Gamma}$ and $\mathcal{A}_{1, \Gamma / \gamma}$ denote the unrenormalised amplitudes of $\Gamma$ and $\Gamma / \gamma$ in $D_{\mathrm{n}}=4$ dimensions. The above formula features a similar structure as the well-known $\mathbf{R}$-operation [30-33], i.e. it involves the unrenormalised two-loop amplitude of $\Gamma$ in combination with one-loop counterterms associated with the UV divergences of the subdiagrams $\gamma$, as well as two-loop counterterms associated with the remaining local two-loop divergence. For the subtraction of UV divergences in $D_{\mathrm{n}}=4$ dimensions, the standard counterterms $\delta Z_{1, \gamma}$ and $\delta Z_{2, \Gamma}$ are supplemented by additional one-loop counterterms $\delta \tilde{Z}_{1, \gamma}$. Such extra UV counterterms are required only for quadratically divergent selfenergy subdiagrams $\gamma$ and are proportional to $\tilde{q}^{2} / \varepsilon$, where $\tilde{q}$ is the $(D-4)$-dimensional part of the loop momentum that flows through $\Gamma / \gamma$. The

\footnotetext{
${ }^{1}$ Here and throughout the paper by vertex, or vertex function, we mean any $N$-point function with $N \geq 2$ external lines.
} 
role of the remaining counterterms $\delta \mathcal{R}_{1}$ and $\delta \mathcal{R}_{2}$ in (1.1) is to reconstruct all parts of the renormalised two-loop amplitude that originate from the interplay of UV divergences with the $(D-4)$-dimensional terms in the loop numerator. The parts stemming from one-loop subdivergences are reconstructed through the well-known one-loop rational counterterms $\delta \mathcal{R}_{1}$, while the two-loop rational counterterms $\delta \mathcal{R}_{2}$ account for the parts stemming from the remaining local two-loop divergence.

The relation (1.1) holds for any process in any renormalisable theory, and, similarly as for the usual UV counterterms, also $\delta \tilde{Z}_{1}, \delta \mathcal{R}_{1}$ and $\delta \mathcal{R}_{2}$ are process-independent local counterterms that depend only on the theoretical model. Thus, once the $\delta \tilde{Z}_{1}, \delta \mathcal{R}_{1}$ and $\delta \mathcal{R}_{2}$ counterterms are available, their implementation amounts to a straightforward extension of the Feynman rules. A general method to derive the two-loop counterterms $\delta \mathcal{R}_{2}$ in any renormalisable model was presented in [1]. Technically, this procedure needs to be applied to all one-particle irreducible (1PI) vertex functions that involve a global UV divergence, and the required one- and two-loop integrals can be simplified using expansions that give rise to tadpole integrals with a single mass scale.

So far, the study of two-loop rational terms was restricted to effects of UV origin, assuming that IR divergences are either absent or are subtracted in a way that does not interfere with the rational terms. In fact, at one loop IR divergences do not give rise to any rational term [34]. However, the implications of IR divergences at two loops remain to be investigated. Another limitation of the original study of [1] lies in the fact that the derivation of the master formula (1.1) and the available $\delta \mathcal{R}_{2}$ counterterms for QED [1] are based on specific renormalisation schemes, namely the MS or $\overline{\mathrm{MS}}$ schemes.

In this paper - after a review in sections $2-3$ of the previous work on $\delta \mathcal{R}_{2}$ terms [1] the study of two-loop rational terms is extended in two new directions. First, in section 4 we demonstrate that the master formula (1.1) is valid for arbitrary renormalisation schemes, and we present a general analysis of the scheme dependence of $\delta \mathcal{R}_{2}$ counterterms of UV origin. To this end, we consider a finite multiplicative renormalisation of couplings, masses and fields, and we study its interplay with the projection of loop numerators to $D_{\mathrm{n}}=4$ dimensions. As we will show, these two operations do not commute at two loops, but the effect of their commutator can be encoded in a new set of scheme- and process-independent one-loop counterterms $\delta \hat{\mathcal{K}}_{1}$. This allows us to derive the general formulas (4.62)-(4.64), which describe the scheme dependence of $\delta \mathcal{R}_{2}$ counterterms as the result of the multiplicative renormalisation of the known $\delta \mathcal{R}_{1}$ counterterms plus a nontrivial part that can be written as a combination of one-loop renormalisation constants and $\delta \hat{\mathcal{K}}_{1}$ counterterms. In this way, the $\delta \mathcal{R}_{2}$ counterterms for a given theoretical model can be derived, once and for all, in the form of a linear combination of generic one-loop renormalisation constants, which can be adapted a posteriori to any desired renormalisation scheme.

The second main novelty of this paper is the first calculation of the full set of $\delta \mathcal{R}_{2}$ counterterms in Yang-Mills theories. As detailed in section 5, the relevant calculations are carried out in a generic renormalisation scheme and for a generic gauge group, while the results are presented in a form that is applicable both to $\mathrm{SU}(\mathrm{N})$ and $\mathrm{U}(1)$ gauge theories coupled to massless or massive fermions. The various tadpole expansions that have been used to compute and validate the required loop integrals are documented in detail in 
appendix A. There we present the expansion techniques that have already been used in [1], as well as a new optimised approach. Finally, for convenience of the reader, in appendix B we have collected all relevant UV renormalisation constants for the case of the $\overline{\mathrm{MS}}$ scheme.

\section{Rational terms at one loop}

In this section we introduce the conventions used throughout this paper and we briefly review the properties of one-loop rational terms following [1].

\subsection{Notation and conventions}

For the regularisation of UV divergences we use the 't Hooft-Veltman scheme [2], where external states are four-dimensional, while loop momenta as well as the metric tensors and Dirac matrices inside the loops live in $D=4-2 \varepsilon$ dimensions. For the decomposition of these objects into four-dimensional parts and $(D-4)$-dimensional remnants we use the notation $^{2}$

$$
\bar{q}^{\mu}=q^{\mu}+\tilde{q}^{\tilde{\mu}}, \quad \bar{\gamma}^{\mu}=\gamma^{\mu}+\tilde{\gamma}^{\tilde{\mu}}, \quad \bar{g}^{\bar{\mu} \bar{\nu}}=g^{\mu \nu}+\tilde{g}^{\tilde{\mu} \tilde{\nu}},
$$

where the bar and the tilde are used to mark, respectively, the $D$-dimensional and $(D-4)$ dimensional parts. To keep track of the dimensionality of loop numerators we use the parameter $D_{\mathrm{n}}$, which can assume the values $D$ or 4 . The case $D_{\mathrm{n}}=D$ corresponds to standard calculations in dimensional regularisation, while in $D_{\mathrm{n}}=4$ all loop numerators are projected to four dimensions keeping loop denominators in $D$ dimensions.

For the integration measure in loop-momentum space we use the shorthand

$$
\int \mathrm{d} \bar{q}=\mu_{0}^{2 \varepsilon} \int \frac{\mathrm{d}^{D} \bar{q}}{(2 \pi)^{D}}
$$

where $\mu_{0}$ is the scale of dimensional regularisation. For the renormalisation scale we use the symbol $\mu_{\mathrm{R}}$ and, at variance with [1], in this paper $\mu_{0}$ and $\mu_{\mathrm{R}}$ are treated as independent scales.

In the 't Hooft-Veltman scheme the renormalisation of UV divergences and the discussion of rational term of UV origin can be restricted to amputated 1PI vertex functions. For more details see [1].

\subsection{One-loop amplitudes with four-dimensional external momenta}

Let us consider the amplitude of a 1PI one-loop diagram $\Gamma$,

$$
\overline{\mathcal{A}}_{1, \Gamma}=\int \mathrm{d} \bar{q}_{1} \frac{\overline{\mathcal{N}}\left(\bar{q}_{1}\right)}{D_{0}\left(\bar{q}_{1}\right) \cdots D_{N-1}\left(\bar{q}_{1}\right)},
$$

with denominators

$$
D_{j}\left(\bar{q}_{1}\right)=\left(\bar{q}_{1}+p_{j}\right)^{2}-m_{j}^{2},
$$

\footnotetext{
${ }^{2}$ For more details see [1].
} 
where $p_{j}$ are combinations of four-dimensional external momenta. In $D_{\mathrm{n}}=D$ dimensions, the numerator $\overline{\mathcal{N}}\left(\bar{q}_{1}\right)$ can be split into

$$
\overline{\mathcal{N}}\left(\bar{q}_{1}\right)=\mathcal{N}\left(q_{1}\right)+\tilde{\mathcal{N}}\left(\bar{q}_{1}\right),
$$

where $\mathcal{N}\left(q_{1}\right)$ is the four-dimensional part, obtained by projecting the metric tensor, Dirac matrices and the loop momentum to four dimensions. The remnant part $\tilde{\mathcal{N}}\left(\bar{q}_{1}\right)$ is of $\mathcal{O}\left(\varepsilon, \tilde{q}_{1}\right)$ and will be referred to as the $(D-4)$-dimensional part of the numerator. As discussed in the following, its contribution can be controlled through a finite set of process-independent rational counterterms.

In view of the analysis of rational terms beyond one loop, it is convenient to discuss rational counterterms at the level of renormalised amplitudes. In the minimal subtraction (MS) scheme, for renormalised one-loop amplitudes we use the notation

$$
\mathbf{R} \overline{\mathcal{A}}_{1, \Gamma}=(1-\mathbf{K}) \overline{\mathcal{A}}_{1, \Gamma}=\overline{\mathcal{A}}_{1, \Gamma}+\delta Z_{1, \Gamma},
$$

where $\mathbf{K}$ is an operator that extracts the UV divergence according to the MS prescription, i.e. in the form of pure $1 / \varepsilon$ poles, and $\delta Z_{1, \Gamma}$ is the corresponding counterterm. Here and in the following, $\mathbf{R}$ and $\mathbf{K}$ should be understood as linear operators. Thus (2.6) is applicable both when $\Gamma$ is a single Feynman diagram or a set of diagrams, in which case the result is equivalent to the sum of the contributions of individual diagrams. This linearity property holds for all renormalisation identities in this paper.

At one loop, the renormalised amplitude can be constructed from quantities with $D_{\mathrm{n}}=4$ by means of the identity

$$
\mathbf{R} \overline{\mathcal{A}}_{1, \Gamma}=\mathcal{A}_{1, \Gamma}+\delta Z_{1, \Gamma}+\delta \mathcal{R}_{1, \Gamma}
$$

where $\mathcal{A}_{1, \Gamma}$ denotes the amplitude in $D_{\mathrm{n}}=4$ dimensions,

$$
\mathcal{A}_{1, \Gamma}=\int \mathrm{d} \bar{q}_{1} \frac{\mathcal{N}\left(q_{1}\right)}{D_{0}\left(\bar{q}_{1}\right) \cdots D_{N-1}\left(\bar{q}_{1}\right)},
$$

which can be computed with numerical tools that handle the numerator in four dimensions, while retaining the full $D$-dependence of the loop momentum in the denominator. The UV divergence of $\mathcal{A}_{1, \Gamma}$ is cancelled by the same $\delta Z_{1, \Gamma}$ counterterm as in (2.6), and the $\delta \mathcal{R}_{1, \Gamma}$ counterterm embodies the contribution of the $\tilde{\mathcal{N}}$-part of the numerator. At one loop, such $\delta \mathcal{R}_{1, \Gamma}$ counterterms originate only from the interplay of $\tilde{\mathcal{N}}$ with poles of UV type [34]. Thus, similarly as for UV counterterms, they can be derived once and for all for the set of UV divergent 1PI vertex functions [11, 23-25], where they take the form of homogeneous polynomials of degree $X$ in the external momenta $\left\{p_{k}\right\}$ and internal masses $\left\{m_{k}\right\}$, with $X$ being the degree of UV divergence of the vertex at hand.

In this paper we focus on the rational terms that originate from $\tilde{\mathcal{N}}$, for which we use the symbols $\delta \mathcal{R}_{L}$ at $L$ loops. Such terms will be referred to as $\tilde{\mathcal{N}}$ rational terms or simply rational terms. ${ }^{3}$

\footnotetext{
${ }^{3}$ Note that, in the literature on one-loop rational terms, the $\tilde{\mathcal{N}}$-terms of type $\delta \mathcal{R}_{1}$ are usually labelled $R_{2}$, while the label $R_{1}$ is used for one-loop rational terms stemming from the $(D-4)$-dimensional part of loop denominators. The latter kind of rational terms cannot be described by local counterterms, but can be controlled in a process-independent way through appropriate reduction algorithms in four dimensions (see e.g. [35, 36]) and will not be discussed in this paper.
} 

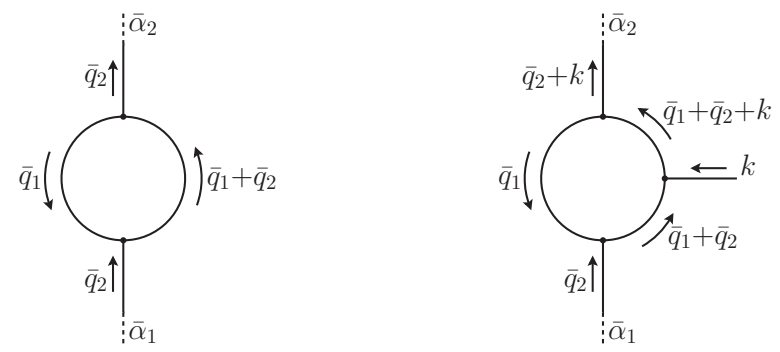

Figure 1. Examples of UV divergent one-loop subtopologies. The loop momentum $\bar{q}_{1}$ circulates inside the subdiagram, while the two external lines that are going to be embedded in a two-loop diagram depend on the $D$-dimensional loop momentum $\bar{q}_{2}$ and carry the Lorentz/Dirac indices $\bar{\alpha}_{1}, \bar{\alpha}_{2}$.

\subsection{One-loop amplitudes with $D$-dimensional external momenta}

An identity of type (2.7) is needed also for the one-loop subdiagrams of two-loop diagrams. As depicted in figure 1, this kind of one-loop (sub)diagrams involve an internal loop momentum $\bar{q}_{1}$ and an external loop momentum $\bar{q}_{2}$. Thus the relation (2.7) needs to be extended to the case of $D$-dimensional external kinematics.

For the renormalised amplitude of a generic one-loop subdiagram in $D_{\mathrm{n}}=D$ we have

$$
\mathbf{R} \overline{\mathcal{A}}_{1, \gamma}^{\bar{\alpha}}\left(\bar{q}_{2}\right)=(1-\mathbf{K}) \overline{\mathcal{A}}_{1, \gamma}^{\bar{\alpha}}\left(\bar{q}_{2}\right)=\overline{\mathcal{A}}_{1, \gamma}^{\bar{\alpha}}\left(\bar{q}_{2}\right)+\delta Z_{1, \gamma}^{\bar{\alpha}}\left(\bar{q}_{2}\right),
$$

where we explicitly indicate the dependence on the $D$-dimensional external loop momentum $\bar{q}_{2}$ and the multi-index $\bar{\alpha}=\left(\bar{\alpha}_{1}, \bar{\alpha}_{2}\right)$, which embodies the two Lorentz/Dirac indices associated with the two $\bar{q}_{2}$-dependent external lines (see figure 1 ). Since in $D_{\mathrm{n}}=D$ the momentum $\bar{q}_{2}$ has the same dimensionality in the loop numerator and denominator, the renormalised amplitudes (2.9) and (2.6) have the same form, and the corresponding UV counterterms are related through the simple replacements $q_{2} \rightarrow \bar{q}_{2}$ and $\alpha \rightarrow \bar{\alpha}$.

The extension of the identity (2.7) is more subtle, and the generalised formula for $D$-dimensional external kinematics reads [1]

$$
\mathbf{R} \overline{\mathcal{A}}_{1, \gamma}^{\bar{\alpha}}\left(\bar{q}_{2}\right)=\mathcal{A}_{1, \gamma}^{\alpha}\left(q_{2}\right)+\delta Z_{1, \gamma}^{\alpha}\left(q_{2}\right)+\delta \tilde{Z}_{1, \gamma}^{\alpha}\left(\tilde{q}_{2}\right)+\delta \mathcal{R}_{1, \gamma}^{\alpha}\left(q_{2}\right)+\mathcal{O}\left(\varepsilon, \tilde{q}_{2}\right)
$$

Here the amplitude in $D_{\mathrm{n}}=4$ dimensions on the r.h.s. is defined as

$$
\mathcal{A}_{1, \gamma}^{\alpha}\left(q_{2}\right)=\int \mathrm{d} \bar{q}_{1} \frac{\mathcal{N}^{\alpha}\left(q_{1}, q_{2}\right)}{D_{0}\left(\bar{q}_{1}, \bar{q}_{2}\right) \cdots D_{N-1}\left(\bar{q}_{1}, \bar{q}_{2}\right)}
$$

where all parts of the loop numerator, including $\alpha$ and $q_{2}$, are projected to four dimensions, while $\bar{q}_{2}$ is kept in $D$ dimensions in the loop denominator. The counterterms $\delta Z_{1, \gamma}$ and $\delta \mathcal{R}_{1, \gamma}$ on the r.h.s. of (2.10) are equivalent to the ones in (2.7), while $\delta \tilde{Z}_{1, \gamma}$ is a new UV counterterm that cannot be obtained from (2.7) via naive $q_{2} \rightarrow \bar{q}_{2}$ continuation. The $\delta \tilde{Z}_{1, \gamma}$ counterterm is required in order to cancel the UV divergence of the amplitude in $D_{\mathrm{n}}=4$ dimensions (2.11),

$$
\mathbf{K} \mathcal{A}_{1, \gamma}^{\alpha}\left(q_{2}\right)=-\delta Z_{1, \gamma}^{\alpha}\left(q_{2}\right)-\delta \tilde{Z}_{1, \gamma}^{\alpha}\left(\tilde{q}_{2}\right)
$$




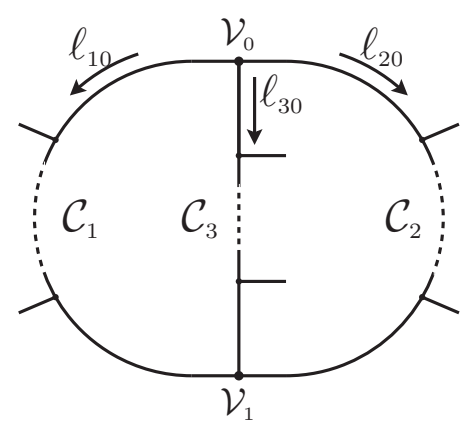

Figure 2. A generic irreducible two-loop diagram consists of two vertices, $\mathcal{V}_{0}, \mathcal{V}_{1}$, that connect three chains, $\mathcal{C}_{1}, \mathcal{C}_{2}, \mathcal{C}_{3}$, which contain, respectively, all propagators that depend on the loop momenta $q_{1}$, $q_{2}, q_{3}=-q_{1}-q_{2}$. For the sum of the loop momenta $\ell_{i 0}=q_{i}+p_{i 0}$, which flow out of $\mathcal{V}_{0}$, momentum conservation requires $\sum_{i} \ell_{i 0}=\sum_{i} p_{i 0}=k_{\text {ext }}$, where $k_{\text {ext }}$ is the external momentum that flows into $\mathcal{V}_{0}$. In practice, if $\mathcal{V}_{0}$ is a triple vertices (as in the picture) then $k_{\text {ext }}=0$ and all $p_{i 0}$ can be set equal to zero, while quartic vertices requires at least one non-vanishing $p_{i 0}$.

In renormalisable theories $\delta \tilde{Z}_{1, \gamma}$ is required only for quadratically divergent selfenergies, and its general form [1] is

$$
\delta \tilde{Z}_{1, \gamma}^{\alpha}\left(\tilde{q}_{2}\right)=v^{\alpha} \frac{\tilde{q}_{2}^{2}}{\varepsilon}
$$

where $v^{\alpha}$ is independent of $q_{2}$. The origin of this $\mathcal{O}\left(\tilde{q}_{2}^{2} / \varepsilon\right)$ counterterm lies in the fact that $\bar{q}_{2}$ is kept in $D$ dimensions in the loop denominator while it is projected to four dimensions in the numerator.

\section{Rational terms at two loops}

In this section we review the general analysis of two-loop rational terms presented in [1].

\subsection{Notation for two-loop diagrams and subdiagrams}

Two-loop amplitudes involve reducible and irreducible two-loop diagrams. The former can be factorised into one-loop parts, which generate, upon renormalisation, only one-loop rational terms [1]. Thus genuine two-loop rational terms originate only from irreducible diagrams. A generic irreducible two-loop diagram (see figure 2) consists of three chains, $\mathcal{C}_{1}, \mathcal{C}_{2}, \mathcal{C}_{3}$, that are connected to each other by two vertices, $\mathcal{V}_{0}, \mathcal{V}_{1}$. Each chain $\mathcal{C}_{i}$ includes a certain number $N_{i}$ of propagators that depend on the loop momentum $q_{i}$ and $N_{i}-1$ vertices. The loop momenta are related to each other by $\bar{q}_{1}+\bar{q}_{2}+\bar{q}_{3}=0$. The two-loop integral associated with a generic two-loop diagram $\Gamma$ has the form

$$
\overline{\mathcal{A}}_{2, \Gamma}=\left.\int \mathrm{d} \bar{q}_{1} \int \mathrm{d} \bar{q}_{2} \frac{\overline{\mathcal{N}}\left(\bar{q}_{1}, \bar{q}_{2}, \bar{q}_{3}\right)}{\mathcal{D}^{(1)}\left(\bar{q}_{1}\right) \mathcal{D}^{(2)}\left(\bar{q}_{2}\right) \mathcal{D}^{(3)}\left(\bar{q}_{3}\right)}\right|_{\bar{q}_{3}=-\bar{q}_{1}-\bar{q}_{2}},
$$

where each chain $\mathcal{C}_{i}$ contributes through the corresponding set of loop denominators,

$$
\mathcal{D}^{(i)}\left(\bar{q}_{i}\right)=D_{0}^{(i)}\left(\bar{q}_{i}\right) \cdots D_{N_{i}-1}^{(i)}\left(\bar{q}_{i}\right),
$$


with

$$
D_{a}^{(i)}\left(\bar{q}_{i}\right)=\bar{\ell}_{i a}^{2}-m_{i a}^{2}, \quad \text { and } \quad \bar{\ell}_{i a}=\bar{q}_{i}+p_{i a} .
$$

The form of the loop numerator is

$$
\overline{\mathcal{N}}\left(\bar{q}_{1}, \bar{q}_{2}, \bar{q}_{3}\right)=\bar{\Gamma}^{\bar{\alpha}_{1} \bar{\alpha}_{2} \bar{\alpha}_{3}}\left(\bar{q}_{1}, \bar{q}_{2}, \bar{q}_{3}\right) \overline{\mathcal{N}}_{\bar{\alpha}_{1}}^{(1)}\left(\bar{q}_{1}\right) \overline{\mathcal{N}}_{\bar{\alpha}_{2}}^{(2)}\left(\bar{q}_{2}\right) \overline{\mathcal{N}}_{\bar{\alpha}_{3}}^{(3)}\left(\bar{q}_{3}\right),
$$

where the parts $\overline{\mathcal{N}}_{\bar{\alpha}_{i}}^{(i)}\left(\bar{q}_{i}\right)$ associated to each chain $\mathcal{C}_{i}$ are connected through the multi-indices

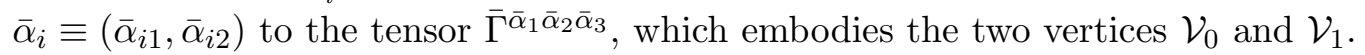

Irreducible two-loop diagrams $\Gamma$ involve three one-loop subdiagrams $\gamma_{i}$, which result from $\Gamma$ by truncating the chain $\mathcal{C}_{i}$. More precisely, each partition $i \mid j k$ of 123 defines a subdiagram $\gamma_{i}$ that contains the chains $\mathcal{C}_{j}$ and $\mathcal{C}_{k}$. Its amplitude reads

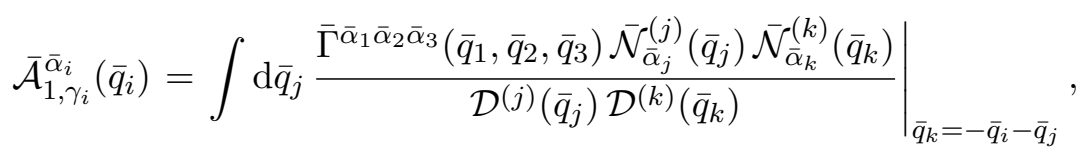

where $\bar{q}_{i}$ plays the role of external momentum, and $\bar{\alpha}_{i}$ connects $\gamma_{i}$ to its complement $\Gamma / \gamma_{i}$, which contains the chain $\mathcal{C}_{i}$, and is derived from $\Gamma$ by shrinking $\gamma_{i}$ to a vertex.

Similarly as in (2.5), the two-loop numerator can be split into four-dimensional and $(D-4)$-dimensional parts as

$$
\overline{\mathcal{N}}\left(\bar{q}_{1}, \bar{q}_{2}, \bar{q}_{3}\right)=\mathcal{N}\left(q_{1}, q_{2}, q_{3}\right)+\tilde{\mathcal{N}}\left(\bar{q}_{1}, \bar{q}_{2}, \bar{q}_{3}\right) .
$$

As discussed below, explicit two-loop calculations can be restricted to the four-dimensional $\mathcal{N}$ contribution, while all $\tilde{\mathcal{N}}$-terms can be reconstructed by means of rational counterterms.

\subsection{UV poles and rational parts at two loops}

In general, two-loop amplitudes involve subdivergences and additional local two-loop divergences. These two kinds of divergences can be subtracted by means of the so-called R-operation [30-33]. For a single two-loop diagram or a full two-loop vertex function $\Gamma$, the subtracted amplitude has the form

$$
\mathbf{R} \overline{\mathcal{A}}_{2, \Gamma}=\overline{\mathcal{A}}_{2, \Gamma}+\sum_{\gamma} \delta Z_{1, \gamma} \cdot \overline{\mathcal{A}}_{1, \Gamma / \gamma}+\delta Z_{2, \Gamma},
$$

where $\overline{\mathcal{A}}_{2, \Gamma}$ is the unrenormalised two-loop amplitude in $D$ dimensions. The second term on the r.h.s. subtracts all relevant subdivergences. When $\Gamma$ is a single two-loop diagram the sum involves the three one-loop subdiagrams $\gamma=\gamma_{1}, \gamma_{2}, \gamma_{3}$ of $\Gamma$. The corresponding UV divergences are subtracted by the counterterms

$$
\delta Z_{1, \gamma_{i}}=-\mathbf{K} \overline{\mathcal{A}}_{1, \gamma_{i}},
$$

and their insertion into the complementary one-loop diagrams $\Gamma / \gamma_{i}$ read

$$
\delta Z_{1, \gamma_{i}} \cdot \overline{\mathcal{A}}_{1, \Gamma / \gamma_{i}}=\int \mathrm{d} \bar{q}_{i} \delta Z_{1, \gamma_{i}}^{\bar{\alpha}_{i}}\left(\bar{q}_{i}\right) \frac{\overline{\mathcal{N}}_{\bar{\alpha}_{i}}^{(i)}\left(\bar{q}_{i}\right)}{\mathcal{D}^{(i)}\left(\bar{q}_{i}\right)} .
$$


The counterterm $\delta Z_{2, \Gamma}$ in (3.7) subtracts the local two-loop divergence that is left after subtraction of the subdivergences. If $\Gamma$ is a full two-loop vertex function, the identity (3.7) can be applied at the level of the individual two-loop diagrams that contribute to $\Gamma$ by handling $\mathbf{R}$ as a linear operator. Alternatively, (3.7) can be directly applied to the full vertex $\Gamma$. In this case, $\delta Z_{2, \Gamma}$ corresponds to the full UV counterterm for $\Gamma$, and $\delta Z_{1, \gamma}$ are the complete one-loop counterterms for the vertices $\gamma$ that can be inserted into the oneloop vertex function $\mathcal{A}_{1, \Gamma}$, while $\delta Z_{1, \gamma} \cdot \overline{\mathcal{A}}_{1, \Gamma / \gamma}$ embodies all possible insertions of a certain counterterm $\delta Z_{1, \gamma}$ into the various one-loop diagrams that contribute to $\mathcal{A}_{1, \Gamma}$.

The presence of a UV divergence in a subdiagram $\gamma_{i}$ can be identified by means of the degree of (sub)divergence

$$
X\left(\gamma_{i}\right)=X_{j k}(\Gamma)=4+U_{j}(\Gamma)+U_{k}(\Gamma)+\sum_{a=0}^{1} Y_{a}(\Gamma)
$$

where $U_{m}(\Gamma)$ denotes the maximum power in $q_{m}$ along the chain $\mathcal{C}_{m}$, and $Y_{a}(\Gamma)$ is the generic power in $q$ of the vertex $\mathcal{V}_{a}$. Subdiagrams with $X\left(\gamma_{i}\right) \geq 0$ are UV divergent. The remaining local two-loop divergences can be identified by means of the global degree of divergence

$$
X(\Gamma)=8+\sum_{i=1}^{3} U_{i}(\Gamma)+\sum_{a=0}^{1} Y_{a}(\Gamma)
$$

which corresponds to the total loop-momentum power of the full two-loop diagram. Globally divergent diagrams, i.e. diagrams with $X(\Gamma) \geq 0$, involve local divergences.

As demonstrated in [1], the renormalised two-loop amplitude (3.7) in $D_{\mathrm{n}}=D$ dimensions can be expressed in terms of amplitudes in $D_{\mathrm{n}}=4$ dimensions plus appropriate rational counterterms. The corresponding master formula reads

$$
\mathbf{R} \overline{\mathcal{A}}_{2, \Gamma}=\mathcal{A}_{2, \Gamma}+\sum_{\gamma}\left(\delta Z_{1, \gamma}+\delta \tilde{Z}_{1, \gamma}+\delta \mathcal{R}_{1, \gamma}\right) \cdot \mathcal{A}_{1, \Gamma / \gamma}+\delta Z_{2, \Gamma}+\delta \mathcal{R}_{2, \Gamma}
$$

and is illustrated in figure 3. The first term on the r.h.s. is the unrenormalised two-loop amplitude in $D_{\mathrm{n}}=4$ dimensions, which corresponds to $\overline{\mathcal{A}}_{2, \Gamma}$ with the $\tilde{\mathcal{N}}$-part of the numerator (3.6) set to zero. The second term contains all required one-loop counterterms - see (2.10) - for the cancellation of the UV poles of the subdiagrams $\gamma$ and for the reconstruction of the associated rational parts. As for the remaining two-loop counterterms, $\delta Z_{2, \Gamma}$ is the same UV counterterm as in (3.7), while the rational counterterm $\delta \mathcal{R}_{2, \Gamma}$ reconstructs all remaining contributions of order $\varepsilon^{-1}$ and $\varepsilon^{0}$ that originate form the interplay of the $\tilde{\mathcal{N}}$-part of the numerator with local UV divergences.

As demonstrated in [1], the $\delta \mathcal{R}_{2, \Gamma}$ terms are process-independent local counterterms, and can be computed once and for all in terms of tadpole integrals.

\subsection{Sketch of the proof}

In the following we review the key aspects of the proof of the master formula (3.12) and we outline how to compute $\delta \mathcal{R}_{2}$ terms from tadpole integrals with one mass scale. 


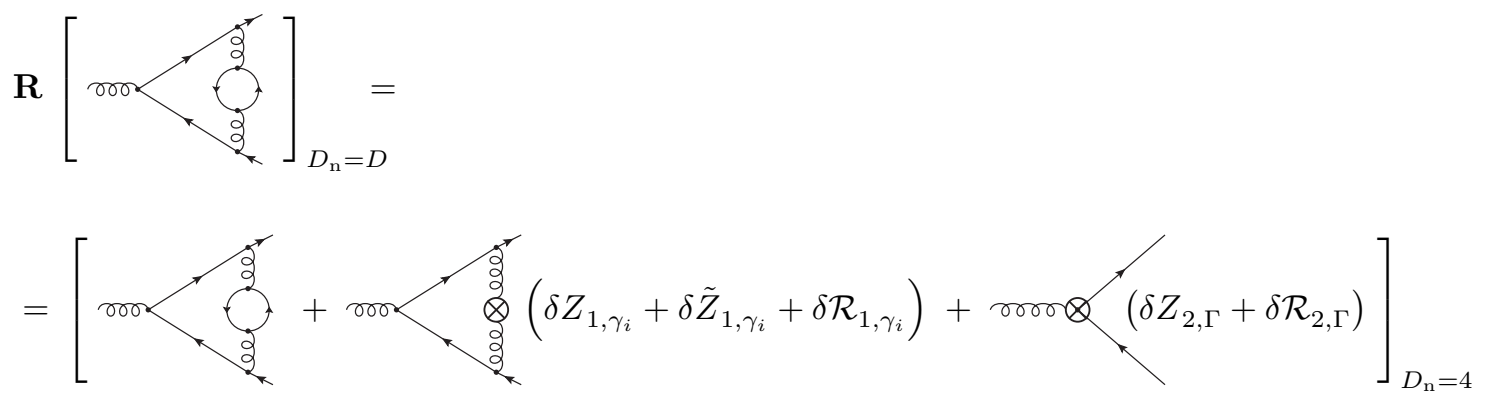

Figure 3. Graphical representation of the master formula (3.12) for the case of a globally divergent two-loop QCD diagram with a single subdivergence.

In renormalisable theories, two-loop diagrams $\Gamma$ with $X(\Gamma)<0$ involve at most one divergent subdiagram $\gamma$, for which, according to (2.10),

$$
\overline{\mathcal{A}}_{1, \gamma}+\delta Z_{1, \gamma}=\mathcal{A}_{1, \gamma}+\delta Z_{1, \gamma}+\delta \tilde{Z}_{1, \gamma}+\delta \mathcal{R}_{1, \gamma}+\mathcal{O}\left(\varepsilon, \tilde{q}_{2}^{2}\right)
$$

where $\bar{q}_{2}$ is the loop momentum that circulates through the complementary part $\Gamma / \gamma$ of the two-loop diagram. Using this identity one can show that, up to negligible $\mathcal{O}(\varepsilon)$ terms,

$$
\overline{\mathcal{A}}_{2, \Gamma}+\delta Z_{1, \gamma} \cdot \overline{\mathcal{A}}_{1, \Gamma / \gamma}=\mathcal{A}_{2, \Gamma}+\left(\delta Z_{1, \gamma}+\delta \tilde{Z}_{1, \gamma}+\delta \mathcal{R}_{1, \gamma}\right) \cdot \mathcal{A}_{1, \Gamma / \gamma}
$$

which is equivalent to the master formula (3.12) with

$$
\delta Z_{2, \Gamma}=0 \quad \text { and } \quad \delta \mathcal{R}_{2, \Gamma}=0 \quad \text { for } \quad X(\Gamma)<0 .
$$

This means that two-loop rational terms $\delta \mathcal{R}_{2, \Gamma}$ occur only in the presence of a local divergence. Therefore they can be determined, once and for all, by inverting the master formula (3.12), i.e. by computing

$$
\delta \mathcal{R}_{2, \Gamma}=\overline{\mathcal{A}}_{2, \Gamma}-\mathcal{A}_{2, \Gamma}+\sum_{\gamma} \delta Z_{1, \gamma} \cdot \overline{\mathcal{A}}_{1, \Gamma / \gamma}-\sum_{\gamma}\left(\delta Z_{1, \gamma}+\delta \tilde{Z}_{1, \gamma}+\delta \mathcal{R}_{1, \gamma}\right) \cdot \mathcal{A}_{1, \Gamma / \gamma}
$$

for all 1PI amputated vertex functions $\Gamma$ with $X(\Gamma) \geq 0$. The fact that $\delta \mathcal{R}_{2, \Gamma}$ terms originate only from local UV divergences makes it possible to express (3.16) in terms of tadpole integrals [1]. Technically, this is achieved by decomposing two-loop diagrams into two parts,

$$
\overline{\mathcal{A}}_{2, \Gamma}=\overline{\mathcal{A}}_{2, \Gamma_{\text {tad }}}+\overline{\mathcal{A}}_{2, \Gamma_{\mathrm{rem}}},
$$

where $\Gamma_{\text {tad }}$ is constructed through a systematic expansion that embodies the local divergence of $\Gamma$ in the form of tadpole integrals. Here we focus on the general properties of such tadpole expansions, while their explicit form and various possible optimisations are discussed in detail in appendix A. By construction, the remnant of the expansion in (3.17) is free from local divergences, i.e. ${ }^{4}$

$$
X\left(\Gamma_{\text {rem }}\right)<0 .
$$

\footnotetext{
${ }^{4}$ This identity should hold in $D_{\mathrm{n}}=D$ dimensions, i.e. both for the four-dimensional and the $\left(D_{\mathrm{n}}-4\right)$ dimensional parts of the loop numerator, where terms of $\mathcal{O}\left(\tilde{q}_{i}\right)$ and $\mathcal{O}\left(\varepsilon q_{i}\right)$ should be counted on the same footing as $\mathcal{O}\left(q_{i}\right)$. The same holds also for (3.21).
} 
Therefore, according to (3.15) the $\delta \mathcal{R}_{2}$ terms of $\Gamma$ arise only from its $\Gamma_{\text {tad }}$ part and can be calculated with the formula

$$
\begin{aligned}
\delta \mathcal{R}_{2, \Gamma}= & \overline{\mathcal{A}}_{2, \Gamma_{\mathrm{tad}}}-\mathcal{A}_{2, \Gamma_{\mathrm{tad}}}+\sum_{\gamma} \delta Z_{1, \gamma_{\mathrm{tad}}} \cdot \overline{\mathcal{A}}_{1, \Gamma_{\mathrm{tad}} / \gamma_{\mathrm{tad}}} \\
& -\sum_{\gamma}\left(\delta Z_{1, \gamma_{\mathrm{tad}}}+\delta \tilde{Z}_{1, \gamma_{\mathrm{tad}}}+\delta \mathcal{R}_{1, \gamma_{\mathrm{tad}}}\right) \cdot \mathcal{A}_{1, \Gamma_{\mathrm{tad}} / \gamma_{\mathrm{tad}}}
\end{aligned}
$$

Here $\gamma_{\text {tad }}$ are the various subdiagrams of $\Gamma_{\text {tad }}$, and $\delta Z_{1, \gamma_{\text {tad }}}, \delta \tilde{Z}_{1, \gamma_{\text {tad }}}$ and $\delta \mathcal{R}_{1, \gamma_{\text {tad }}}$ are the one-loop counterterms associated with the corresponding subdivergences. The latter do not need to coincide with the subdivergences of the original diagram $\Gamma$. However, as discussed in appendix A, the tadpole decomposition (3.17) can be implemented in such a way that $\Gamma_{\text {tad }}$ and $\Gamma$ have the same subdivergences. More precisely, one can require that the difference between the corresponding subdiagrams,

$$
\overline{\mathcal{A}}_{1, \delta \gamma_{\text {tad }}}=\overline{\mathcal{A}}_{1, \gamma}-\overline{\mathcal{A}}_{1, \gamma_{\text {tad }}}
$$

is free from UV divergences, i.e.

$$
X\left(\delta \gamma_{\text {tad }}\right)<0
$$

If this condition is fulfilled, then all subdiagrams of $\Gamma_{\text {tad }}$ and $\Gamma$ have identical UV poles and rational parts, i.e.

$$
\delta Z_{1, \gamma_{\mathrm{tad}}}=\delta Z_{1, \gamma}, \quad \delta \tilde{Z}_{1, \gamma_{\mathrm{tad}}}=\delta \tilde{Z}_{1, \gamma}, \quad \delta \mathcal{R}_{1, \gamma_{\mathrm{tad}}}=\delta \mathcal{R}_{1, \gamma}
$$

The above considerations apply to single two-loop diagrams $\Gamma$ with related subdiagrams $\gamma$, but can be directly extended - as discussed after (3.9) — to the case where $\Gamma$ and $\gamma$ are full vertex functions.

Based on the formula (3.19) and the general properties of the tadpole expansion (see appendix A.1) one can show [1] that the $\delta \mathcal{R}_{2, \Gamma}$ counterterms associated with 1 PI vertex functions take the form of homogeneous polynomials of degree $X(\Gamma)$ in the external momenta $\left\{p_{i a}\right\}$ and internal masses $\left\{m_{i a}\right\}$.

\section{Renormalisation scheme transformations}

The goal of this section is to generalise the master formulas (2.7) and (3.12), which have been derived in the MS scheme and its $\overline{\mathrm{MS}}$ variant, to any renormalisation scheme. As we will see, the form of the master formulas is independent of the renormalisation scheme, i.e. we will demonstrate that the renormalised one- and two-loop amplitudes in a generic scheme $X$ fulfil the relations

$$
\begin{aligned}
& \mathbf{R}^{(X)} \overline{\mathcal{A}}_{1, \Gamma}=\mathcal{A}_{1, \Gamma}+\delta Z_{1, \Gamma}^{(X)}+\delta \mathcal{R}_{1, \Gamma}^{(X)} \\
& \mathbf{R}^{(X)} \overline{\mathcal{A}}_{2, \Gamma}=\mathcal{A}_{2, \Gamma}+\sum_{\gamma}\left(\delta Z_{1, \gamma}^{(X)}+\delta \tilde{Z}_{1, \gamma}^{(X)}+\delta \mathcal{R}_{1, \gamma}^{(X)}\right) \cdot \mathcal{A}_{1, \Gamma / \gamma}+\delta Z_{2, \Gamma}^{(X)}+\delta \mathcal{R}_{2, \Gamma}^{(X)}
\end{aligned}
$$


where $\delta Z_{k}^{(X)}$ are the UV counterterms in the scheme $X$. As we will show, the remaining $k$-loop counterterms $\delta \tilde{Z}_{k}^{(X)}$ and rational terms $\delta \mathcal{R}_{k}^{(X)}$ are related to the corresponding objects in the $\overline{\mathrm{MS}}$ scheme through transformations that involve only lower-order counterterms and rational terms. Actually, apart from a trivial scale dependence, $\delta \mathcal{R}_{1}$ and $\delta \tilde{Z}_{1}$ are scheme independent. As for the $\delta \mathcal{R}_{2}$ terms, it turns out — see (4.62) and (4.63) that their transformation amounts to a finite renormalisation of the corresponding $\delta \mathcal{R}_{1}$ terms plus a nontrivial contribution that can be expressed - see (4.64) and (4.100) - as a combination of one-loop renormalisation constants and auxiliary one-loop counterterms.

Note that, for consistency with the original formulation [1], the generalised master formulas (4.1)-(4.2) are expressed in the language of the $\mathbf{R}$-operation, where one-loop subdivergences are subtracted via insertion of UV counterterms $\delta Z_{1, \gamma}^{(X)}$ at the level of subdiagrams. This calls for an operational definition of such $\delta Z_{1, \gamma}^{(X)}$ insertions in $D_{\mathrm{n}}=4$ dimensions in a generic scheme $X$. Motivated by the practical goal of expressing renormalised amplitudes in terms of loop integrals with four-dimensional numerators, we adopt the definition

$$
\sum_{\gamma} \delta Z_{1, \gamma}^{(X)} \cdot \mathcal{A}_{1, \Gamma / \gamma}=\left[\sum_{\gamma} \delta Z_{1, \gamma}^{(X)} \cdot \overline{\mathcal{A}}_{1, \Gamma / \gamma}\right]_{D_{\mathrm{n}}=4},
$$

i.e. the $\delta Z_{1, \gamma}^{(X)}$ insertions on the r.h.s. of (4.2) should be understood as a standard UV subtraction in $D_{\mathrm{n}}=D$ dimensions with a subsequent projection to $D_{\mathrm{n}}=4$. Actually, in a generic renormalisation scheme the subtraction of UV divergences is controlled through the multiplicative renormalisation of parameters and fields at the level of the Lagrangian. Thus, contrary to the case of the minimal subtraction scheme, the prescription (4.3) cannot be applied at the level of individual two-loop diagrams and their subdiagrams, but should be understood at the level of the full sets of (sub)diagrams that are associated with the renormalisation of a certain parameter or field. In practice (4.3) can be implemented, as detailed in (4.67)-(4.68), via multiplicative renormalisation of the amplitude $\overline{\mathcal{A}}_{1, \Gamma}$ of a full $n$-point vertex function $\Gamma$ with a posteriori projection to $D_{\mathrm{n}}=4$.

In order to derive the above mentioned properties, in sections 4.1-4.2 we first introduce an appropriate scheme-transformations formalism for amplitudes in $D_{\mathrm{n}}=D$ dimensions. The connection to amplitudes in $D_{\mathrm{n}}=4$ dimensions is established in sections 4.3-4.4, and the main results for the scheme dependence of rational terms are presented in (4.62)-(4.63) and in section 4.5.

\subsection{Multiplicative renormalisation}

Let us consider a generic renormalisable theory with a certain set of fields $\left\{\varphi_{j}\right\}$ and a set of parameters $\left\{\theta_{i}\right\}=\{\alpha, \lambda, m\}$. For simplicity we restrict ourselves to a single coupling constant $\alpha$, a gauge-fixing parameter $\lambda$, and a mass parameter $m$, but the formalism introduced in the following is applicable to any number of parameters.

To define a generic renormalisation scheme we adopt the multiplicative renormalisation approach, where the cancellation of UV divergences is controlled through the identities

$$
\varphi_{j, 0}=\left(\mathcal{Z}_{\varphi_{j}}^{(X)}\right)^{1 / 2} \varphi_{i, X}, \quad \theta_{i, 0}=\mathcal{Z}_{\theta_{i}}^{(X)} \theta_{i, X} \quad \text { for } \quad \theta_{i}=\alpha, \lambda, m
$$


where $\varphi_{j, 0}$ and $\theta_{i, 0}$ denote the scheme-independent bare fields and parameters, while $\varphi_{j, X}$ and $\theta_{i, X}$ are their renormalised counterparts. The label $X$ corresponds to a generic renormalisation scheme, which may be the $\overline{\mathrm{MS}}$ scheme, the on-shell scheme, or any other scheme. For the perturbative expansion of the renormalisation constants we use the notation

$$
\mathcal{Z}_{\chi}^{(X)}=1+\sum_{k=1}^{\infty} \delta \mathcal{Z}_{k, \chi}^{(X)} \quad \text { for } \quad \chi=\alpha, \lambda, m, \varphi_{j}
$$

where the index $k$ is the order in the coupling constant, i.e. $\delta \mathcal{Z}_{k, \chi}^{(X)} \propto \alpha_{X}^{k}$. Note that (4.4)(4.5) imply that

$$
\frac{\alpha_{0}}{\alpha_{X}}=1+\mathcal{O}\left(\alpha_{X}\right)
$$

The renormalisation formula for a scattering amplitude reads

$$
\mathbf{R}^{(X)} \overline{\mathcal{A}}_{\Gamma}\left(\left\{\theta_{i, X}\right\}\right)=\left[\prod_{j}\left(\mathcal{Z}_{\varphi_{j}}^{(X)}\right)^{1 / 2}\right] \overline{\mathcal{A}}_{\Gamma}\left(\left\{\theta_{i, 0}\right\}\right),
$$

where the index $j$ runs over all external legs, and the scattering amplitude $\mathcal{A}_{\Gamma}$ includes terms of any order in the coupling constant. The combined effect of field and parameter renormalisation in (4.7) can be cast in the form

$$
\mathbf{R}^{(X)} \overline{\mathcal{A}}_{\Gamma}\left(\left\{\theta_{i, X}\right\}\right)=\sum_{k=0}^{\infty} D_{k}^{(X)} \overline{\mathcal{A}}_{\Gamma}\left(\left\{\theta_{i, X}\right\}\right),
$$

where $D_{k}^{(X)}$ are operators of order $\alpha_{X}^{k}$ that contain combinations of renormalisation constants and derivatives with respect to the corresponding parameters, $\theta_{i}=\alpha, \lambda, m$. Up to second order they read

$$
\begin{aligned}
D_{0}^{(X)}= & 1 \\
D_{1}^{(X)}= & \sum_{i} \delta \mathcal{Z}_{1, \theta_{i}}^{(X)} \theta_{i} \frac{\partial}{\partial \theta_{i}}+\sum_{j} \frac{1}{2} \delta \mathcal{Z}_{1, \varphi_{j}}^{(X)} \\
D_{2}^{(X)}= & \sum_{i} \delta \mathcal{Z}_{2, \theta_{i}}^{(X)} \theta_{i} \frac{\partial}{\partial \theta_{i}}+\frac{1}{2} \sum_{i, k} \delta \mathcal{Z}_{1, \theta_{i}}^{(X)} \delta \mathcal{Z}_{1, \theta_{k}}^{(X)} \theta_{i} \theta_{j} \frac{\partial^{2}}{\partial \theta_{i} \partial \theta_{j}}+\frac{1}{2} \sum_{i} \sum_{j} \delta \mathcal{Z}_{1, \varphi_{j}}^{(X)} \delta \mathcal{Z}_{1, \theta_{i}}^{(X)} \theta_{i} \frac{\partial}{\partial \theta_{i}} \\
& +\frac{1}{4} \sum_{i} \sum_{j<i} \delta \mathcal{Z}_{1, \varphi_{i}}^{(X)} \delta \mathcal{Z}_{1, \varphi_{j}}^{(X)}+\sum_{j}\left[\frac{1}{2} \delta \mathcal{Z}_{2, \varphi_{j}}^{(X)}-\frac{1}{8}\left(\delta \mathcal{Z}_{1, \varphi_{j}}^{(X)}\right)^{2}\right]
\end{aligned}
$$

Let us now consider the interplay of (4.8) with the perturbative expansion of the unrenormalised scattering amplitude,

$$
\overline{\mathcal{A}}_{\Gamma}\left(\left\{\theta_{i, X}\right\}\right)=\sum_{k=0}^{\infty} \overline{\mathcal{A}}_{k, \Gamma}\left(\left\{\theta_{i, X}\right\}\right),
$$

where $\overline{\mathcal{A}}_{k, \Gamma}$ denotes the $k$-loop contribution, and we assume that $\overline{\mathcal{A}}_{k, \Gamma} \propto \alpha_{X}^{p+k}$. Combining (4.8) with (4.12) we can write the $n$-loop contribution to the renormalised amplitude 


$$
\mathbf{R}^{(X)} \overline{\mathcal{A}}_{n, \Gamma}=\sum_{m=0}^{n} D_{n-m}^{(X)} \overline{\mathcal{A}}_{m, \Gamma},
$$

or, more explicitly, up to two loops

$$
\begin{aligned}
& \mathbf{R}^{(X)} \overline{\mathcal{A}}_{0, \Gamma}=\mathcal{A}_{0, \Gamma} \\
& \mathbf{R}^{(X)} \overline{\mathcal{A}}_{1, \Gamma}=\overline{\mathcal{A}}_{1, \Gamma}+D_{1}^{(X)} \mathcal{A}_{0, \Gamma}, \\
& \mathbf{R}^{(X)} \overline{\mathcal{A}}_{2, \Gamma}=\overline{\mathcal{A}}_{2, \Gamma}+D_{1}^{(X)} \overline{\mathcal{A}}_{1, \Gamma}+D_{2}^{(X)} \mathcal{A}_{0, \Gamma} .
\end{aligned}
$$

Note that here we assume that the tree amplitude is free from $(D-4)$-dimensional parts, i.e. $\overline{\mathcal{A}}_{0, \Gamma}=\mathcal{A}_{0, \Gamma}$.

\subsection{Scale dependence and scheme transformations}

The subtraction of UV singularities is associated with a renormalisation scale $\mu_{\mathrm{R}}$, which enters the renormalisation formulas through a dimensionless ratio of the form

$$
t_{X}=\frac{S_{X} \mu_{0}^{2}}{\mu_{\mathrm{R}}^{2}},
$$

where $\mu_{0}$ and $\mu_{\mathrm{R}}$ are, respectively, the 't Hooft scale of dimensional regularisation and the renormalisation scale. Note that $\mu_{\mathrm{R}}$ can be introduced "by hand" as part of the technical prescription for the renormalisation of $\alpha$, like in the MS and $\overline{\mathrm{MS}}$ schemes, or it can arise from a physical renormalisation condition, like in on-shell schemes, in which case $\mu_{\mathrm{R}}$ is typically a physical mass or energy scale. The term $S_{X}$ in (4.17) is a scheme-dependent factor, and its explicit values in the MS and $\overline{\mathrm{MS}}$ schemes are indicated in (4.27) and (4.30).

The scale dependence can be implemented in two different ways, which can be regarded as two equivalent formulations of a scale-dependent renormalisation scheme. These two approaches will be referred to as scheme $X_{0}$ and scheme $X$. The first approach corresponds to renormalisation identities of the form

$$
\varphi_{j, 0}=\left(\mathcal{Z}_{\varphi_{j}}^{\left(X_{0}\right)}\right)^{1 / 2} \varphi_{i, X_{0}}, \quad \alpha_{0}=t_{X}^{-\varepsilon} \mathcal{Z}_{\alpha}^{\left(X_{0}\right)} \alpha_{X_{0}}\left(\mu_{\mathrm{R}}^{2}\right), \quad \theta_{i, 0}=\mathcal{Z}_{\theta_{i}}^{\left(X_{0}\right)} \theta_{i, X_{0}}\left(\mu_{\mathrm{R}}^{2}\right)
$$

for $\theta_{i}=\lambda, m$. Here the scale dependence is entirely controlled through the factor $t_{X}^{-\varepsilon}$ in the renormalisation of the coupling constant, while the renormalisation constants $\mathcal{Z}_{\chi}^{\left(X_{0}\right)}$ are free from any explicit scale dependence. Their perturbative expansion has the same form as (4.5), i.e.

$$
\mathcal{Z}_{\chi}^{\left(X_{0}\right)}=1+\sum_{k=1}^{\infty} \delta \mathcal{Z}_{k, \chi}^{\left(X_{0}\right)} \quad \text { for } \quad \chi=\alpha, \lambda, m, \varphi_{j}
$$

At variance with (4.6), the above renormalisation identities imply

$$
\frac{\alpha_{0}}{\alpha_{X_{0}}}=t_{X}^{-\varepsilon}+\mathcal{O}\left(\alpha_{X}\right)
$$


and are thus inconsistent with the formalism of section 4.1. This issue can be circumvented by converting the above renormalisation identities into the form (4.4)-(4.5). This can be achieved by rescaling the coupling constant as

$$
\alpha_{X_{0}}\left(\mu_{\mathrm{R}}^{2}\right)=t_{X}^{\varepsilon} \alpha_{X}\left(\mu_{\mathrm{R}}^{2}\right)
$$

while keeping all other parameters and fields unchanged. This finite renormalisation ${ }^{5}$ turns the scheme $X_{0}$ into the equivalent scheme $X$, and the resulting renormalisation identities read

$$
\varphi_{j, 0}=\left(\mathcal{Z}_{\varphi_{j}}^{(X)}\right)^{1 / 2} \varphi_{i, X}, \quad \theta_{i, 0}=\mathcal{Z}_{\theta_{i}}^{(X)} \theta_{i, X}\left(\mu_{\mathrm{R}}^{2}\right) \quad \text { for } \quad \theta_{i}=\alpha, \lambda, m
$$

where

$$
\mathcal{Z}_{\chi}^{(X)}=1+\sum_{k=1}^{\infty} \delta \mathcal{Z}_{k, \chi}^{(X)} \quad \text { for } \quad \chi=\alpha, \lambda, m, \varphi_{j}
$$

with

$$
\delta \mathcal{Z}_{k, \chi}^{(X)}=\left.\left(t_{X}^{\varepsilon}\right)^{k} \delta \mathcal{Z}_{k, \chi}^{\left(X_{0}\right)}\right|_{\alpha=\alpha_{X}\left(\mu_{\mathrm{R}}^{2}\right)}
$$

In this way, all scale-dependent factors are reabsorbed into the coupling factors associated with the renormalisation constants.

The coupling constants corresponding to the schemes $X$ and $X_{0}$, defined through (4.18), (4.19) and (4.24)-(4.26), differ by a factor $t_{X}^{\varepsilon}=1+\mathcal{O}(\varepsilon)$. Thus these two schemes are not identical. Nevertheless they are equivalent since finite quantities in the scheme $X_{0}$ and $X$ differ only by irrelevant terms of $\mathcal{O}(\varepsilon)$. In particular, at the level of renormalised amplitudes the schemes $X$ and $X_{0}$ yield equivalent results. In the following sections we will treat scaledependent terms as in (4.24)-(4.26), which guarantees the consistency with the formalism of section 4.1 and makes it possible to use the renormalisation identities (4.8)-(4.16).

Note that the scale-independent parts $\delta \mathcal{Z}_{k, \chi}^{\left(X_{0}\right)}$ of (4.26) can involve terms of arbitrary high order in $\varepsilon$, and the $n$-loop renormalised amplitudes (4.13) receive finite contributions from all $\varepsilon$-suppressed terms of $\delta \mathcal{Z}_{k, \chi}^{\left(X_{0}\right)}$ up to order $\varepsilon^{n-k}$.

Minimal subtraction schemes. The MS and $\overline{\mathrm{MS}}$ schemes are simple realisations of the above scale-dependent renormalisation prescriptions. In the case of the MS scheme, the rescaling factor in (4.17) is simply

$$
S_{\mathrm{MS}}=1 .
$$

\footnotetext{
${ }^{5}$ To be more explicit, such finite renormalisation is defined through (4.21) in combination with

$$
\varphi_{j, X_{0}}=\varphi_{j, X}, \quad \theta_{i, X_{0}}\left(\mu_{\mathrm{R}}^{2}\right)=\theta_{i, X}\left(\mu_{\mathrm{R}}^{2}\right) \quad \text { for } \quad \theta_{i}=\lambda, m
$$
}

and the renormalisation constants in the schemes $X_{0}$ and $X$ are related via

$$
\mathcal{Z}_{\chi}^{(X)}=\left.\mathcal{Z}_{\chi}^{\left(X_{0}\right)}\right|_{\alpha=t_{X}^{\varepsilon} \alpha_{X}\left(\mu_{\mathrm{R}}^{2}\right)} \quad \text { for } \quad \chi=\alpha, \lambda, m, \varphi_{j}
$$


Thus for the rescaled renormalisation constants (4.26) we have

$$
\delta \mathcal{Z}_{k, \chi}^{(\mathrm{MS})}=\left(\frac{\mu_{0}^{2}}{\mu_{\mathrm{R}}^{2}}\right)^{k \varepsilon} \delta \mathcal{Z}_{k, \chi}^{\left(\mathrm{MS}_{0}\right)} \quad \text { for } \quad \chi=\alpha, \lambda, m, \varphi_{j},
$$

while their scale-independent parts involve only pure $1 / \varepsilon$ poles, i.e.

$$
\delta \mathcal{Z}_{k, \chi}^{\left(\mathrm{MS}_{0}\right)}=\left[\alpha_{\mathrm{MS}}\left(\mu_{\mathrm{R}}^{2}\right)\right]^{k} \sum_{m=1}^{k} \frac{b_{k, \chi}^{(m)}}{\varepsilon^{m}} .
$$

The difference between the MS and $\overline{\mathrm{MS}}$ schemes lies only in the rescaling parameter $S_{\overline{\mathrm{MS}}}$, which is defined through [37]

$$
\left(S_{\overline{\mathrm{MS}}}\right)^{\varepsilon}=(4 \pi)^{\varepsilon} \Gamma(1+\varepsilon)=\left(4 \pi e^{-\gamma_{\mathrm{E}}}\right)^{\varepsilon}+\mathcal{O}\left(\varepsilon^{2}\right) .
$$

Otherwise, the scale-independent parts of the renormalisation constants are equivalent. Thus

$$
\delta \mathcal{Z}_{k, \chi}^{(\overline{\mathrm{MS}})}=\left(\frac{S_{\overline{\mathrm{MS}}} \mu_{0}^{2}}{\mu_{\mathrm{R}}^{2}}\right)^{k \varepsilon} \delta \mathcal{Z}_{k, \chi}^{\left(\mathrm{MS}_{0}\right)} \quad \text { for } \quad \chi=\alpha, \lambda, m, \varphi_{j}
$$

Here it is implicitly understood that $\alpha_{\mathrm{MS}}\left(\mu_{\mathrm{R}}^{2}\right)$ is replaced by $\alpha_{\overline{\mathrm{MS}}}\left(\mu_{\mathrm{R}}^{2}\right)$ in $\delta \mathcal{Z}_{k, \chi}^{\left(\mathrm{MS}_{0}\right)}$.

For later convenience, we introduce also a generalised minimal subtraction scheme that we label $\mathrm{MS}_{X}$ and is defined through the renormalisation constants

$$
\delta \mathcal{Z}_{k, \chi}^{\left(\mathrm{MS}_{X}\right)}=\left(t_{X}^{\varepsilon}\right)^{k} \delta \mathcal{Z}_{k, \chi}^{\left(\mathrm{MS}_{0}\right)}=\left(\frac{S_{X} \mu_{0}^{2}}{\mu_{\mathrm{R}}^{2}}\right)^{k \varepsilon} \delta \mathcal{Z}_{k, \chi}^{\left(\mathrm{MS}_{0}\right)}
$$

where the rescaling factor $S_{X}$ is a freely adjustable parameter. The relation between the coupling constants in the schemes $\mathrm{MS}_{X}$ and MS can be easily derived from the scaleindependence of $\alpha_{0}$ and reads

$$
\alpha_{\mathrm{MS}}\left(\mu_{\mathrm{R}}^{2}\right)=\alpha_{\mathrm{MS}}\left(\mu_{\mathrm{R}}^{2} S_{X}^{-1}\right) .
$$

Let us analyse the scale dependence of renormalised amplitudes in the $\mathrm{MS}_{X}$ scheme,

$$
\mathbf{R}^{\left(\mathrm{MS}_{X}\right)} \overline{\mathcal{A}}_{n, \Gamma}\left(\mu_{\mathrm{R}}^{2}\right)=\sum_{m=0}^{n} D_{n-m}^{\left(\mathrm{MS}_{X}\right)} \overline{\mathcal{A}}_{m, \Gamma} .
$$

The r.h.s. depends on $\mu_{\mathrm{R}}$ and $S_{X}$ via the renormalisation operators

$$
D_{n-m}^{\left(\mathrm{MS}_{X}\right)}=\left(t_{X}^{\varepsilon}\right)^{n-m} D_{n-m}^{\left(\mathrm{MS}_{0}\right)} .
$$

Here the part $\left(t_{X}^{-\varepsilon}\right)^{m}$ of the scale-dependent factor can be reabsorbed into the unrenormalised amplitude $\overline{\mathcal{A}}_{m, \Gamma}$ by using

$$
\left(t_{X}^{-\varepsilon}\right)^{m} \overline{\mathcal{A}}_{m, \Gamma}=\left.\overline{\mathcal{A}}_{m, \Gamma}\right|_{\mu_{0}^{2}=\mu_{\mathrm{R}}^{2} S_{X}^{-1}},
$$


which follows from the fact that the dependence of $\overline{\mathcal{A}}_{m, \Gamma}$ on $\mu_{0}$ amounts to an overall factor $\left(\mu_{0}^{2 \varepsilon}\right)^{m}$ stemming from the integration measure (2.2). This yields

$$
\mathbf{R}^{\left(\mathrm{MS}_{X}\right)} \overline{\mathcal{A}}_{n, \Gamma}\left(\mu_{\mathrm{R}}^{2}\right)=\left.\left(t_{X}^{\varepsilon}\right)^{n} \sum_{m=0}^{n} D_{n-m}^{\left(\mathrm{MS}_{0}\right)} \overline{\mathcal{A}}_{m, \Gamma}\right|_{\mu_{0}^{2}=\mu_{\mathrm{R}}^{2} S_{X}^{-1}},
$$

where, due to the finiteness of the renormalised amplitude, the overall factor $\left(t_{X}^{\varepsilon}\right)^{n}$ generates only irrelevant $\mathcal{O}(\varepsilon)$ contributions, while the rest of the scale dependence is entirely controlled through the prescription $\mu_{0}^{2}=\mu_{\mathrm{R}}^{2} S_{X}^{-1}$ for unrenormalised amplitudes. For the special case of the MS scheme the above identity reads

$$
\mathbf{R}^{(\mathrm{MS})} \overline{\mathcal{A}}_{n, \Gamma}\left(\mu_{\mathrm{R}}^{2}\right)=\left.\left(t_{\mathrm{MS}}^{\varepsilon}\right)^{n} \sum_{m=0}^{n} D_{n-m}^{\left(\mathrm{MS}_{0}\right)} \overline{\mathcal{A}}_{m, \Gamma}\right|_{\mu_{0}^{2}=\mu_{\mathrm{R}}^{2}},
$$

where $t_{\mathrm{MS}}=\mu_{0}^{2} / \mu_{\mathrm{R}}^{2}$. Comparing the above equations we see that the renormalised amplitudes in the $\mathrm{MS}_{X}$ and MS schemes are connected through the same rescaling as in (4.33). More precisely,

$$
\mathbf{R}^{\left(\mathrm{MS}_{X}\right)} \overline{\mathcal{A}}_{n, \Gamma}\left(\mu_{\mathrm{R}}^{2}\right)=\left(S_{X}^{\varepsilon}\right)^{n} \mathbf{R}^{(\mathrm{MS})} \overline{\mathcal{A}}_{n, \Gamma}\left(\mu_{\mathrm{R}}^{2} S_{X}^{-1}\right)=\mathbf{R}^{(\mathrm{MS})} \overline{\mathcal{A}}_{n, \Gamma}\left(\mu_{\mathrm{R}}^{2} S_{X}^{-1}\right)+\mathcal{O}(\varepsilon) .
$$

A similar relation holds also for the dependence on the rescaling factor $S_{X}$ in a generic scheme $X$.

Scheme transformations. Let us now discuss generic transformations that connect two multiplicative renormalisation schemes of the form (4.24)-(4.26). Specifically we consider the transformation that connects the schemes $X$ and $\mathrm{MS}_{X}$ defined in (4.26) and (4.32). Such transformations can be formulated at the level of the renormalisation constants as

$$
\mathcal{Z}_{\chi}^{(X)}=\mathcal{Z}_{\chi}^{(\Delta X)} \mathcal{Z}_{\chi}^{\left(\mathrm{MS}_{X}\right)} \quad \text { for } \quad \chi=\alpha, \lambda, m, \varphi_{j}
$$

where the renormalisation constant

$$
\mathcal{Z}_{\chi}^{(\Delta X)}=1+\sum_{k=1}^{\infty} \delta \mathcal{Z}_{k, \chi}^{(\Delta X)}
$$

is free from $1 / \varepsilon$ poles. Its $k$-loop parts can be expressed as

$$
\mathcal{Z}_{k, \chi}^{(\Delta X)}=\left(t_{X}^{\varepsilon}\right)^{k} \mathcal{Z}_{k, \chi}^{\left(\Delta X_{0}\right)}
$$

where $\mathcal{Z}_{k, \chi}^{\left(\Delta X_{0}\right)}$ denotes the scale-independent part. Note that the scale factors $t_{X}^{\varepsilon}$ that enter the renormalisation constants $\mathcal{Z}_{\chi}^{(X)}, \mathcal{Z}_{\chi}^{(\Delta X)}$ and $\mathcal{Z}_{\chi}^{\left(\mathrm{MS}_{X}\right)}$ in (4.40) need to be identical. This is mandatory in order to match all logarithms of $t_{X}$ that arise from terms of type $\left(\alpha_{X} t_{X}^{\varepsilon}\right)^{n} \varepsilon^{-m}$.

Based on the factorisation of the renormalisation constants in (4.40), the renormalisation in the scheme $X$ can be regarded as a two-step procedure consisting of a subtraction of UV poles in the $\mathrm{MS}_{X}$ scheme and a subsequent multiplicative renormalisation with the 
finite renormalisation constants (4.41). More explicitly, the renormalised amplitudes (4.13) in the scheme $X$ can be obtained starting form $\mathrm{MS}_{X}$ renormalised amplitude through

$$
\mathbf{R}^{(X)} \overline{\mathcal{A}}_{\Gamma}\left(\left\{\theta_{i, X}\right\}\right)=\left[\prod_{j}\left(\mathcal{Z}_{\varphi_{j}}^{(\Delta X)}\right)^{1 / 2}\right] \mathbf{R}^{\left(\mathrm{MS}_{X}\right)} \overline{\mathcal{A}}_{\Gamma}\left(\left\{\theta_{i, \mathrm{MS}_{X}}\right\}\right),
$$

which is equivalent to

$$
\mathbf{R}^{(X)} \overline{\mathcal{A}}_{n, \Gamma}=\sum_{m=0}^{n} D_{n-m}^{(\Delta X)} \mathbf{R}^{\left(\mathrm{MS}_{X}\right)} \overline{\mathcal{A}}_{m, \Gamma},
$$

where it is understood that $\theta_{i}=\theta_{i, X}\left(\mu_{\mathrm{R}}^{2}\right)$ for all objects on the rhs, and the operators $D_{k}^{(\Delta X)}$ are defined as in (4.8)-(4.11) but with renormalisation constants $\delta \mathcal{Z}_{k}^{(X)}$ replaced by $\delta \mathcal{Z}_{k}^{(\Delta X)}$. Note that in (4.44) the parameter derivatives $\partial / \partial_{\theta_{i}}$ contained in $D_{k}^{(\Delta X)}$ act on all building blocks of $\mathbf{R}^{\left(\mathrm{MS}_{X}\right)} \overline{\mathcal{A}}_{m, \Gamma}$, i.e. both on amplitudes and renormalisation constants. At one and two loops (4.44) reads

$$
\begin{aligned}
& \mathbf{R}^{(X)} \overline{\mathcal{A}}_{1, \Gamma}=\mathbf{R}^{\left(\mathrm{MS}_{X}\right)} \overline{\mathcal{A}}_{1, \Gamma}+D_{1}^{(\Delta X)} \mathcal{A}_{0, \Gamma}, \\
& \mathbf{R}^{(X)} \overline{\mathcal{A}}_{2, \Gamma}=\mathbf{R}^{\left(\mathrm{MS}_{X}\right)} \overline{\mathcal{A}}_{2, \Gamma}+D_{1}^{(\Delta X)} \mathbf{R}^{(\mathrm{MS} X)} \overline{\mathcal{A}}_{1, \Gamma}+D_{2}^{(\Delta X)} \mathcal{A}_{0, \Gamma} .
\end{aligned}
$$

In the following sections, these identities will be used as a starting point to derive the master formulas (4.1) and (4.2) as well as the rules to transform rational terms from the minimal subtraction scheme to a generic scheme $X$. To this end, we will also make use of the relation

$$
D_{k}^{(X)}=\sum_{j=0}^{k} D_{k-j}^{(\Delta X)} D_{j}^{\left(\mathrm{MS}_{X}\right)},
$$

which can be derived by applying (4.13) on both sides of (4.44). At one and two loops, (4.47) reads

$$
\begin{aligned}
& D_{1}^{(X)}=D_{1}^{(\Delta X)}+D_{1}^{\left(\mathrm{MS}_{X}\right)} \\
& D_{2}^{(X)}=D_{2}^{(\Delta X)}+D_{1}^{(\Delta X)} D_{1}^{\left(\mathrm{MS}_{X}\right)}+D_{2}^{\left(\mathrm{MS}_{X}\right)} .
\end{aligned}
$$

\subsection{Renormalisation formulas in $D_{\mathrm{n}}=4$ in a generic scheme}

In this section we establish the generalised master formulas (4.1)-(4.2) by making use of finite multiplicative renormalisations. To this end, we first transform the original master formulas (2.7) and (3.12) to the $\mathrm{MS}_{X}$ scheme defined in (4.32), and we then extend them to the generic scheme $X$ by means of (4.45)-(4.46).

In [1] the analysis of rational terms was restricted to the MS and $\overline{\mathrm{MS}}$ schemes, and the regularisation scale $\mu_{0}$ has been identified with the renormalisation scale $\mu_{R}$. In order to generalise the results of [1] to the $\mathrm{MS}_{X}$ scheme with arbitrary scale $\mu_{0}$, let us start from the master formulas (2.7) and (3.12) in the MS scheme with $\mu_{0}=\mu_{\mathrm{R}}$,

$$
\begin{aligned}
\mathbf{R}^{(\mathrm{MS})} \overline{\mathcal{A}}_{1}= & \left.\mathcal{A}_{1}\right|_{\mu_{0}^{2}=\mu_{\mathrm{R}}^{2}}+\left(\delta Z_{1, \Gamma}^{\left(\mathrm{MS}_{0}\right)}+\delta \mathcal{R}_{1, \Gamma}^{\left(\mathrm{MS}_{0}\right)}\right) \\
\mathbf{R}^{(\mathrm{MS})} \overline{\mathcal{A}}_{2}= & \left.\mathcal{A}_{2}\right|_{\mu_{0}^{2}=\mu_{\mathrm{R}}^{2}}+\left.\sum_{\gamma}\left(\delta Z_{1, \gamma}^{(\mathrm{MS})}+\delta \tilde{Z}_{1, \gamma}^{\left(\mathrm{MS}_{0}\right)}+\delta \mathcal{R}_{1, \gamma}^{\left(\mathrm{MS}_{0}\right)}\right) \cdot \mathcal{A}_{1, \Gamma / \gamma}\right|_{\mu_{0}^{2}=\mu_{\mathrm{R}}^{2}} \\
& +\left(\delta Z_{2, \Gamma}^{\left(\mathrm{MS}_{0}\right)}+\delta \mathcal{R}_{2, \Gamma}^{\left(\mathrm{MS}_{0}\right)}\right) .
\end{aligned}
$$


Here all counterterms $\delta Z_{k}^{\left(\mathrm{MS}_{0}\right)}$ and $\delta \tilde{Z}_{k}^{\left(\mathrm{MS}_{0}\right)}$ involve only poles or order $\varepsilon^{-1}, \ldots, \varepsilon^{-k}$, and the rational counterterms $\delta \mathcal{R}_{k}^{\left(\mathrm{MS}_{0}\right)}$ involve only finite terms and poles of order $\varepsilon^{0}, \ldots, \varepsilon^{-k+1}$. In particular, for $\mu_{0}=\mu_{\mathrm{R}}$ all MS counterterms, including $\delta \mathcal{R}_{k}^{\left(\mathrm{MS}_{0}\right)}$, do not depend on any scale [1]. Note also that on the l.h.s. of (4.50)-(4.51) we do not indicate the special choice $\mu_{0}=\mu_{\mathrm{R}}$ since renormalised amplitudes are independent of $\mu_{0}$.

Based on (4.39) the above relations can be easily generalised to the $\mathrm{MS}_{X}$ scheme by setting $\mu_{0}^{2}=\mu_{\mathrm{R}}^{2} S_{X}^{-1}$ on the r.h.s. Moreover, along similar lines as in (4.34)-(4.37), the scale dependence can be reabsorbed into the counterterms by using

$$
\left.\mathcal{A}_{m}\right|_{\mu_{0}^{2}=\mu_{\mathrm{R}}^{2} S_{X}^{-1}}=\left(t_{X}^{-\varepsilon}\right)^{m} \mathcal{A}_{m},
$$

and multiplying $\mathbf{R}^{\left(\mathrm{MS}_{X}\right)} \overline{\mathcal{A}}_{n}$ by an overall factor $\left(t_{X}^{\varepsilon}\right)^{n}$. In this way, discarding irrelevant $\mathcal{O}(\varepsilon)$ terms, one arrives at

$$
\begin{aligned}
\mathbf{R}^{\left(\mathrm{MS}_{X}\right)} \overline{\mathcal{A}}_{1}= & \mathcal{A}_{1}+Z_{1, \Gamma}^{\left(\mathrm{MS}_{X}\right)}+\delta \mathcal{R}_{1, \Gamma}^{\left(\mathrm{MS}_{X}\right)} \\
\mathbf{R}^{\left(\mathrm{MS}_{X}\right)} \overline{\mathcal{A}}_{2}= & \mathcal{A}_{2}+\sum_{\gamma}\left(\delta Z_{1, \gamma}^{\left(\mathrm{MS}_{X}\right)}+\delta \tilde{Z}_{1, \gamma}^{\left(\mathrm{MS}_{X}\right)}+\delta \mathcal{R}_{1, \gamma}^{\left(\mathrm{MS}_{X}\right)}\right) \cdot \mathcal{A}_{1, \Gamma / \gamma} \\
& +\delta Z_{2, \Gamma}^{\left(\mathrm{MS}_{X}\right)}+\delta \mathcal{R}_{2, \Gamma}^{\left(\mathrm{MS}_{X}\right)}
\end{aligned}
$$

where all scale-dependent factors are absorbed into the counterterms and rational terms

$$
\delta Y_{k, \Gamma}^{\left(\mathrm{MS}_{X}\right)}=\left(t_{X}^{\varepsilon}\right)^{k} \delta Y_{k, \Gamma}^{\left(\mathrm{MS}_{0}\right)} \quad \text { for } \quad \delta Y=\delta Z, \delta \tilde{Z}, \delta \mathcal{R} .
$$

These relations can be directly converted to the MS or the $\overline{\mathrm{MS}}$ schemes by simply replacing $t_{X}^{\varepsilon}$ by $t_{\mathrm{MS}}^{\varepsilon}=\left(\mu_{0}^{2} / \mu_{\mathrm{R}}^{2}\right)^{\varepsilon}$ or $t_{\overline{\mathrm{MS}}}^{\varepsilon}=\left(S_{\overline{\mathrm{MS}}} \mu_{0}^{2} / \mu_{\mathrm{R}}^{2}\right)^{\varepsilon}$, respectively.

The explicit calculations of rational terms in [1] and in the present paper have been carried out in the $\overline{\mathrm{MS}}$ scheme using the special scale choice $\mu_{0}^{2}=\mu_{\mathrm{R}}^{2} S_{\overline{\mathrm{MS}}}^{-1}$, where $t_{\overline{\mathrm{MS}}}=1$, such that all counterterms are free from scale factors, and loop integrals are free from logarithms of $S_{\overline{\mathrm{MS}}}$.

In the following we derive the master formulas (4.1)-(4.2) as well as scheme transformation identities for the rational terms by combining (4.53)-(4.54) with the scheme transformations (4.45)-(4.46). In this context we use the trivial identity

$$
\delta Z_{k, \Gamma}^{(X)}=D_{k}^{(X)} \mathcal{A}_{0, \Gamma}
$$

which relates counterterms in the language of the $\mathbf{R}$-operation to the multiplicative renormalisation formalism.

The one-loop master formula (4.1) is obtained by applying (4.53) on the r.h.s. of (4.45). This results into

$$
\mathbf{R}^{(X)} \overline{\mathcal{A}}_{1, \Gamma}=\mathcal{A}_{1, \Gamma}+\left(\delta Z_{1, \Gamma}^{\left(\mathrm{MS}_{X}\right)}+\delta \mathcal{R}_{1, \Gamma}^{\left(\mathrm{MS}_{X}\right)}\right)+D_{1}^{(\Delta X)} \mathcal{A}_{0, \Gamma},
$$

which is equivalent to (4.1). Equating the two identities, and using (4.56) together with (4.48), one arrives at the following scheme-transformation formula for rational terms,

$$
\mathcal{R}_{1, \Gamma}^{(X)}=\mathcal{R}_{1, \Gamma}^{\left(\mathrm{MS}_{X}\right)}=t_{X}^{\varepsilon} \mathcal{R}_{1, \Gamma}^{\left(\mathrm{MS}_{0}\right)} .
$$


This means that, apart from the trivial $t_{X}^{\varepsilon}$ scale factor, one-loop rational terms are scheme independent. As we will see, this property holds also for the $\delta \tilde{Z}_{1}$ terms that appear on the r.h.s. of (4.2), i.e.

$$
\delta \tilde{Z}_{1, \Gamma}^{(X)}=\delta \tilde{Z}_{1, \Gamma}^{\left(\mathrm{MS}_{X}\right)}=t_{X}^{\varepsilon} \delta \tilde{Z}_{1, \Gamma}^{\left(\mathrm{MS}_{0}\right)}
$$

The two-loop master formula (4.2) can be derived by applying (4.53) and (4.54) on the r.h.s. of (4.46). This yields

$$
\begin{aligned}
\mathbf{R}^{(X)} \overline{\mathcal{A}}_{2, \Gamma}= & \mathcal{A}_{2, \Gamma}+\sum_{\gamma}\left(\delta Z_{1, \gamma}^{\left(\mathrm{MS}_{X}\right)}+\delta \tilde{Z}_{1, \gamma}^{\left(\mathrm{MS}_{X}\right)}+\delta \mathcal{R}_{1, \gamma}^{\left(\mathrm{MS}_{X}\right)}\right) \cdot \mathcal{A}_{1, \Gamma / \gamma}+\left(\delta Z_{2, \Gamma}^{\left(\mathrm{MS}_{X}\right)}+\delta \mathcal{R}_{2, \Gamma}^{\left(\mathrm{MS}_{X}\right)}\right) \\
& +D_{1}^{(\Delta X)}\left(\mathcal{A}_{1, \Gamma}+\delta Z_{1, \Gamma}^{\left(\mathrm{MS}_{X}\right)}+\delta \mathcal{R}_{1, \Gamma}^{\left(\mathrm{MS}_{X}\right)}\right)+D_{2}^{(\Delta X)} \mathcal{A}_{0, \Gamma}
\end{aligned}
$$

which is equivalent to (4.2). Equating the two relations, and using (4.56) with (4.49) and (4.58), yields (4.59) together with the following identity between rational terms in the $\mathrm{MS}_{X}$ and $X$ schemes,

$$
\begin{aligned}
\sum_{\gamma} \delta Z_{1, \gamma}^{(X)} \cdot \mathcal{A}_{1, \Gamma / \gamma}+\delta \mathcal{R}_{2, \Gamma}^{(X)}= & \sum_{\gamma} \delta Z_{1, \gamma}^{\left(\mathrm{MS}_{X}\right)} \cdot \mathcal{A}_{1, \Gamma / \gamma}+\delta \mathcal{R}_{2, \Gamma}^{\left(\mathrm{MS}_{X}\right)} \\
& +D_{1}^{(\Delta X)}\left(\mathcal{A}_{1, \Gamma}+\delta \mathcal{R}_{1, \Gamma}^{\left(\mathrm{MS}_{X}\right)}\right) .
\end{aligned}
$$

This scheme-transformation formula can be rewritten more compactly as

$$
\delta \mathcal{R}_{2, \Gamma}^{(X)}=\delta \mathcal{R}_{2, \Gamma}^{\left(\mathrm{MS}_{X}\right)}+D_{1}^{(\Delta X)} \delta \mathcal{R}_{1, \Gamma}^{\left(\mathrm{MS}_{X}\right)}+\delta \mathcal{K}_{2, \Gamma}^{(\Delta X)},
$$

with

$$
\delta \mathcal{K}_{2, \Gamma}^{(\Delta X)}=D_{1}^{(\Delta X)} \mathcal{A}_{1, \Gamma}-\sum_{\gamma} \delta Z_{1, \gamma}^{(\Delta X)} \cdot \mathcal{A}_{1, \Gamma / \gamma}
$$

The term $D_{1}^{(\Delta X)} \delta \mathcal{R}_{1, \Gamma}^{\left(\mathrm{MS}_{X}\right)}$ in (4.62) corresponds to the multiplicative renormalisation of the one-loop rational term, which requires, according to (4.10), the first derivative of $\delta \mathcal{R}_{1, \Gamma}^{\left(\mathrm{MS}_{X}\right)}$ with respect to all relevant parameters, including the gauge-fixing parameter $\lambda$. The remaining term (4.63) represents a nontrivial source of scheme dependence that originates from subtleties related to the subtraction of subdivergences in $D_{\mathrm{n}}=4$ dimensions. As discussed in detail in the next subsections, also this latter source of scheme dependence can be described in a general and process-independent way. In particular we will show that (4.63) can be expressed as a linear combination of one-loop renormalisation constants,

$$
\delta \mathcal{K}_{2, \Gamma}^{(\Delta X)}=\sum_{\chi} \delta \mathcal{Z}_{1, \chi}^{(\Delta X)} \delta \hat{\mathcal{K}}_{1, \Gamma}^{(\chi)}
$$

where $\delta \hat{\mathcal{K}}_{1, \Gamma}^{(\chi)}$ are scheme- and process-independent one-loop counterterms. Their explicit expressions are presented in section 4.5 . 


\subsection{Nontrivial scheme dependence of two-loop rational terms}

The origin of the scheme-dependent contribution (4.63) lies in the fact that the subtraction of UV subdivergences through multiplicative renormalisation in $D_{\mathrm{n}}=4$ dimensions yields different results depending on whether the renormalisation is carried out before projection to $D_{\mathrm{n}}=4$ dimensions, as defined in (4.3), or after.

The first term on the r.h.s. of (4.63) corresponds to the multiplicative renormalisation of a one-loop amplitude after projection to $D_{\mathrm{n}}=4$ dimensions. Schematically, regarding the integrand of the 1PI one-loop diagram $\Gamma$ as a product of internal propagators $G_{a}$ and vertices $\mathcal{V}_{b}$, one can write

$$
D_{1}^{(\Delta X)} \mathcal{A}_{1, \Gamma}=\sum_{G_{a}}\left[D_{1}^{(\Delta X)} G_{a}\right] \frac{\delta}{\delta G_{a}} \mathcal{A}_{1, \Gamma}+\sum_{\mathcal{V}_{b}}\left[D_{1}^{(\Delta X)} \mathcal{V}_{b}\right] \frac{\delta}{\delta \mathcal{V}_{b}} \mathcal{A}_{1, \Gamma}
$$

This schematic notation indicates that the loop propagators/vertices inside $\mathcal{A}_{1, \Gamma}$ are removed (one by one) by the derivative operators and replaced by the corresponding counterterms within square brackets. ${ }^{6}$ The sums on the r.h.s. run over all relevant types of propagators (fermions, gauge bosons, ghosts, scalars) and vertices. Similarly as for derivatives, the operator $D_{1}^{(\Delta X)}$ is linear, i.e. its effect on a set of diagrams amounts to the sum of the contributions of individual diagrams. For what concerns the renormalisation of parameters, i.e. the terms proportional to $\partial / \partial \theta_{i}$ in (4.10), the above identity corresponds to the chain rule. As for the renormalisation of fields, the overall effect amounts to a factor $\frac{1}{2} \delta \mathcal{Z}_{1, \varphi}^{(\Delta X)}$ for each external line, while all field-renormalisation factors associated with the internal loop lines cancel between vertices and propagators as usual.

The second term on the r.h.s. of (4.63) is defined through the prescription (4.3), where the renormalisation of $\overline{\mathcal{A}}_{1, \Gamma}$ is carried out before projecting to $D_{\mathrm{n}}=4$ dimensions. It can be expressed as follows in the from of one-loop insertions into the internal propagators $G_{a}$ and vertices $\mathcal{V}_{b}$ of $\mathcal{A}_{1, \Gamma}$,

$$
\sum_{\gamma} \delta Z_{1, \gamma}^{(\Delta X)} \cdot \mathcal{A}_{1, \Gamma / \gamma}=\sum_{G_{a}}\left[G_{a} \delta Z_{1, \Gamma a}^{(\Delta X)} G_{a}\right] \frac{\delta}{\delta G_{a}} \mathcal{A}_{1, \Gamma}+\sum_{\mathcal{V}_{b}} \delta Z_{1, \mathcal{V}_{b}}^{(\Delta X)} \frac{\delta}{\delta \mathcal{V}_{b}} \mathcal{A}_{1, \Gamma}
$$

The two types of counterterm on the r.h.s. correspond to the amputated 1PI two-point functions $\Gamma_{a}$, which are related to the propagators $G_{a}$ via (4.78) and (4.80), and to the amputated 1PI vertex functions $\mathcal{V}_{b}$. Such counterterms can be generated from the corre-

\footnotetext{
${ }^{6}$ More explicitly, let us consider a generic one-loop diagram $\Gamma$ containing $n_{a}$ propagators of type $a$, and let us denote as $k_{a}^{(j)}$ the loop momentum flowing through the $j^{\text {th }}$ type- $a$ propagator. In this case, the propagator-renormalisation operators on the r.h.s. of (4.65) should be understood as

$$
\left[D_{1}^{(\Delta X)} G_{a}\right] \frac{\delta}{\delta G_{a}} \mathcal{A}_{1, \Gamma}=\left.\sum_{j=1}^{n_{a}} \mathcal{A}_{1, \Gamma}\right|_{G_{a}\left(k_{a}^{j}, m_{a}\right) \rightarrow D_{1}^{(\Delta X)} G_{a}\left(k_{a}^{(j)}, m_{a}\right)}
$$

where the replacements are applied at the integrand level. The same holds also for vertex-renormalisation operators.
} 
sponding tree-level objects via multiplicative renormalisation, i.e.

$$
\sum_{\gamma} \delta Z_{1, \gamma}^{(\Delta X)} \cdot \mathcal{A}_{1, \Gamma / \gamma}=\sum_{G_{a}}\left[G_{a}\left(D_{1}^{(\Delta X)} \Gamma_{a}\right) G_{a}\right] \frac{\delta}{\delta G_{a}} \mathcal{A}_{1, \Gamma}+\sum_{\mathcal{V}_{b}}\left[D_{1}^{(\Delta X)} \mathcal{V}_{b}\right] \frac{\delta}{\delta \mathcal{V}_{b}} \mathcal{A}_{1, \Gamma}
$$

Note that in (4.65) and (4.67)-(4.68) all $G_{a}, \Gamma_{a}$ and $\mathcal{V}_{a}$ are in $D_{\mathrm{n}}=4$ dimensions, as indicated by the absence of a bar. As a consequence, in (4.67)-(4.68) all loop numerators are strictly four-dimensional. On the contrary, the term $D_{1}^{(\Delta X)} G_{a}$ in (4.65) can give rise to extra contributions proportional to $\tilde{q}^{2}$ in the loop numerator. This is due to the fact that the renormalisation of $G_{a}$ is carried out with $D$-dimensional denominator and fourdimensional numerator, i.e. after projection to $D_{\mathrm{n}}=4$. As we will see, the interplay of such $\tilde{q}^{2}$ terms with UV poles is at the origin of the auxiliary counterterm (4.63).

Comparing (4.65) to (4.68) we observe that (4.63) receives contributions only from the renormalisation of loop propagators and can be expressed as

$$
\delta \mathcal{K}_{2, \Gamma}^{(\Delta X)}=\sum_{G_{a}}\left(\mathcal{P}_{1}^{(\Delta X)} G_{a}\right) \frac{\delta}{\delta G_{a}} \mathcal{A}_{1, \Gamma}
$$

with

$$
\mathcal{P}_{1}^{(\Delta X)} G_{a}=\left[\left(D_{1}^{(\Delta X)} G_{a}\right)-G_{a}\left(D_{1}^{(\Delta X)} \Gamma_{a}\right) G_{a}\right] .
$$

Here the multiplicative renormalisation (4.10) of the 1PI two-point function $\Gamma_{a}$ yields

$$
D_{1}^{(\Delta X)} \Gamma_{a}=\left(\delta \mathcal{Z}_{1, \varphi_{a}}^{(\Delta X)}+\delta \mathcal{Z}_{1, m_{a}}^{(\Delta X)} m_{a} \partial_{m_{a}}+\delta \mathcal{Z}_{1, \lambda}^{(\Delta X)} \lambda \partial_{\lambda}\right) \Gamma_{a}
$$

where the renormalisation of the gauge parameter $\lambda$ is relevant only when $\Gamma_{a}$ is a gaugeboson two-point function. The first term on the r.h.s. of (4.70) corresponds to the renormalisation of a generic propagator and yields ${ }^{7}$

$$
D_{1}^{(\Delta X)} G_{a}=\left(-\delta \mathcal{Z}_{1, \varphi_{a}}^{(\Delta X)}+\delta \mathcal{Z}_{1, m_{a}}^{(\Delta X)} m_{a} \partial_{m_{a}}+\delta \mathcal{Z}_{1, \lambda}^{(\Delta X)} \lambda \partial_{\lambda}\right) G_{a}
$$

As discussed in the context of $(4.65)$, the factor $-\delta \mathcal{Z}_{1, \varphi_{a}}^{(\Delta X)}$ compensates the factors $\frac{1}{2} \delta \mathcal{Z}_{1, \varphi_{a}}^{(\Delta X)}$ associated with the vertices connected to the two ends of the propagator in such a way that, at the amplitude level, the net effect of field renormalisation amounts to a factor $\frac{1}{2} \delta \mathcal{Z}_{1, \varphi_{a}}^{(\Delta X)}$ per external leg.

Gauge-independent propagators. In the following we work out explicit expressions for the propagator corrections (4.70) starting from propagators that are gauge independent at tree level, i.e. the propagators of fermions, ghosts and physical scalar fields. In this case, with (4.71)-(4.72) we can express (4.70) as a linear combination of field and mass renormalisation constants,

$$
\mathcal{P}_{1}^{(\Delta X)} G_{a}=\left[\delta \mathcal{Z}_{1, \varphi_{a}}^{(\Delta X)} \hat{\mathcal{P}}_{1, \varphi}+\delta \mathcal{Z}_{1, m_{a}}^{(\Delta X)} \hat{\mathcal{P}}_{1, m}\right] G_{a},
$$

\footnotetext{
${ }^{7}$ Note that the sign of the field-renormalisation constants in (4.10) is meant for the renormalisation of amputated Green's functions, while the renormalisation of propagators requires the opposite sign.
} 
with scheme-independent operators

$$
\hat{\mathcal{P}}_{1, \varphi} G_{a}=-G_{a}-G_{a} \Gamma_{a} G_{a}
$$

and

$$
\hat{\mathcal{P}}_{1, m} G_{a}=m_{a} \partial_{m_{a}} G_{a}-G_{a}\left[m_{a} \partial_{m_{a}} \Gamma_{a}\right] G_{a} .
$$

In order to simplify the above identities, let us first discuss the general form of the tree-level propagator,

$$
G_{a} \equiv G_{a}\left(\bar{k}, m_{a}\right)=\frac{g_{a}\left(k, m_{a}\right)}{\bar{k}^{2}-m_{a}^{2}}
$$

where in $D_{\mathrm{n}}=4$ the numerator $g_{a}$ and the denominator are, respectively, in four and $D$ dimensions. This different dimensionality leads to a nontrivial relation between $g_{a}, G_{a}$ and $\Gamma_{a}$. The usual relation in $D_{\mathrm{n}}=D$ dimensions is

$$
\bar{g}_{a}\left(\bar{k}, m_{a}\right) \bar{\Gamma}_{a}\left(\bar{k}, m_{a}\right)=-\left(\bar{k}^{2}-m_{a}^{2}\right),
$$

or equivalently,

$$
\bar{G}_{a}\left(\bar{k}, m_{a}\right) \bar{\Gamma}_{a}\left(\bar{k}, m_{a}\right)=-1,
$$

i.e. $\bar{G}_{a}$ and $\bar{\Gamma}_{a}$ are the inverse of each other up to a minus sign. In contrast, for gaugeindependent propagators in $D_{\mathrm{n}}=4$ dimensions we have

$$
g_{a}\left(k, m_{a}\right) \Gamma_{a}\left(k, m_{a}\right)=-\left(k^{2}-m_{a}^{2}\right),
$$

and

$$
G_{a}\left(\bar{k}, m_{a}\right) \Gamma_{a}\left(k, m_{a}\right)=-\frac{k^{2}-m_{a}^{2}}{\bar{k}^{2}-m_{a}^{2}}=\frac{\tilde{q}^{2}}{\bar{k}^{2}-m_{a}^{2}}-1
$$

where $\bar{q}=q+\tilde{q}$ is the loop momentum, and $\bar{k}=\bar{q}+p$ is the momentum that flows through the loop propagator $G_{a}$. With this latter identity at hand we find that the fieldrenormalisation operator (4.74) corresponds to

$$
\hat{\mathcal{P}}_{1, \varphi} G_{a}\left(\bar{k}, m_{a}\right)=-G_{a}\left(\bar{k}, m_{a}\right) \frac{\tilde{k}^{2}}{\left(\bar{k}^{2}-m_{a}^{2}\right)} .
$$

As for the mass-renormalisation operator, the two terms on the r.h.s. of (4.75) can be simplified using

$$
\begin{aligned}
\left(\bar{k}^{2}-m_{a}^{2}\right) m_{a} \partial_{m_{a}} G_{a} & =m_{a} \partial_{m_{a}}\left[\left(\bar{k}^{2}-m_{a}^{2}\right) G_{a}\right]-G_{a} m_{a} \partial_{m_{a}}\left(\bar{k}^{2}-m_{a}^{2}\right) \\
& =m_{a} \partial_{m_{a}} g_{a}+2 m_{a}^{2} G_{a}
\end{aligned}
$$

and

$$
\begin{aligned}
\left(\bar{k}^{2}-m_{a}^{2}\right) G_{a}\left[m_{a} \partial_{m_{a}} \Gamma_{a}\right] G_{a} & =G_{a}\left[m_{a} \partial_{m_{a}} \Gamma_{a}\right] g_{a}=G_{a} m_{a} \partial_{m_{a}}\left(\Gamma_{a} g_{a}\right)-G_{a} \Gamma_{a} m_{a} \partial_{m_{a}} g_{a} \\
& =2 m_{a}^{2} G_{a}+\left(1-\frac{\tilde{q}^{2}}{\bar{k}^{2}-m_{a}^{2}}\right) m_{a} \partial_{m_{a}} g_{a}
\end{aligned}
$$


where we have exploited (4.76) and (4.79)-(4.80). Combining (4.75) with (4.82)-(4.83) one finds

$$
\hat{\mathcal{P}}_{1, m} G_{a}\left(\bar{k}, m_{a}\right)=\frac{\tilde{q}^{2}}{\left(\bar{k}^{2}-m_{a}^{2}\right)^{2}} m_{a} \partial_{m_{a}} g_{a} .
$$

In renormalisable gauge theories without symmetry breaking, the term $m_{a} \partial_{m_{a}} g_{a}$ is non-zero only for the propagators of massive fermions, for which $g_{a}\left(k, m_{a}\right)=\mathrm{i}\left(k+m_{a}\right)$. Thus

$$
\hat{\mathcal{P}}_{1, m} G_{a}\left(\bar{k}, m_{a}\right)=\frac{\tilde{q}^{2}}{\left(\bar{k}^{2}-m_{a}^{2}\right)^{2}} \times \begin{cases}\mathrm{i} m_{a} & \text { for fermion propagators } \\ 0 & \text { for ghost, scalar, and massless } \\ & \text { gauge-boson propagators. }\end{cases}
$$

Gauge-dependent propagators. Let us now consider the two-point function of massless gauge bosons in the so-called $\xi$-gauge. The relevant part of the renormalised Lagrangian reads

$$
\mathcal{L}_{\text {gauge }}=-\frac{1}{2}\left[\mathcal{Z}_{A} \partial_{\mu} A_{\nu}\left(\partial^{\mu} A^{\nu}-\partial^{\nu} A^{\mu}\right)+\frac{\mathcal{Z}_{A}}{\lambda \mathcal{Z}_{\lambda}}\left(\partial^{\mu} A_{\mu}\right)^{2}\right]
$$

Due to Ward identities, the renormalisation constants associated with the gauge field and the gauge-fixing parameter have identical UV poles. Thus it is convenient to define the latter as

$$
\mathcal{Z}_{\lambda}=\frac{\mathcal{Z}_{A}}{\mathcal{Z}_{\mathrm{gp}}}
$$

where $\mathcal{Z}_{\mathrm{gp}}$ is a finite renormalisation constant that is typically set equal to one.

At tree level in $D_{\mathrm{n}}=4$, the gauge-boson two-point function and the associated propagator read

$$
\Gamma_{A}^{\mu \nu}(k, \lambda)=-\mathrm{i}\left[k^{2} g^{\mu \nu}+\left(\frac{1}{\lambda}-1\right) k^{\nu} k^{\nu}\right]
$$

and

$$
G_{A}^{\mu \nu}(\bar{k}, \lambda)=-\frac{\mathrm{i}}{\bar{k}^{2}}\left[g^{\mu \nu}+(\lambda-1) \frac{k^{\nu} k^{\nu}}{\bar{k}^{2}}\right] .
$$

Since the two-point function is free from denominators, all objects on the r.h.s. of (4.88) are projected to four dimensions, while all denominators in (4.89) are in $D$ dimensions. Due to the different dimensionality of numerators and denominators, the transverse and longitudinal tensors in (4.89),

$$
P_{\mathrm{T}}^{\mu \nu}(\bar{k})=g^{\mu \nu}-\frac{k^{\nu} k^{\nu}}{\bar{k}^{2}}, \quad P_{\mathrm{L}}^{\mu \nu}(\bar{k})=\frac{k^{\nu} k^{\nu}}{\bar{k}^{2}},
$$

do not fulfil the usual projector properties. In particular,

$$
P_{\mathrm{T}}(\bar{k}) P_{\mathrm{L}}(\bar{k})=\frac{\tilde{k}^{2}}{\bar{k}^{2}} P_{\mathrm{L}}(\bar{k}) \neq 0
$$


As a consequence, instead of (4.80), for gauge-boson propagators we have

$$
G_{A \rho}^{\mu}(\bar{k}, \lambda) \Gamma_{A}^{\rho \nu}(\bar{k}, \lambda)=-g^{\mu \nu}+\frac{\tilde{k}^{2}}{\bar{k}^{2}}\left[g^{\mu \nu}+\left(1-\frac{1}{\lambda}\right) \frac{k^{\nu} k^{\nu}}{\bar{k}^{2}}\right] .
$$

Let us now work out the auxiliary counterterms (4.70) for gauge-bosons propagators. Along the same lines as in (4.73)-(4.75), we can express the operators (4.70)-(4.72) as linear combinations of the one-loop renormalisation constants $\delta Z_{1, A}$ and $\delta Z_{1, \mathrm{gp}}=\delta Z_{1, A}-\delta Z_{1, \lambda}$. This yields

$$
\begin{aligned}
D_{1}^{(\Delta X)} \Gamma_{A} & =\left[\delta \mathcal{Z}_{1, A}^{(\Delta X)}\left(1+\lambda \partial_{\lambda}\right)-\delta \mathcal{Z}_{1, \mathrm{gp}}^{(\Delta X)} \lambda \partial_{\lambda}\right] \Gamma_{A}, \\
D_{1}^{(\Delta X)} G_{A} & =\left[\delta \mathcal{Z}_{1, A}^{(\Delta X)}\left(-1+\lambda \partial_{\lambda}\right)-\delta \mathcal{Z}_{1, \mathrm{gp}}^{(\Delta X)} \lambda \partial_{\lambda}\right] G_{A},
\end{aligned}
$$

and

$$
\mathcal{P}_{1}^{(\Delta X)} G_{A}=\left[\delta \mathcal{Z}_{1, A}^{(\Delta X)} \hat{\mathcal{P}}_{1, A}+\delta \mathcal{Z}_{1, \mathrm{gp}}^{(\Delta X)} \hat{\mathcal{P}}_{1, \mathrm{gp}}\right] G_{a}
$$

with scheme-independent operators

$$
\hat{\mathcal{P}}_{1, A} G_{A}=\left(-1+\lambda \partial_{\lambda}\right) G_{A}-G_{A}\left[\left(1+\lambda \partial_{\lambda}\right) \Gamma_{A}\right] G_{A},
$$

and

$$
\hat{\mathcal{P}}_{1, \mathrm{gp}} G_{A}=-\lambda \partial_{\lambda} G_{A}+G_{A}\left[\lambda \partial_{\lambda} \Gamma_{A}\right] G_{A} .
$$

Inserting the explicit expressions (4.88)-(4.89) and using (4.92) we find

$$
\hat{\mathcal{P}}_{1, A} G_{A}^{\mu \nu}(\bar{k}, \lambda)=\frac{\tilde{k}^{2}}{\bar{k}^{2}}\left(\frac{\mathrm{i} g^{\mu \nu}}{\bar{k}^{2}}\right)
$$

and

$$
\hat{\mathcal{P}}_{1, \mathrm{gp}} G_{A}^{\nu \nu}(\bar{k}, \lambda)=\frac{\tilde{k}^{2}}{\bar{k}^{2}}(\lambda-1)\left[2+\left(\frac{1}{\lambda}-1\right) \frac{\tilde{k}^{2}}{\bar{k}^{2}}\right]\left(\frac{\mathrm{i} k^{\nu} k^{\nu}}{\bar{k}^{4}}\right) .
$$

Similarly as for the case of gauge-independent propagators, the above auxiliary counterterms are proportional to $\tilde{k}^{2} / \bar{k}^{2}$. Note also that the term (4.99) associated with the finite renormalisation of the gauge parameter vanishes in the Feynman gauge.

\subsection{Full scheme dependence of two-loop rational terms}

Combining the various results derived in section 4.4 one can express the nontrivial part of the two-loop scheme dependence (4.63) as a linear combination of field, mass and gaugeparameter renormalisation constants,

$$
\delta \mathcal{K}_{2, \Gamma}^{(\Delta X)}=\sum_{a}\left[\delta \mathcal{Z}_{1, \varphi_{a}}^{(\Delta X)} \delta \hat{\mathcal{K}}_{1, \Gamma}^{\left(\varphi_{a}\right)}+\delta \mathcal{Z}_{1, m_{a}}^{(\Delta X)} \delta \hat{\mathcal{K}}_{1, \Gamma}^{\left(m_{a}\right)}\right]+\delta \mathcal{Z}_{1, \mathrm{gp}}^{(\Delta X)} \delta \hat{\mathcal{K}}_{1, \Gamma}^{(\mathrm{gp})},
$$

where the sum extends over all kinds of fields $a$ (gauge bosons, fermions, ghosts or scalars) that propagate inside the loop diagrams contributing to the one-loop amplitude $\mathcal{A}_{1, \Gamma}$. The 
various coefficients $\delta \hat{\mathcal{K}}_{1, \Gamma}$ can be regarded as scheme-independent one-loop counterterms. The ones associated with the renormalisation of fields are given by

$$
\delta \hat{\mathcal{K}}_{1, \Gamma}^{\left(\varphi_{a}\right)}=\left(\hat{\mathcal{P}}_{1, \varphi} G_{a}\right) \frac{\delta}{\delta G_{a}} \mathcal{A}_{1, \Gamma}
$$

with

$$
\hat{\mathcal{P}}_{1, \varphi} G_{a}=\frac{\tilde{q}^{2}}{\left(\bar{k}^{2}-m_{a}^{2}\right)} \times \begin{cases}-G_{a}\left(\bar{k}, m_{a}\right) & \text { for fermion, ghost, and scalar propagators }, \\ \frac{\mathrm{i} g^{\mu \nu}}{\bar{k}^{2}} & \text { for massless gauge-boson propagators. }\end{cases}
$$

The counterterms associated with the mass renormalisation are given by

$$
\delta \hat{\mathcal{K}}_{1, \Gamma}^{\left(m_{a}\right)}=\left(\hat{\mathcal{P}}_{1, m} G_{a}\right) \frac{\delta}{\delta G_{a}} \mathcal{A}_{1, \Gamma},
$$

where $\hat{\mathcal{P}}_{1, m}$ is defined in (4.85). Finally, the counterterm associated with the finite renormalisation of the gauge parameter originates only from gauge-boson propagators $G_{A}$ and is given by

$$
\delta \hat{\mathcal{K}}_{1, \Gamma}^{(\mathrm{gp})}=\left(\hat{\mathcal{P}}_{1, \mathrm{gp}} G_{A}\right) \frac{\delta}{\delta G_{A}} \mathcal{A}_{1, \Gamma},
$$

where $\hat{\mathcal{P}}_{1, \mathrm{gp}}$ is defined in (4.99). For the standard choice $\mathcal{Z}_{\mathrm{gp}}=1$ this counterterm is irrelevant.

Note that all quantities in (4.100)-(4.104) are free from UV poles. In particular, the renormalisation constants $\delta \mathcal{Z}_{1, \chi}^{(\Delta X)}$ in (4.100) are UV finite. For this reason, the $\varepsilon$-expansion of the auxiliary counterterms (4.101)-(4.104) will be truncated by definition at order $\varepsilon^{0}$. With other words, terms of $\mathcal{O}(\varepsilon)$ in the above auxiliary counterterms will be discarded also when (4.100) is split into UV-divergent parts using

$$
\delta \mathcal{Z}_{1, \chi}^{(\Delta X)}=\delta \mathcal{Z}_{1, \chi}^{(X)}-\delta \mathcal{Z}_{1, \chi}^{\left(\mathrm{MS}_{X}\right)} .
$$

The counterterms (4.101)-(4.104) involve only finite parts that originate from the interplay of $\tilde{q}^{2}$ numerator terms with UV poles. Thus they are universal in the same sense as the usual renormalisation constants and rational terms.

From the viewpoint of UV power counting, the counterterms (4.101) and (4.104) correspond to the insertion of a term of order $\mathcal{O}\left(\tilde{q}^{2} / \bar{q}^{2}\right)$ into the original one-loop amplitude. Thus when $\mathcal{A}_{1, \Gamma}$ is UV divergent $\delta \hat{\mathcal{K}}_{1, \Gamma}^{\left(\varphi_{a}\right)}$ and $\delta \hat{\mathcal{K}}_{1, \Gamma}^{(\mathrm{gp})}$ are expected to be non-zero. In contrast, the counterterm (4.103) replaces a fermion propagators of $\mathcal{O}(1 / \bar{q})$ by objects of $\mathcal{O}\left(\tilde{q}^{2} / \bar{q}^{4}\right)$, thereby reducing the degree of UV divergence by one. Thus non-vanishing $\delta \hat{\mathcal{K}}_{1, \Gamma}^{\left(m_{a}\right)}$ contributions are expected only when $\mathcal{A}_{1, \Gamma}$ involves non-logarithmic UV divergences.

In summary, the identities (4.62) and (4.100)-(4.104) make it possible to transform twoloop rational terms from the minimal subtraction scheme (or any other reference scheme) to a generic renormalisation scheme $X$ using only universal one-loop quantities. Since all scheme-dependent parts in (4.62) are linear combinations of the finite renormalisation constants (4.105), the two-loop rational terms in the scheme $X$ can be expressed as

$$
\delta \mathcal{R}_{2, \Gamma}^{(X)}=\delta \mathcal{R}_{2, \Gamma}^{\left(\mathrm{MS}_{X}\right)}+\sum_{\chi} \delta \mathcal{Z}_{1, \chi}^{(\Delta X)} C_{1, \Gamma}^{(\chi)}
$$


where the sum over $\chi$ includes all relevant coupling-, gauge-, mass-, and field-renormalisation constants. Here the scheme dependence is isolated in the renormalisation constants $\delta \mathcal{Z}_{1, \chi}^{(\Delta X)}$, while their coefficients $C_{1, \Gamma}^{(\chi)}$, which are dictated by (4.62), are scheme independent. More precisely, their scheme dependence consist only of a trivial scale factor $t_{X}^{\varepsilon}$.

Contrary to what is suggested by the representation (4.106), the $\delta \mathcal{R}_{2, \Gamma}^{(X)}$ terms do not depend on the corresponding rational term in the $\mathrm{MS}_{X}$ scheme. This becomes evident by recasting (4.106), through (4.105), as a linear combination of the full renormalisation constants in the $X$ scheme,

$$
\delta \mathcal{R}_{2, \Gamma}^{(X)}=\delta \mathcal{R}_{2, \Gamma}^{(\mathrm{inv})}+\sum_{\chi} \delta \mathcal{Z}_{1, \chi}^{(X)} C_{1, \Gamma}^{(\chi)}
$$

In this representation $\delta \mathcal{R}_{2, \Gamma}^{\text {(inv) }}$ consists, apart from an overall scale factor $t_{X}^{2 \varepsilon}$, of terms of order $\varepsilon^{-1}$ and $\varepsilon^{0}$ that are independent of the schemes $X$ and $\mathrm{MS}_{X}$. In fact (4.107) and (4.106) imply that

$$
\delta \mathcal{R}_{2, \Gamma}^{(\mathrm{inv})}=\delta \mathcal{R}_{2, \Gamma}^{(X)}-\sum_{\chi} \delta \mathcal{Z}_{1, \chi}^{(X)} C_{1, \Gamma}^{(\chi)}=\delta \mathcal{R}_{2, \Gamma}^{\left(\mathrm{MS}_{X}\right)}-\sum_{\chi} \delta \mathcal{Z}_{1, \chi}^{\left(\mathrm{MS}_{X}\right)} C_{1, \Gamma}^{(\chi)},
$$

and, in practice, $\delta \mathcal{R}_{2, \Gamma}^{\text {(inv) }}$ can be derived from existing results in a minimal-subtraction scheme, or in any other scheme.

The general scheme-transformation properties derived in this section have been validated through a direct calculation of all QED and QCD two-loop rational terms in a generic scheme $X$.

\section{Two-loop rational counterterms for $\mathrm{SU}(\mathrm{N})$ and $\mathrm{U}(1)$ gauge theories}

In this section we present the full set of one- and two-loop rational counterterms for the generic Yang-Mills theory defined by the Lagrangian (5.2), which describes both $\mathrm{SU}(\mathrm{N})$ and $\mathrm{U}(1)$ gauge theories as special cases.

\subsection{Technical details of the calculations}

To compute all relevant $\delta \mathcal{R}_{1}$ and $\delta \mathcal{R}_{2}$ counterterms we have applied the master formula (3.19) to the full set of globally divergent Feynman diagrams that contribute to the various 1PI vertices with two, three and four external lines.

Based on the general scheme-transformation properties derived in section 4 , we have computed the $\delta \mathcal{R}_{2, \Gamma}$ counterterms in a generic renormalisation scheme. To this end we have recast the master formula (4.2) in the form (3.19). For the calculation of the relevant loop integrals we have employed the tadpole expansions presented in appendix A. The oneloop counterterms $\delta Z_{1, \gamma}, \delta \tilde{Z}_{1, \gamma}$ and $\delta \mathcal{R}_{1, \gamma}$ that are required for the derivation of two-loop rational counterterms can be found in section 5.3. In order to keep the scheme choice fully flexible we have decomposed all one-loop renormalisation constants as

$$
\delta \mathcal{Z}_{1, \chi}^{(X)}=\delta \mathcal{Z}_{1, \chi}^{\left(\mathrm{MS}_{X}\right)}+\delta \mathcal{Z}_{1, \chi}^{(\Delta X)}
$$


where the generalised minimal-subtraction constant $\delta \mathcal{Z}_{1, \chi}^{\left(\mathrm{MS}_{X}\right)}$, defined in (4.32), contains all explicit UV poles (see appendix B), while the finite remainder $\delta \mathcal{Z}_{1, \chi}^{(\Delta X)}$ is treated as a free parameter. The final results are presented in the form (4.107), i.e. as linear combinations of the full renormalisation constants $\delta \mathcal{Z}_{1, \chi}^{(X)}$. In the rest of this section the scheme label $X$ will be kept implicit.

All calculations have been performed twice and independently using different tools. On the one hand we have used GeFicom [38], which is based on QGraF [39], Q2E and ExP $[40,41]$ for the generation and topology identification of Feynman diagrams. Within GEFICOM algebraic manipulations, one-loop insertions and tadpole decompositions are implemented in Form [42, 43]. Massive tadpole integrals are computed with MATAD [44], and gauge-group factors with COLOR [45], both of which are based on FORM. Tadpole expansions are carried out with the methods described in appendices A.1 and A.4. The algebraic structures of the result are expressed in terms of a minimal set of independent Lorentz, Dirac and colour tensors, which are isolated in the beginning of the calculation using projectors that saturate all external indices.

To cross check all calculations we have developed a second in-house framework implemented in PYThon that uses QGRAF [39] for the amplitude generation and Form [42, 43] as well as PYTHON-FORM ${ }^{8}$ for the amplitude manipulations. In this framework all algebraic objects are directly reduced in the form of Dirac, Lorentz and group-theoretical tensors, i.e. without applying any projection to the indices associated with external lines. The gauge-group algebra is handled as described in section 5.2 or, alternatively, based on the colour-flow representation [46] for the $\mathrm{SU}(\mathrm{N})$ case. The tadpole expansions are implemented in the four different versions described in appendices A.1-A.4. The resulting tensorial tadpole integrals are expressed as combinations of metric tensors, and the coefficients are automatically reduced to master integrals with an in-house algorithm based on IBP identities [47, 48].

In both frameworks, two-loop amplitudes are directly decomposed into loop chains and connecting vertices according to (3.4) in such a way that enables the relevant powercounting operations and the further processing of the (sub-)diagrams. For what concerns dimensional regularisation, all calculations are carried out by handling $D=4-2 \varepsilon$ and the loop-numerator dimension $D_{\mathrm{n}}$ as independent free parameters. In this way all relevant UV poles and rational terms can be determined a posteriori by setting $D_{\mathrm{n}}=D$ and $D_{\mathrm{n}}=4$.

All results presented in section 5.3 have been derived independently in the two computing frameworks. In addition the following consistency checks have been carried out.

1. We have checked that all $\delta \mathcal{R}_{2}$ results are independent of the auxiliary mass $M$.

2. For all 1PI vertices in section 5.3 we have verified the cancellation of UV poles in the two-loop master formula (4.2). Note that the $\delta \mathcal{R}_{2}$ terms involve $1 / \varepsilon$ poles, and finite results are obtained only when all one- and two-loop counterterm contributions of $\mathrm{UV}$ and rational type are combined.

\footnotetext{
${ }^{8}$ https://github.com/tueda/python-form.
} 
3. To validate the consistency of the employed tadpole expansions, all calculations have been repeated using the four types of expansions presented in appendices A.1-A.4 finding consistent results. Note that changing the tadpole expansion method shifts the finite parts of the expanded amplitudes. Thus the validation at hand corresponds to a test of the master formula (4.2) at the level of the finite parts of the amplitudes.

4. We have checked that the Taylor-expansion method of appendix A.3 is independent of the choice of parametrisation. To this end we have carried out all $\delta \mathcal{R}_{2}$ calculations using independent parametrisations for one- and two-loop integrals.

5. We have verified that the renormalisation-scheme dependent parts of all $\delta \mathcal{R}_{2}$ counterterms are consistent with (4.62)-(4.63). To this end we have explicitly derived the $\delta \mathcal{K}_{2}$ parts using (4.100)-(4.104).

6. The one-loop counterterms $\delta Z_{1, \gamma}, \delta \tilde{Z}_{1, \gamma}$ and $\delta \mathcal{R}_{1, \gamma}$ that enter the calculation of $\delta \mathcal{R}_{2, \Gamma}$ have been treated in two alternative ways. On the one hand we have used available results at the level of full one-loop vertex functions $\gamma$. Alternatively, we have generated such counterterms at the level of individual two-loop diagrams by applying tadpole expansions to the relevant subdiagrams.

\subsection{Renormalised Lagrangian}

We have computed the rational counterterms for the generic Yang-Mills theory defined by the renormalised Lagrangian

$$
\mathcal{L}=\sum_{f \in \mathcal{F}} \mathcal{Z}_{f} \bar{\psi}_{f}\left(\mathrm{i} \gamma_{\mu} D^{\mu}-\mathcal{Z}_{m_{f}} m_{f}\right) \psi_{f}-\frac{1}{4} F_{\mu \nu}^{a} F^{a, \mu \nu}-\frac{\mathcal{Z}_{\mathrm{gp}}}{2 \lambda}\left(\partial^{\mu} A_{\mu}^{a}\right)^{2}-\mathcal{Z}_{u} \bar{u}^{a} \partial_{\mu} D_{a b}^{\mu} u^{b}
$$

with the field-strength tensor and the covariant derivatives

$$
\begin{aligned}
F_{\mu \nu}^{a} & =\mathcal{Z}_{A}^{1 / 2}\left[\partial_{\mu} A_{\nu}^{a}-\partial_{\nu} A_{\mu}^{a}+\left(\mathcal{Z}_{\alpha} \mathcal{Z}_{A}\right)^{1 / 2} g f^{a b c} A_{\mu}^{b} A_{\nu}^{c}\right] \\
D^{\mu} & =\partial^{\mu}-\mathrm{i}\left(\mathcal{Z}_{\alpha} \mathcal{Z}_{A}\right)^{1 / 2} g T^{a} A^{a \mu} \\
D_{a b}^{\mu} & =\partial^{\mu} \delta_{a b}-\left(\mathcal{Z}_{\alpha} \mathcal{Z}_{A}\right)^{1 / 2} g f^{a b c} A^{c \mu}
\end{aligned}
$$

where $t_{\mathrm{F}}^{a}=T^{a}$ and $\left(t_{\mathrm{A}}^{a}\right)_{b c}=-\mathrm{i} f^{a b c}$ are the generators of the gauge group in the fundamental and adjoint representations, while $g=\sqrt{4 \pi \alpha}$ is the gauge coupling. For the gauge fixing we adopt the Feynman gauge, which corresponds to $\lambda=1$. The gauge interaction acts on a certain number $n_{\mathrm{f}}$ of fermions, $f \in \mathcal{F}$, which belong to the fundamental representation, and the various fermion masses can have arbitrary values $m_{f} \geq 0$.

In the fundamental $(r=\mathrm{F})$ and adjoint $(r=\mathrm{A})$ representations, the generators satisfy the identities

$$
\left[t_{r}^{a}, t_{r}^{b}\right]=\mathrm{i} f^{a b c} t_{r}^{c}, \quad \operatorname{Tr}\left(t_{r}^{a} t_{r}^{b}\right)=T_{r} \delta^{a b},
$$

and the quadratic Casimir operators have eigenvalues

$$
C_{\mathrm{F}}=\frac{T_{\mathrm{F}} d_{\mathrm{A}}}{d_{\mathrm{F}}}, \quad C_{\mathrm{A}}=T_{\mathrm{A}},
$$




\begin{tabular}{c|cccccccc} 
& $d_{\mathrm{F}}$ & $T^{a}$ & $f^{a b c}$ & $\delta^{a b}$ & $d_{\mathrm{A}}$ & $T_{\mathrm{F}}$ & $C_{\mathrm{F}}$ & $C_{\mathrm{A}}$ \\
\hline $\mathrm{SU}(\mathrm{N})$ & $N$ & $T^{a}$ & $f^{a b c}$ & $\delta^{a b}$ & $N^{2}-1$ & $\frac{1}{2}$ & $\frac{N^{2}-1}{2 N}$ & $N$ \\
\hline $\mathrm{U}(1)$ & 1 & $Q_{f}$ & 0 & 1 & 1 & $Q_{f}^{2}$ & $Q_{f}^{2}$ & 0
\end{tabular}

Table 1. Values of the various group-theoretical quantities for $\mathrm{SU}(\mathrm{N})$ and $\mathrm{U}(1)$ gauge theories. In the $\mathrm{U}(1)$ case the replacements $T^{a} \rightarrow Q_{f}, T_{\mathrm{F}} \rightarrow Q_{f}^{2}$ and $C_{\mathrm{F}} \rightarrow Q_{f}^{2}$ involve the charge $Q_{f}$, where $f$ is the fermion on which $T^{a}, T_{\mathrm{F}}$ or $C_{\mathrm{F}}$ acts. For diagrams that involve external fermions one should use the substitution $C_{\mathrm{F}} \rightarrow Q_{f}^{2}$ at one loop, and the two-loop substitutions $C_{\mathrm{F}}^{2} \rightarrow Q_{f}^{4}$ and $n_{\mathrm{f}} T_{\mathrm{F}} C_{\mathrm{F}} \rightarrow Q_{f}^{2} \sum_{f^{\prime}} Q_{f^{\prime}}^{2}$, where the sum runs over all fermions $f^{\prime} \in \mathcal{F}$ that circulate in closed loops. Instead, for vertices without external fermions one should use the one-loop substitutions $n_{\mathrm{f}} T_{\mathrm{F}} \rightarrow$ $\sum_{f^{\prime}} Q_{f^{\prime}}^{2}$ and $T_{\mathrm{F}} \sum_{f^{\prime}} m_{f^{\prime}}^{2} \rightarrow \sum_{f^{\prime}} Q_{f^{\prime}}^{2} m_{f^{\prime}}^{2}$, and the two-loop substitutions $n_{\mathrm{f}} T_{\mathrm{F}} C_{\mathrm{F}} \rightarrow \sum_{f^{\prime}} Q_{f^{\prime}}^{4}$ and $T_{\mathrm{F}} \sum_{f^{\prime}} C_{\mathrm{F}} m_{f^{\prime}}^{2} \rightarrow \sum_{f^{\prime}} Q_{f^{\prime}}^{4} m_{f^{\prime}}^{2}$.

where $d_{r}$ denotes the dimension of the $r$ representation, and $d_{\mathrm{F}}=N$. Our results are expressed in terms of the invariants $C_{\mathrm{F}}, C_{\mathrm{A}}, N$ and $T_{\mathrm{F}}$. Note that the normalisation of all generators and combinations thereof is controlled by $T_{\mathrm{F}}$. In particular, $T^{a}$ and $f^{a b c}$ scale like $T_{\mathrm{F}}^{1 / 2}$, while $C_{\mathrm{F}}$ and $C_{\mathrm{A}}$ scale like $T_{\mathrm{F}}$.

For all two- and three-point counterterms presented in section 5.3 we have obtained compact expressions using generic identities that are valid for any simple or abelian gauge group, while for the four-point counterterm (5.30) we have employed identities like

$$
T_{i j}^{a} T_{k l}^{a}=C_{\mathrm{A}} T_{\mathrm{F}}\left(\frac{1}{N} \delta_{i l} \delta_{k j}-\delta_{i j} \delta_{k l}\right)+C_{\mathrm{F}} \delta_{i j} \delta_{k l}
$$

which are valid for $\mathrm{SU}(\mathrm{N})$ and $\mathrm{U}(1)$ groups. The explicit expressions of the rational counterterms for $\mathrm{SU}(\mathrm{N})$ and $\mathrm{U}(1)$ gauge theories can be obtained from the results of section 5.3 by applying the substitutions listed in table 5.2. The $\mathrm{SU}(\mathrm{N})$ case with $N=3$ and $\alpha=\alpha_{\mathrm{S}}$ corresponds to QCD with $n_{\mathrm{f}}$ active quarks with masses $m_{f} \geq 0$, while the $\mathrm{U}(1)$ case with $\alpha=\alpha_{\mathrm{EM}}$ corresponds to QED with $n_{\mathrm{f}}$ fermions with charges $Q_{f}$ and masses $m_{f} \geq 0$.

The renormalisation scheme is specified through generic renormalisation constants using the formalism of section 4 . The constants $\mathcal{Z}_{\alpha}$ and $\mathcal{Z}_{m_{f}}$ renormalise $\alpha$ and the fermion masses, while $\mathcal{Z}_{\mathrm{gp}}=\mathcal{Z}_{A} / \mathcal{Z}_{\lambda}$ is a finite parameter that renormalises the gauge-fixing term, see (4.87). Finally, the constants $\mathcal{Z}_{f}, \mathcal{Z}_{A}$ and $\mathcal{Z}_{u}$ control the renormalisation of the fermion, gauge-boson and ghost fields. At the level of renormalised amplitudes, the net effect of field renormalisation amounts to a factor $\left(\mathcal{Z}_{\varphi_{\text {ext }}}\right)^{1 / 2}$ for each external leg associated with the field $\varphi_{\text {ext }}$. Note, however, that the $\delta \mathcal{R}_{2, \Gamma}$ counterterms depend also on other fieldrenormalisation constants. This dependence originates from the contributions $\delta \mathcal{K}_{2, \Gamma}^{(\Delta X)}$, which are defined in (4.62)-(4.63) and depend, see (4.100), on the $\delta \mathcal{Z}_{1, \varphi_{\text {in }}}$ factors associated with the renormalisation of one-loop $\varphi_{\text {in }}$-selfenergy subdiagrams. In the renormalised two-loop amplitude (4.2) the dependence on $\delta \mathcal{Z}_{1, \varphi_{\text {in }}}$ cancels when the $\delta \mathcal{R}_{2, \Gamma}$ counterterm is combined with the contribution of the $\delta Z_{1, \gamma}$ counterterms associated with $\varphi_{\text {in }}$ selfenergies. This nontrivial cancellation mechanism can be exploited to validate the implementation of the master formula (4.2). 
The perturbative expansion of the various renormalisation constants is written in the form

$$
\mathcal{Z}_{\chi}=1+\sum_{k=1}^{\infty}\left(\frac{\alpha t^{\varepsilon}}{4 \pi}\right)^{k} \delta \hat{\mathcal{Z}}_{k, \chi} \quad \text { for } \quad \chi=\alpha, m_{f}, f, A, u, \mathrm{gp},
$$

where $t=S \mu_{0}^{2} / \mu_{\mathrm{R}}^{2}$ embodies the dependence on the regularisation scale $\mu_{0}$, the renormalisation scale $\mu_{\mathrm{R}}$, and the rescaling factor $S$ (see section 4.2). At variance with [1], where $\mu_{0}$ was set equal to $\mu_{\mathrm{R}}$, here $\mu_{0}, \mu_{\mathrm{R}}$ and $S$ are treated as independent parameters. Note that the renormalisation-scheme label $X$ used in section 4 is kept implicit in this section. Still, the renormalisation constants (5.7) describe a fully generic renormalisation scheme, which may be the minimal subtraction scheme, the on-shell scheme, or any other scheme. It is implicitly understood that the renormalised parameters depend on the renormalisation scale $\mu_{\mathrm{R}}$, but, depending on the scheme, $\mu_{\mathrm{R}}$ may be replaced by a physical mass scale, such as $m_{e}$ or $M_{Z}$. Explicit expressions for the various renormalisation constants in the $\overline{\mathrm{MS}}$ scheme are reported in appendix B.

\subsection{Rational counterterms}

In the following we present the rational and UV counterterms for the Yang-Mills Lagrangian (5.2) at order $\alpha$ and $\alpha^{2}$. As usual UV singularities are regularised in $D=4-2 \varepsilon$ dimensions. The rational terms associated with a certain 1PI vertex function $\Gamma$ are presented in the form

$$
\delta \mathcal{R}_{k, \Gamma}^{\alpha_{1} \ldots \alpha_{N}}=\mathrm{i}\left(\frac{\alpha t^{\varepsilon}}{4 \pi}\right)^{k} \sum_{a} \delta \hat{\mathcal{R}}_{k, \Gamma}^{(a)} \mathcal{T}_{a, \Gamma}^{\alpha_{1} \ldots \alpha_{N}}
$$

where $k=1,2$ is the loop order, and $\mathcal{T}_{a \Gamma \Gamma}^{\alpha_{1} \ldots \alpha_{N}}$ are independent tensor structures carrying the indices $\alpha_{1} \ldots \alpha_{N}$ of the external lines of the vertex function at hand. A similar decomposition is used also for the full $k$-loop counterterm $\delta Z_{k, \Gamma}^{\alpha_{1} \ldots \alpha_{N}}$ associated with $\Gamma$.

We recall that, when one-loop counterterms $\delta Z_{1, \gamma}^{\alpha_{1} \ldots \alpha_{N}}\left(q_{1}\right)$ are inserted into one-loop diagrams in the context of two-loop calculations, the associated tensor structures and their dependence on the loop momentum $q_{1}$ have to be adapted to the dimensionality of the loop numerator, i.e. in $D_{\mathrm{n}}=D$ and $D_{\mathrm{n}}=4$ numerator dimensions $\delta Z_{1, \gamma}^{\bar{\alpha}_{1} \ldots \bar{\alpha}_{N}}\left(\bar{q}_{1}\right)$ and $\delta Z_{1, \gamma}^{\alpha_{1} \ldots \alpha_{N}}\left(q_{1}\right)$ have to be used, respectively.

Fermion two-point function. For the two-point function of a fermion $f$ with mass $m_{f}$ we have

$$
\begin{aligned}
& \stackrel{i_{1}, \alpha_{1}}{\longleftarrow}-\stackrel{i_{2}, \alpha_{2}}{\longleftarrow}=\mathrm{i} \delta_{i_{1} i_{2}}\left\{\left(\not p-m_{f}\right)_{\alpha_{1} \alpha_{2}}\right. \\
& \left.\quad+\sum_{k=1}^{2}\left(\frac{\alpha t^{\varepsilon}}{4 \pi}\right)^{k}\left[\left(\delta \hat{Z}_{k, \mathrm{ff}}^{(\mathrm{P})}+\delta \hat{\mathcal{R}}_{k, \mathrm{ff}}^{(\mathrm{P})}\right) \not \phi_{\alpha_{1} \alpha_{2}}+\left(\delta \hat{Z}_{k, \mathrm{ff}}^{(\mathrm{m})}+\delta \hat{\mathcal{R}}_{k, \mathrm{ff}}^{(\mathrm{m})}\right) m_{f} \delta_{\alpha_{1} \alpha_{2}}\right]\right\}
\end{aligned}
$$

with UV counterterms

$$
\begin{array}{ll}
\delta \hat{Z}_{1, \mathrm{ff}}^{(\mathrm{P})}=\delta \hat{\mathcal{Z}}_{1, f}, & \delta \hat{Z}_{2, \mathrm{ff}}^{(\mathrm{P})}=\delta \hat{\mathcal{Z}}_{2, f}, \\
\delta \hat{Z}_{1, \mathrm{ff}}^{(\mathrm{m})}=-\delta \hat{\mathcal{Z}}_{1, f}-\delta \hat{\mathcal{Z}}_{1, m_{f}}, & \delta \hat{Z}_{2, \mathrm{ff}}^{(\mathrm{m})}=-\delta \hat{\mathcal{Z}}_{2, f}-\delta \hat{\mathcal{Z}}_{2, m_{f}}-\delta \hat{\mathcal{Z}}_{1, f} \delta \hat{\mathcal{Z}}_{1, m_{f}},
\end{array}
$$


and rational counterterms

$$
\begin{aligned}
\delta \hat{\mathcal{R}}_{1, \mathrm{ff}}^{(\mathrm{P})}= & -C_{\mathrm{F}} \\
\delta \hat{\mathcal{R}}_{2, \mathrm{ff}}^{(\mathrm{P})}= & \left(\frac{7}{6} C_{\mathrm{F}}^{2}-\frac{61}{36} C_{\mathrm{A}} C_{\mathrm{F}}+\frac{5}{9} T_{\mathrm{F}} n_{\mathrm{f}} C_{\mathrm{F}}\right) \varepsilon^{-1}+\frac{43}{36} C_{\mathrm{F}}^{2}-\frac{1087}{216} C_{\mathrm{A}} C_{\mathrm{F}}+\frac{59}{54} T_{\mathrm{F}} n_{\mathrm{f}} C_{\mathrm{F}} \\
& -C_{\mathrm{F}}\left(\delta \hat{\mathcal{Z}}_{1, \alpha}+\frac{2}{3} \delta \hat{\mathcal{Z}}_{1, f}-\frac{2}{3} \delta \hat{\mathcal{Z}}_{1, \mathrm{gp}}\right) \\
\delta \hat{\mathcal{R}}_{1, \mathrm{ff}}^{(\mathrm{m})}= & 2 C_{\mathrm{F}} \\
\delta \hat{\mathcal{R}}_{2, \mathrm{ff}}^{(\mathrm{m})}= & \left(-2 C_{\mathrm{F}}^{2}+\frac{61}{12} C_{\mathrm{A}} C_{\mathrm{F}}-\frac{5}{3} T_{\mathrm{F}} n_{\mathrm{f}} C_{\mathrm{F}}\right) \varepsilon^{-1}+C_{\mathrm{F}}^{2}+\frac{199}{24} C_{\mathrm{A}} C_{\mathrm{F}}-\frac{11}{6} T_{\mathrm{F}} n_{\mathrm{f}} C_{\mathrm{F}} \\
& +C_{\mathrm{F}}\left(2 \delta \hat{\mathcal{Z}}_{1, \alpha}+4 \delta \hat{\mathcal{Z}}_{1, m_{f}}-\frac{3}{2} \delta \hat{\mathcal{Z}}_{1, A}-\frac{1}{2} \delta \hat{\mathcal{Z}}_{1, \mathrm{gp}}\right)
\end{aligned}
$$

As usual the direction of the momentum $p$ in (5.9) coincides with the fermion flow.

Gauge-boson two-point function. For the gauge-boson two-point function we have

$$
\begin{aligned}
& {\underset{\mu}{a_{1}}}_{\mu_{1}}^{a_{2}}=\mathrm{i} \delta^{a_{1} a_{2}}\left\{-p^{2} g^{\mu_{1} \mu_{2}}\right. \\
& \left.+\sum_{k=1}^{2}\left(\frac{\alpha t^{\varepsilon}}{4 \pi}\right)^{k}\left[\left(\delta \hat{Z}_{k, \mathrm{gg}}^{(\mathrm{P})}+\delta \hat{\mathcal{R}}_{k, \mathrm{gg}}^{(\mathrm{P})}\right) p^{\mu_{1}} p^{\mu_{2}}+\left(\delta \hat{Z}_{k, \mathrm{gg}}^{(\mathrm{G})} p^{2}+\delta \hat{\mathcal{R}}_{k, \mathrm{gg}}^{(\mathrm{G})} p^{2}+\delta \tilde{Z}_{k, \mathrm{gg}}^{(\mathrm{G})} \tilde{p}^{2}\right) g^{\mu_{1} \mu_{2}}\right]\right\},
\end{aligned}
$$

with UV counterterms

$$
\begin{array}{ll}
\delta \hat{Z}_{1, \mathrm{gg}}^{(\mathrm{P})}=\delta \hat{\mathcal{Z}}_{1, A}-\delta \hat{\mathcal{Z}}_{1, \mathrm{gp}}, & \delta \hat{Z}_{2, \mathrm{gg}}^{(\mathrm{P})}=\delta \hat{\mathcal{Z}}_{2, A}-\delta \hat{\mathcal{Z}}_{2, \mathrm{gp}}, \\
\delta \hat{Z}_{1, \mathrm{gg}}^{(\mathrm{G})}=-\delta \hat{\mathcal{Z}}_{1, A}, & \delta \hat{Z}_{2, \mathrm{gg}}^{(\mathrm{G})}=-\delta \hat{\mathcal{Z}}_{2, A},
\end{array}
$$

and rational counterterms

$$
\begin{aligned}
\delta \hat{\mathcal{R}}_{1, \mathrm{gg}}^{(\mathrm{P})}= & -\frac{C_{\mathrm{A}}}{3}, \\
\delta \hat{\mathcal{R}}_{2, \mathrm{gg}}^{(\mathrm{P})}= & {\left[\frac{19}{36} C_{\mathrm{A}}^{2}+T_{\mathrm{F}} n_{\mathrm{f}}\left(-\frac{32}{9} C_{\mathrm{A}}+2 C_{\mathrm{F}}\right)\right] \varepsilon^{-1}+T_{\mathrm{F}} n_{\mathrm{f}}\left(\frac{217}{108} C_{\mathrm{A}}-\frac{71}{18} C_{\mathrm{F}}\right) } \\
& +\frac{1211}{864} C_{\mathrm{A}}^{2}+C_{\mathrm{A}}\left(-\frac{1}{3} \delta \hat{\mathcal{Z}}_{1, \alpha}-\frac{35}{12} \delta \hat{\mathcal{Z}}_{1, A}+\frac{3}{4} \delta \hat{\mathcal{Z}}_{1, \mathrm{gp}}+\frac{1}{6} \delta \hat{\mathcal{Z}}_{1, u}\right) \\
& +\frac{4}{3} T_{\mathrm{F}} \sum_{f \in \mathcal{F}} \delta \hat{\mathcal{Z}}_{1, f}
\end{aligned}
$$


and

$$
\begin{aligned}
\delta \hat{\mathcal{R}}_{1, \mathrm{gg}}^{(\mathrm{G})}= & \left(\frac{C_{\mathrm{A}}}{2}+\frac{2}{3} T_{\mathrm{F}} n_{\mathrm{f}}\right)-4 T_{\mathrm{F}} \sum_{f \in \mathcal{F}} \frac{m_{f}^{2}}{p^{2}}, \\
\delta \hat{\mathcal{R}}_{2, \mathrm{gg}}^{(\mathrm{G})}= & {\left[-\frac{4}{9} C_{\mathrm{A}}^{2}+T_{\mathrm{F}} n_{\mathrm{f}}\left(\frac{35}{9} C_{\mathrm{A}}-2 C_{\mathrm{F}}\right)\right] \varepsilon^{-1}+T_{\mathrm{F}} n_{\mathrm{f}}\left(-\frac{193}{108} C_{\mathrm{A}}+\frac{109}{36} C_{\mathrm{F}}\right) } \\
& -\frac{541}{432} C_{\mathrm{A}}^{2}-T_{\mathrm{F}} \sum_{f \in \mathcal{F}}\left[\left(C_{\mathrm{A}}+6 C_{\mathrm{F}}\right) \varepsilon^{-1}+\frac{13}{6} C_{\mathrm{A}}-7 C_{\mathrm{F}}\right] \frac{m_{f}^{2}}{p^{2}} \\
& +\left(\frac{C_{\mathrm{A}}}{2}+\frac{2}{3} T_{\mathrm{F}} n_{\mathrm{f}}\right) \delta \hat{\mathcal{Z}}_{1, \alpha}+\left(\frac{71}{24} C_{\mathrm{A}}+\frac{2}{3} T_{\mathrm{F}} n_{\mathrm{f}}\right) \delta \hat{\mathcal{Z}}_{1, A}-\frac{7}{8} C_{\mathrm{A}} \delta \hat{\mathcal{Z}}_{1, \mathrm{gp}} \\
& +\frac{C_{\mathrm{A}}}{12} \delta \hat{\mathcal{Z}}_{1, u}-4 T_{\mathrm{F}} \sum_{f \in \mathcal{F}}\left[\frac{1}{3} \delta \hat{\mathcal{Z}}_{1, f}+\left(\delta \hat{\mathcal{Z}}_{1, \alpha}+\delta \hat{\mathcal{Z}}_{1, A}+\delta \hat{\mathcal{Z}}_{1, m_{f}}\right) \frac{m_{f}^{2}}{p^{2}}\right] .
\end{aligned}
$$

In addition, due to the presence of a quadratic divergence, the usual UV counterterm for the gluon two-point function needs to be supplemented by

$$
\delta \tilde{Z}_{1, \mathrm{gg}}^{(\mathrm{G})}=\left(\frac{2}{3} C_{\mathrm{A}}+\frac{2}{3} T_{\mathrm{F}} n_{\mathrm{f}}\right) \varepsilon^{-1} .
$$

This extra term is relevant only when it is inserted into a one-loop diagram in the context of two-loop calculations, and its two-loop extension $\delta \tilde{Z}_{2 \text {,gg }}^{(\mathrm{G})}$ is required only for calculations beyond two loops.

Ghost two-point function. For the ghost two-point function we have

$$
\stackrel{a_{1}}{a_{-} \rightarrow--}=\mathrm{i} \delta^{a_{1} a_{2}}\left\{p^{2}+\sum_{k=1}^{2}\left(\frac{\alpha t^{\varepsilon}}{4 \pi}\right)^{k}\left(\delta \hat{Z}_{k, \mathrm{uu}}^{(\mathrm{P})}+\delta \hat{\mathcal{R}}_{k, \mathrm{uu}}^{(\mathrm{P})}\right) p^{2}\right\},
$$

with UV counterterms

$$
\delta \hat{Z}_{1, \mathrm{uu}}^{(\mathrm{P})}=\delta \hat{\mathcal{Z}}_{1, u}, \quad \delta \hat{Z}_{2, \mathrm{uu}}^{(\mathrm{P})}=\delta \hat{\mathcal{Z}}_{2, u},
$$

and rational counterterms

$$
\begin{aligned}
\delta \hat{\mathcal{R}}_{1, \mathrm{uu}}^{(\mathrm{P})}= & 0 \\
\delta \hat{\mathcal{R}}_{2, \mathrm{uu}}^{(\mathrm{P})}= & \left(\frac{7}{18} C_{\mathrm{A}}^{2}-\frac{5}{18} T_{\mathrm{F}} n_{\mathrm{f}} C_{\mathrm{A}}\right) \varepsilon^{-1}+\frac{10}{27} C_{\mathrm{A}}^{2}-\frac{17}{108} T_{\mathrm{F}} n_{\mathrm{f}} C_{\mathrm{A}} \\
& -C_{\mathrm{A}}\left(\frac{1}{2} \delta \hat{\mathcal{Z}}_{1, A}-\frac{1}{6} \delta \hat{\mathcal{Z}}_{1, \mathrm{gp}}+\frac{1}{6} \delta \hat{\mathcal{Z}}_{1, u}\right) .
\end{aligned}
$$

The vanishing of the one-loop rational term is due to the fact that, apart from coupling factors, the numerator of the ghost one-loop selfenergy is simply given by $\bar{q}_{\bar{\mu}} p^{\mu}=q_{\mu} p^{\mu}$, where $p$ is the external momentum, and is thus free form $(D-4)$-dimensional parts. Note also that the quadratic mass dimension of the ghost two-point function may require a $\tilde{p}^{2} / \varepsilon$ counterterm of type (2.13). However, this is not the case since, due to the absence of quadratic terms in $q$ in the loop numerator, the one-loop ghost selfenergy is free from quadratic divergences. 
Gauge-boson-fermion three-point vertex. For the gauge-boson-fermion-antifermion vertex we have

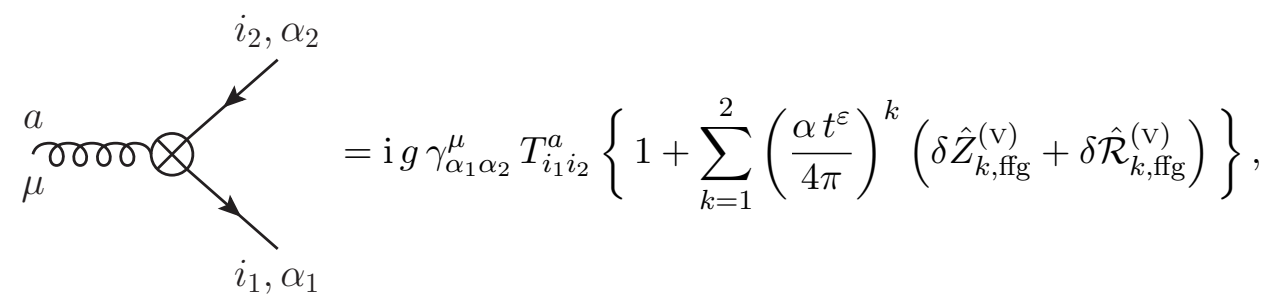

with UV counterterms

$$
\begin{aligned}
\delta \hat{Z}_{1, \mathrm{ffg}}^{(\mathrm{v})}= & \frac{1}{2}\left(\delta \hat{\mathcal{Z}}_{1, \alpha}+\delta \hat{\mathcal{Z}}_{1, A}\right)+\delta \hat{\mathcal{Z}}_{1, f} \\
\delta \hat{Z}_{2, \mathrm{ffg}}^{(\mathrm{v})}= & \frac{1}{2}\left(\delta \hat{\mathcal{Z}}_{2, \alpha}+\delta \hat{\mathcal{Z}}_{2, A}\right)+\delta \hat{\mathcal{Z}}_{2, f}-\frac{1}{8}\left(\delta \hat{\mathcal{Z}}_{1, \alpha}^{2}+\delta \hat{\mathcal{Z}}_{1, A}^{2}\right)+\frac{1}{2} \delta \hat{\mathcal{Z}}_{1, f}\left(\delta \hat{\mathcal{Z}}_{1, \alpha}+\delta \hat{\mathcal{Z}}_{1, A}\right) \\
& +\frac{1}{4} \delta \hat{\mathcal{Z}}_{1, A} \delta \hat{\mathcal{Z}}_{1, \alpha}
\end{aligned}
$$

and rational counterterms

$$
\begin{aligned}
\delta \hat{\mathcal{R}}_{1, \mathrm{ffg}}^{(\mathrm{v})}= & -2 C_{\mathrm{F}} \\
\delta \hat{\mathcal{R}}_{2, \mathrm{ffg}}^{(\mathrm{v})}= & {\left[-\frac{5}{144} C_{\mathrm{A}}^{2}-\frac{26}{9} C_{\mathrm{A}} C_{\mathrm{F}}+\frac{4}{3} C_{\mathrm{F}}^{2}+\frac{7}{9} T_{\mathrm{F}} n_{\mathrm{f}}\left(C_{\mathrm{A}}+C_{\mathrm{F}}\right)\right] \varepsilon^{-1} } \\
& +\frac{829}{864} C_{\mathrm{A}}^{2}-\frac{563}{54} C_{\mathrm{A}} C_{\mathrm{F}}+\frac{109}{18} C_{\mathrm{F}}^{2}-\frac{T_{\mathrm{F}} n_{\mathrm{f}}}{27}\left(7 C_{\mathrm{A}}-\frac{55}{2} C_{\mathrm{F}}\right)-3 C_{\mathrm{F}} \delta \hat{\mathcal{Z}}_{1, \alpha} \\
& +\frac{1}{2}\left(C_{\mathrm{A}}-3 C_{\mathrm{F}}\right) \delta \hat{\mathcal{Z}}_{1, A}+\frac{1}{6}\left(2 C_{\mathrm{A}}+5 C_{\mathrm{F}}\right) \delta \hat{\mathcal{Z}}_{1, \mathrm{gp}}+\frac{1}{6}\left(C_{\mathrm{A}}-8 C_{\mathrm{F}}\right) \delta \hat{\mathcal{Z}}_{1, f}
\end{aligned}
$$

Gauge-boson three-point vertex. For the triple gauge-boson vertex we have

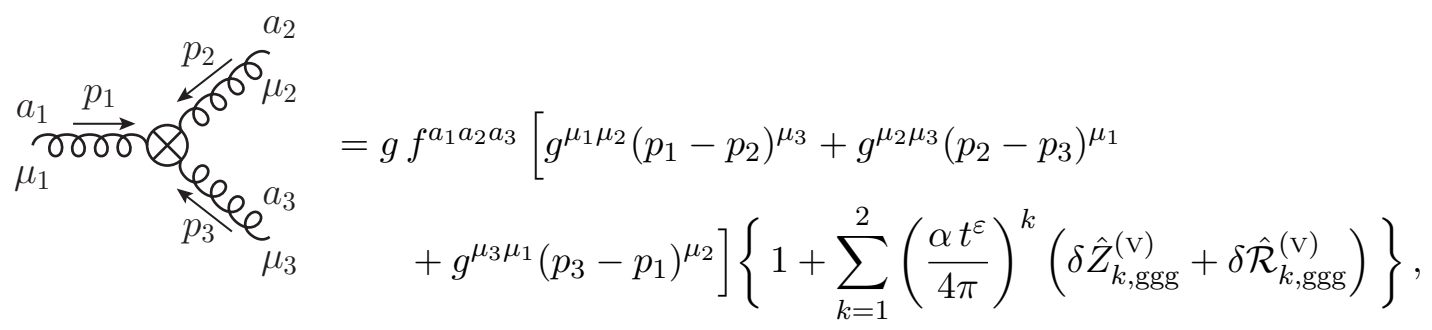

with UV counterterms

$$
\begin{aligned}
& \delta \hat{Z}_{1, \mathrm{ggg}}^{(\mathrm{v})}=\frac{1}{2} \delta \hat{\mathcal{Z}}_{1, \alpha}+\frac{3}{2} \delta \hat{\mathcal{Z}}_{1, A} \\
& \delta \hat{Z}_{2, \mathrm{ggg}}^{(\mathrm{v})}=\frac{1}{2} \delta \hat{\mathcal{Z}}_{2, \alpha}+\frac{3}{2} \delta \hat{\mathcal{Z}}_{2, A}-\frac{1}{8} \delta \hat{\mathcal{Z}}_{1, \alpha}^{2}+\frac{3}{8} \delta \hat{\mathcal{Z}}_{1, A}^{2}+\frac{3}{4} \delta \hat{\mathcal{Z}}_{1, A} \delta \hat{\mathcal{Z}}_{1, \alpha}
\end{aligned}
$$


and rational counterterms

$$
\begin{aligned}
\delta \hat{\mathcal{R}}_{1, \mathrm{ggg}}^{(\mathrm{v})}= & -\frac{11}{12} C_{\mathrm{A}}-\frac{4}{3} T_{\mathrm{F}} n_{\mathrm{f}}, \\
\delta \hat{\mathcal{R}}_{2, \mathrm{ggg}}^{(\mathrm{v})}= & -\left[\frac{11}{48} C_{\mathrm{A}}^{2}+T_{\mathrm{F}} n_{\mathrm{f}}\left(\frac{23}{6} C_{\mathrm{A}}-\frac{8}{3} C_{\mathrm{F}}\right)\right] \varepsilon^{-1}+T_{\mathrm{F}} n_{\mathrm{f}}\left(\frac{25}{9} C_{\mathrm{A}}-\frac{119}{36} C_{\mathrm{F}}\right) \\
& +\frac{145}{288} C_{\mathrm{A}}^{2}-\left(\frac{11}{8} C_{\mathrm{A}}+2 T_{\mathrm{F}} n_{\mathrm{f}}\right) \delta \hat{\mathcal{Z}}_{1, \alpha}-\left(\frac{13}{4} C_{\mathrm{A}}+2 T_{\mathrm{F}} n_{\mathrm{f}}\right) \delta \hat{\mathcal{Z}}_{1, A} \\
& +\frac{5}{4} C_{\mathrm{A}} \delta \hat{\mathcal{Z}}_{1, \mathrm{gp}}-\frac{C_{\mathrm{A}}}{24} \delta \hat{\mathcal{Z}}_{1, u}+\frac{4}{3} T_{\mathrm{F}} \sum_{f \in \mathcal{F}} \delta \hat{\mathcal{Z}}_{1, f}
\end{aligned}
$$

Gauge-boson-ghost three-point vertex. For the gauge-boson-ghost-antighost vertex we have

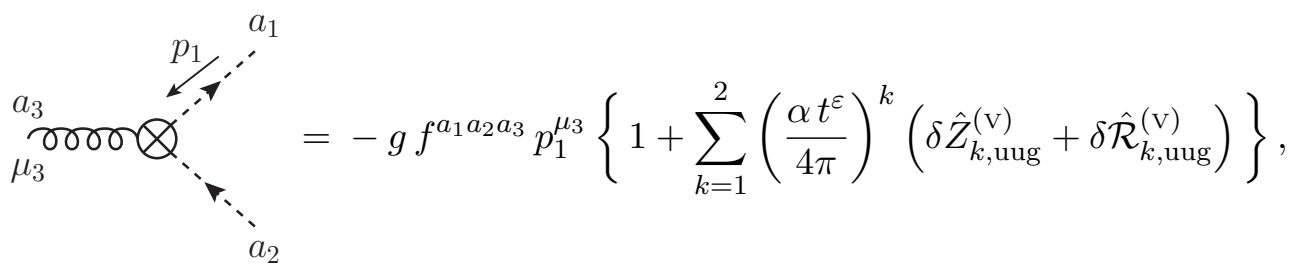

with UV counterterms

$$
\begin{aligned}
\delta \hat{Z}_{1, \text { uug }}^{(\mathrm{v})}= & \frac{1}{2}\left(\delta \hat{\mathcal{Z}}_{1, \alpha}+\delta \hat{\mathcal{Z}}_{1, A}\right)+\delta \hat{\mathcal{Z}}_{1, u}, \\
\delta \hat{Z}_{2, \text { uug }}^{(\mathrm{v})}= & \frac{1}{2}\left(\delta \hat{\mathcal{Z}}_{2, \alpha}+\delta \hat{\mathcal{Z}}_{2, A}\right)+\delta \hat{\mathcal{Z}}_{2, u}-\frac{1}{8}\left(\delta \hat{\mathcal{Z}}_{1, \alpha}^{2}+\delta \hat{\mathcal{Z}}_{1, A}^{2}\right)+\frac{1}{2} \delta \hat{\mathcal{Z}}_{1, u}\left(\delta \hat{\mathcal{Z}}_{1, \alpha}+\delta \hat{\mathcal{Z}}_{1, A}\right) \\
& +\frac{1}{4} \delta \hat{\mathcal{Z}}_{1, A} \delta \hat{\mathcal{Z}}_{1, \alpha},
\end{aligned}
$$

and rational counterterms

$$
\begin{aligned}
\delta \hat{\mathcal{R}}_{1, \text { uug }}^{(\mathrm{v})}= & -\frac{C_{\mathrm{A}}}{4} \\
\delta \hat{\mathcal{R}}_{2, \text { uug }}^{(\mathrm{v})}= & -\left(\frac{7}{36} C_{\mathrm{A}}^{2}-\frac{5}{36} T_{\mathrm{F}} n_{\mathrm{f}} C_{\mathrm{A}}\right) \varepsilon^{-1}-\frac{107}{432} C_{\mathrm{A}}^{2}-\frac{19}{216} T_{\mathrm{F}} n_{\mathrm{f}} C_{\mathrm{A}} \\
& -C_{\mathrm{A}}\left(\frac{3}{8} \delta \hat{\mathcal{Z}}_{1, \alpha}+\frac{1}{4} \delta \hat{\mathcal{Z}}_{1, A}-\frac{5}{12} \delta \hat{\mathcal{Z}}_{1, \mathrm{gp}}+\frac{1}{24} \delta \hat{\mathcal{Z}}_{1, u}\right) .
\end{aligned}
$$

Gauge-boson four-point vertex. For the quartic-gluon vertex we find

$$
\begin{aligned}
& a_{1}^{a_{1}} 2_{2} 6^{6 \mu_{2}}=\mathrm{i} g^{2} \sum_{\pi(234)}^{a_{2}}\left\{f^{a_{1} a_{3} e} f^{a_{2} a_{4} e} \mathcal{V}_{\mathrm{I}}^{\mu_{1} \mu_{2} \mu_{3} \mu_{4}}\left[1+\sum_{k=1}^{2}\left(\frac{\alpha t^{\varepsilon}}{4 \pi}\right)^{k} \delta \hat{Z}_{k, 4 \mathrm{~g}}^{(\mathrm{AI})}\right]\right. \\
& \left.+\sum_{k=1}^{2}\left(\frac{\alpha t^{\varepsilon}}{4 \pi}\right)^{k}\left[\sum_{\beta=\mathrm{I}, \mathrm{II}}\left(T_{\mathrm{F}} \delta^{a_{1} a_{2}} \delta^{a_{3} a_{4}} \delta \hat{\mathcal{R}}_{k, 4 \mathrm{~g}}^{(\mathrm{B} \beta)}+\frac{\operatorname{Tr}\left(T^{a_{1}} T^{a_{3}} T^{a_{2}} T^{a_{4}}\right)}{T_{\mathrm{F}}} \delta \hat{\mathcal{R}}_{k, 4 \mathrm{~g}}^{(\mathrm{C} \beta)}\right) \mathcal{V}_{\beta}^{\mu_{1} \mu_{2} \mu_{3} \mu_{4}}\right]\right\},
\end{aligned}
$$


where

$$
\mathcal{V}_{\mathrm{I}}^{\mu_{1} \mu_{2} \mu_{3} \mu_{4}}=g^{\mu_{1} \mu_{2}} g^{\mu_{3} \mu_{4}}, \quad \mathcal{V}_{\mathrm{II}}^{\mu_{1} \mu_{2} \mu_{3} \mu_{4}}=g^{\mu_{1} \mu_{3}} g^{\mu_{2} \mu_{4}}+g^{\mu_{1} \mu_{4}} g^{\mu_{2} \mu_{3}},
$$

and $\pi(234)$ denotes the six permutations of the particle labels 234 . Note that the Lorentz tensors (5.31) are separately invariant w.r.t. $1 \leftrightarrow 2$ and $3 \leftrightarrow 4$. Thus the sum over $\pi(234)$ generates only three independent Lorentz tensors for each $\mathcal{V}_{\beta}^{\mu_{1} \mu_{2} \mu_{3} \mu_{4}}$. When these Lorentz tensors are combined with the various group-theoretical structures in (5.30), i.e.

$$
\mathcal{C}_{\mathrm{A}}^{a_{1} a_{2} a_{3} a_{4}}=f^{a_{1} a_{3} e} f^{a_{2} a_{4} e}, \quad \mathcal{C}_{\mathrm{B}}^{a_{1} a_{2} a_{3} a_{4}}=T_{\mathrm{F}} \delta^{a_{1} a_{2}} \delta^{a_{3} a_{4}}, \quad \mathcal{C}_{\mathrm{C}}^{a_{1} a_{2} a_{3} a_{4}}=\frac{\operatorname{Tr}\left(T^{a_{1}} T^{a_{3}} T^{a_{2}} T^{a_{4}}\right)}{T_{\mathrm{F}}},
$$

the summation of each combination $\mathcal{C}_{\alpha}^{a_{1} a_{2} a_{3} a_{4}} \mathcal{V}_{\beta}^{\mu_{1} \mu_{2} \mu_{3} \mu_{4}}$ over $\pi(234)$ yields,

$$
\sum_{\pi(234)} \mathcal{C}_{\alpha}^{a_{1} a_{2} a_{3} a_{4}} \mathcal{V}_{\beta}^{\mu_{1} \mu_{2} \mu_{3} \mu_{4}}=\left[\mathcal{C}_{\alpha}^{a_{1} a_{2} a_{3} a_{4}}+\mathcal{C}_{\alpha}^{a_{1} a_{2} a_{4} a_{3}}\right] \mathcal{V}_{\beta}^{\mu_{1} \mu_{2} \mu_{3} \mu_{4}}+(2 \leftrightarrow 3)+(2 \leftrightarrow 4),
$$

where each of the three terms on the r.h.s. is separately invariant w.r.t. $1 \leftrightarrow 2$ and $3 \leftrightarrow 4$. As a consequence (5.33) is totally symmetric in the four particle indices 1234 . Note that the ordering of the generators in the trace of the gauge-group structure $\mathcal{C}_{\mathrm{C}}^{a_{1} a_{2} a_{3} a_{4}}$ in (5.30) and (5.32) is $T^{a_{1}} T^{a_{3}} T^{a_{2}} T^{a_{4}}$. Note also that in the definition of $\mathcal{C}_{\mathrm{B}}$ and $\mathcal{C}_{\mathrm{C}}$ we include explicit $T_{\mathrm{F}}$ factors in such a way that all gauge-group structures in (5.32) scale like $T_{\mathrm{F}}$.

The UV counterterms for the quartic vertex (5.30) read

$$
\begin{aligned}
& \delta \hat{Z}_{1,4 \mathrm{~g}}^{(\mathrm{AI})}=\delta \hat{\mathcal{Z}}_{1, \alpha}+2 \delta \hat{\mathcal{Z}}_{1, A}, \\
& \delta \hat{\mathcal{Z}}_{2,4 \mathrm{~g}}^{(\mathrm{AI})}=\delta \hat{\mathcal{Z}}_{2, \alpha}+2 \delta \hat{\mathcal{Z}}_{2, A}+\delta \hat{\mathcal{Z}}_{1, A}^{2}+2 \delta \hat{\mathcal{Z}}_{1, \alpha} \delta \hat{\mathcal{Z}}_{1, A},
\end{aligned}
$$

and for the rational counterterms we find

$$
\begin{aligned}
\delta \hat{\mathcal{R}}_{1,4 \mathrm{~g}}^{(\mathrm{BI})=} & -\frac{1}{3} \frac{C_{\mathrm{A}}}{N} \\
\delta \hat{\mathcal{R}}_{2,4 \mathrm{~g}}^{(\mathrm{BI})=} & \frac{C_{\mathrm{A}}}{2}\left[\left(\frac{13}{12}+\frac{1}{6} \frac{n_{\mathrm{f}}}{N}\right) T_{\mathrm{F}} \varepsilon^{-1}-\left(\frac{571}{288}-\frac{53}{36} \frac{n_{\mathrm{f}}}{N}\right) T_{\mathrm{F}}-\frac{1}{N}\left(\frac{4}{3} \delta \hat{\mathcal{Z}}_{1, \alpha}+\frac{29}{24} \delta \hat{\mathcal{Z}}_{1, A}\right.\right. \\
& \left.\left.+\frac{1}{4} \delta \hat{\mathcal{Z}}_{1, u}-\frac{1}{8} \delta \hat{\mathcal{Z}}_{1, \mathrm{gp}}\right)\right], \\
\delta \hat{\mathcal{R}}_{1,4 \mathrm{~g}}^{(\mathrm{BII})=} & -\frac{1}{3} \frac{C_{\mathrm{A}}}{N} \\
\delta \hat{\mathcal{R}}_{2,4 \mathrm{~g}}^{(\mathrm{BII})}= & \frac{C_{\mathrm{A}}}{2}\left[\left(-\frac{23}{12}+\frac{7}{6} \frac{n_{\mathrm{f}}}{N}\right) T_{\mathrm{F}} \varepsilon^{-1}+\left(\frac{233}{72}+\frac{23}{36} \frac{n_{\mathrm{f}}}{N}\right) T_{\mathrm{F}}-\frac{1}{N}\left(\frac{4}{3} \delta \hat{\mathcal{Z}}_{1, \alpha}+\frac{29}{24} \delta \hat{\mathcal{Z}}_{1, A}\right.\right. \\
& \left.\left.+\frac{1}{4} \delta \hat{\mathcal{Z}}_{1, u}-\frac{1}{8} \delta \hat{\mathcal{Z}}_{1, \mathrm{gp}}\right)\right],
\end{aligned}
$$




$$
\begin{aligned}
\delta \hat{\mathcal{R}}_{1,4 \mathrm{~g}}^{(\mathrm{CI})}= & -\frac{8}{3} C_{\mathrm{A}}-\frac{10}{3} T_{\mathrm{F}} n_{\mathrm{f}} \\
\delta \hat{\mathcal{R}}_{2,4 \mathrm{~g}}^{(\mathrm{CI})}= & -\left[\frac{271}{144} C_{\mathrm{A}}^{2}+2 \frac{C_{\mathrm{A}} T_{\mathrm{F}}}{N}+T_{\mathrm{F}} n_{\mathrm{f}}\left(\frac{247}{36} C_{\mathrm{A}}-\frac{20}{3} C_{\mathrm{F}}\right)\right] \varepsilon^{-1}-\frac{421}{3456} C_{\mathrm{A}}^{2} \\
& +\frac{167}{48} \frac{C_{\mathrm{A}} T_{\mathrm{F}}}{N}+T_{\mathrm{F}} n_{\mathrm{f}}\left(\frac{199}{27} C_{\mathrm{A}}-\frac{43}{6} C_{\mathrm{F}}\right)-\left(\frac{16}{3} C_{\mathrm{A}}+\frac{20}{3} T_{\mathrm{F}} n_{\mathrm{f}}\right) \delta \hat{\mathcal{Z}}_{1, \alpha} \\
& -\left(\frac{379}{48} C_{\mathrm{A}}+\frac{20}{3} T_{\mathrm{F}} n_{\mathrm{f}}\right) \delta \hat{\mathcal{Z}}_{1, A}-\frac{C_{\mathrm{A}}}{24} \delta \hat{\mathcal{Z}}_{1, u}+\frac{157}{48} C_{\mathrm{A}} \delta \hat{\mathcal{Z}}_{1, \mathrm{gp}}+\frac{8}{3} T_{\mathrm{F}} \sum_{f \in \mathcal{F}} \delta \hat{\mathcal{Z}}_{1, f}, \\
\delta \hat{\mathcal{R}}_{1,4 \mathrm{~g}}^{(\mathrm{CII})}= & \frac{7}{6} C_{\mathrm{A}}+2 T_{\mathrm{F}} n_{\mathrm{f}} \\
\delta \hat{\mathcal{R}}_{2,4 \mathrm{~g}}^{(\mathrm{CII})}= & {\left[\frac{119}{144} C_{\mathrm{A}}^{2}+\frac{C_{\mathrm{A}} T_{\mathrm{F}}}{N}+T_{\mathrm{F}} n_{\mathrm{f}}\left(\frac{143}{36} C_{\mathrm{A}}-\frac{10}{3} C_{\mathrm{F}}\right)\right] \varepsilon^{-1}+\frac{857}{3456} C_{\mathrm{A}}^{2} } \\
& -\frac{167}{96} \frac{C_{\mathrm{A}} T_{\mathrm{F}}}{N}-T_{\mathrm{F}} n_{\mathrm{f}}\left(\frac{1649}{432} C_{\mathrm{A}}-\frac{17}{6} C_{\mathrm{F}}\right)+\left(\frac{7}{3} C_{\mathrm{A}}+4 T_{\mathrm{F}} n_{\mathrm{f}}\right) \delta \hat{\mathcal{Z}}_{1, \alpha} \\
& +\left(\frac{175}{48} C_{\mathrm{A}}+4 T_{\mathrm{F}} n_{\mathrm{f}}\right) \delta \hat{\mathcal{Z}}_{1, A}-\frac{C_{\mathrm{A}}}{24} \delta \hat{\mathcal{Z}}_{1, u}-\frac{77}{48} C_{\mathrm{A}} \delta \hat{\mathcal{Z}}_{1, \mathrm{gp}}-\frac{4}{3} T_{\mathrm{F}} \sum_{f \in \mathcal{F}} \delta \hat{\mathcal{Z}}_{1, f} .
\end{aligned}
$$

Note that for $\mathrm{U}(1)$ gauge theories the coefficients (5.35)-(5.36) vanish.

When using the $\overline{\mathrm{MS}}$ renormalisation constants listed in appendix $\mathrm{B}$ and applying the $\mathrm{U}(1)$ substitutions of table 5.2, all results presented in this section agree with previous results for QED rational counterterms in the $\lambda=1$ gauge [1].

\section{Conclusions}

The most widely used tools for the automated calculation of one-loop amplitudes are based on numerical algorithms that build the numerators of loop integrands in $D_{\mathrm{n}}=4$ dimensions, while the remnant parts are reconstructed by means of rational counterterms. This approach has proven to be very flexible and efficient for the automation of NLO calculations, and its extension to two loops can become an important ingredient in the development of automated tools at NNLO. As a first step in this direction, in [1] it was shown that renormalised two-loop amplitudes in $D=4-2 \varepsilon$ dimensions can be related to corresponding amplitudes in $D_{\mathrm{n}}=4$ dimensions, i.e. with four-dimensional loop numerators, making use of process-independent rational counterterms. More precisely, all two-loop contributions stemming from the interplay of UV poles with the $(D-4)$-dimensional parts of loop numerators can be reconstructed through insertions of the well known one-loop rational counterterms $\delta \mathcal{R}_{1}$ into one-loop amplitudes and insertions of two-loop rational counterterms $\delta \mathcal{R}_{2}$ into tree amplitudes. In addition, for the subtraction of one-loop subdivergences in $D_{\mathrm{n}}=4$ dimensions the usual UV counterterms $\delta Z_{1}$ need to be supplemented by extra counterterms $\delta \tilde{Z}_{1}$ proportional to $\tilde{q}^{2} / \varepsilon$.

In this paper we have presented a general analysis of the dependence of two-loop rational terms on the choice of renormalisation scheme. Specifically we have demonstrated that the form of the master formula for renormalised two-loop amplitudes - initially 
established within the minimal subtraction scheme - is independent of the renormalisation scheme. Moreover we have derived general formulas (4.62)-(4.63) that describe the scheme dependence of $\delta \mathcal{R}_{2}$ counterterms as the combination of two contributions: the naive renormalisation of $\delta \mathcal{R}_{1}$ counterterms and an extra nontrivial contribution, which is due to the fact that the multiplicative renormalisation of subdivergences does not commute with the projection of loop numerators to $D_{\mathrm{n}}=4$ dimensions. In renormalisable theories, such nontrivial contributions originate only from one-loop selfenergy subdiagrams and can be controlled through auxiliary one-loop counterterms as specified in (4.100)-(4.104).

As a consequence, the scheme-dependent part of $\delta \mathcal{R}_{2}$ counterterms can be expressed as a linear combination of one-loop renormalisation constants with process- and schemeindependent coefficients. This makes it possible to derive the $\delta \mathcal{R}_{2}$ counterterms for a given renormalisable theory in terms of generic one-loop renormalisation constants, which can be adapted a posteriori to any desired scheme.

Using the above approach we have generalised the known $\delta \mathcal{R}_{2}$ counterterms for QED from the minimal subtraction scheme to any renormalisation scheme. Moreover, we have presented the first calculation of the full set of $\delta \mathcal{R}_{2}$ counterterms for Yang-Mills theories. All calculations have been carried out in the Feynman gauge, and the results are presented in compact formulas that are applicable to $\mathrm{SU}(\mathrm{N})$ or $\mathrm{U}(1)$ theories with $n_{\mathrm{f}}$ massless or massive fermions.

Technically, for the calculation of $\delta \mathcal{R}_{2}$ counterterms we have used various expansions that capture the UV divergences of all relevant one- and two-loop diagrams in the form of massive tadpole integrals. Such tadpole expansions are described in detail in appendix A, including the expansions employed in [1] as well as a new variant that reduces the number of expansion terms and allows also for a fully flexible parametrisation of loop momenta.

In the future we plan to investigate two-loop rational terms within spontaneously broken gauge theories and to study the interplay of rational terms with infrared divergences.

\section{Acknowledgments}

This research was supported by the Swiss National Science Foundation (SNSF) under contract BSCGI0-157722. The work of M.Z. was supported through the SNSF Ambizione grant PZ00P2-179877.

\section{A Tadpole expansions}

In this appendix we review the techniques that have been used in [1] to express rational terms in the form of massive tadpole integrals and we present various optimisations.

\section{A.1 Iterative tadpole decomposition}

The UV poles of multi-loop integrals and the associated rational parts can be isolated via recursive decomposition of the loop propagators by means of the partial-fractioning 
formula [49-51]

$$
\frac{1}{D_{a}^{(i)}\left(\bar{q}_{i}\right)}=\frac{1}{\bar{q}_{i}^{2}-M^{2}}+\frac{\Delta_{i a}\left(\bar{q}_{i}\right)}{\bar{q}_{i}^{2}-M^{2}} \frac{1}{D_{a}^{(i)}\left(\bar{q}_{i}\right)}
$$

where the denominator $D_{a}^{(i)}\left(\bar{q}_{i}\right)$ is defined in (3.3), and

$$
\Delta_{i a}\left(\bar{q}_{i}\right)=\left(\bar{q}_{i}^{2}-M^{2}\right)-D_{a}^{(i)}\left(\bar{q}_{i}\right)=-p_{i a}^{2}-2 \bar{q}_{i} \cdot p_{i a}+m_{i a}^{2}-M^{2},
$$

while $M$ is an auxiliary mass scale. The above formula splits a generic scalar propagator into a tadpole propagator $1 /\left(\bar{q}_{i}^{2}-M^{2}\right)$ and a remnant part that consists of the original propagator times a factor $\Delta_{i a}\left(\bar{q}_{i}\right) /\left(\bar{q}_{i}^{2}-M^{2}\right)$, which is suppressed by $\mathcal{O}\left(1 / \bar{q}_{i}\right)$ in the UV limit $\bar{q}_{i} \rightarrow \infty$.

Iterating the above identity $X_{i}+1$ times makes it possible to capture all UV contributions of the propagator $1 / D_{a}^{(i)}\left(\bar{q}_{i}\right)$ up to relative order $1 / \bar{q}_{i}^{X_{i}}$ in the form of tadpole integrands. This procedure can be easily extended to chains of $\bar{q}_{i}$-dependent scalar propagators (3.2). To this end the identity (A.1) should be iterated on all $N_{i}$ propagators in the chain, and terms with denominators of the form $\left(\bar{q}_{i}^{2}-M^{2}\right)^{p} D_{a_{1}}^{(i)}\left(\bar{q}_{i}\right) \cdots D_{a_{r}}^{(i)}\left(\bar{q}_{i}\right)$ with $p+r>N_{i}+X_{i}$ should be discarded. This algorithm can be encoded in a tadpole expansion operator $\mathbf{S}_{X_{i}}^{(i)}$, which yields combinations of tadpole integrands

$$
\mathbf{S}_{X_{i}}^{(i)} \frac{1}{D_{0}^{(i)}\left(\bar{q}_{i}\right) \cdots D_{N_{i}-1}^{(i)}\left(\bar{q}_{i}\right)}=\sum_{\sigma=0}^{X_{i}} \frac{\Delta_{i}^{(\sigma)}\left(\bar{q}_{i}\right)}{\left(\bar{q}_{i}^{2}-M^{2}\right)^{N_{i}+\sigma}},
$$

where the numerators on the r.h.s. read

$$
\Delta_{i}^{(\sigma)}\left(\bar{q}_{i}\right)=\left.\sum_{\sigma_{0}=0}^{\sigma} \ldots \sum_{\sigma_{N_{i}-1}=0}^{\sigma} \prod_{a=0}^{N_{i}-1}\left[\Delta_{i a}\left(\bar{q}_{i}\right)\right]^{\sigma_{a}}\right|_{\sigma_{0}+\cdots+\sigma_{N_{i}-1}=\sigma},
$$

and correspond to polynomials of homogeneous degree $\sigma$ in $\bar{q}_{i} \cdot p_{i a}$ and in the squared mass scales $\left\{p_{i a}^{2}, m_{i a}^{2}\right\}$ and $M^{2}$.

By construction, the tadpole integrands on the r.h.s. of (A.3) capture the leading and subleading UV contributions of the original propagator chain up to relative order $1 / \bar{q}_{i}^{X_{i}}$. Thus, formally

$$
1-\mathbf{S}_{X_{i}}^{(i)}=\mathcal{O}\left(1 / \bar{q}_{i}^{X_{i}+1}\right)
$$

Note that suppressed contributions of order $1 / \bar{q}_{i}^{X_{i}+1}$ and beyond are present also in (A.3). This is due to the fact that terms of $\mathcal{O}\left(\bar{q}_{i}^{1}\right)$ and $\mathcal{O}\left(\bar{q}_{i}^{0}\right)$ in (A.2) are treated on the same footing. Possible optimisations that minimise the number of irrelevant higher-order terms in $1 / \bar{q}_{i}$ are discussed in the subsequent sections.

For loop chains,

$$
\overline{\mathcal{F}}_{\bar{\alpha}_{i}}^{(i)}\left(\bar{q}_{i}\right)=\frac{\overline{\mathcal{N}}_{\bar{\alpha}_{i}}^{(i)}\left(\bar{q}_{i}\right)}{\mathcal{D}^{(i)}\left(\bar{q}_{i}\right)}
$$


where $\overline{\mathcal{N}}_{\bar{\alpha}_{i}}^{(i)}$ and $\mathcal{D}^{(i)}\left(\bar{q}_{i}\right)$ are defined in (3.2) and (3.4), the $\mathbf{S}_{X_{i}}^{(i)}$ expansion can be defined as

$$
\mathbf{S}_{X_{i}}^{(i)} \overline{\mathcal{F}}_{\bar{\alpha}_{i}}^{(i)}\left(\bar{q}_{i}\right)=\overline{\mathcal{N}}_{\bar{\alpha}_{i}}^{(i)}\left(\bar{q}_{i}\right) \mathbf{S}_{X_{i}}^{(i)}\left(\frac{1}{\mathcal{D}^{(i)}\left(\bar{q}_{i}\right)}\right)
$$

where the loop numerator is kept unexpanded. For one-loop diagrams,

$$
\overline{\mathcal{A}}_{1, \Gamma}=\int \mathrm{d} \bar{q}_{1} \overline{\mathcal{F}}^{(1)}\left(\bar{q}_{1}\right)
$$

we define

$$
\overline{\mathcal{A}}_{1, \Gamma_{\text {tad }}}=\mathbf{S}_{X_{1}}^{(1)} \overline{\mathcal{A}}_{1, \Gamma}=\int \mathrm{d} \bar{q}_{1} \mathbf{S}_{X_{1}}^{(1)} \overline{\mathcal{F}}^{(1)}\left(\bar{q}_{1}\right),
$$

where the order $X_{1}$ of the expansion should be set equal to the degree of divergence of $\Gamma$. Due to (A.5), this choice guarantees that $\overline{\mathcal{A}}_{1, \Gamma_{\text {rem }}}=\overline{\mathcal{A}}_{1, \Gamma_{\text {tad }}}-\overline{\mathcal{A}}_{1, \Gamma}$ has negative degree of divergence, which implies that all UV divergences of $\Gamma$ are embodied in the tadpole expansion (A.9).

At two loops, UV divergences can be isolated in tadpole integrals by means of three separate tadpole expansions $\mathbf{S}_{X_{i}}^{(i)}$ with $i=1,2,3$, each of which acts exclusively on the $\bar{q}_{i^{-}}$ dependent chain $\mathcal{C}_{i}$. More explicitly, for the generic two-loop diagram (3.1) one can define

$$
\overline{\mathcal{A}}_{2, \Gamma_{\text {tad }}}=\prod_{i=1}^{3} \mathbf{S}_{X_{i}}^{(i)} \overline{\mathcal{A}}_{2, \Gamma}=\int \mathrm{d} \bar{q}_{1} \int \mathrm{d} \bar{q}_{2}\left[\bar{\Gamma}^{\bar{\alpha}_{1} \bar{\alpha}_{2} \bar{\alpha}_{3}}\left(\bar{q}_{1}, \bar{q}_{2}, \bar{q}_{3}\right) \prod_{i=1}^{3}\left(\mathbf{S}_{X_{i}}^{(i)} \overline{\mathcal{F}}_{\bar{\alpha}_{i}}^{(i)}\left(\bar{q}_{i}\right)\right)\right]_{\bar{q}_{3}=-\bar{q}_{1}-\bar{q}_{2}}{ }_{\text {(A.10) }}
$$

Here the order of the various $\mathbf{S}_{X_{i}}^{(i)}$ expansions should be chosen as

$$
X_{i}=X_{i}(\Gamma)=\max \left\{X(\Gamma), X_{i j}(\Gamma), X_{i k}(\Gamma)\right\}
$$

where $X(\Gamma)$ is the global degree of divergence, $i \mid j k$ is a partition of 123 , and $X_{i m}(\Gamma)$ with $m=j, k$ are the degrees of divergence of the subdiagrams that contain the chain $\mathcal{C}_{i}$, i.e. the subdiagrams that are subject to the $\mathbf{S}_{X_{i}}^{(i)}$ expansion. With this choice, for each individual $\mathbf{S}_{X_{i}}^{(i)}$ expansion the discarded $\left(1-\mathbf{S}_{X_{i}}^{(i)}\right)$ contribution of order $1 / \bar{q}^{X_{i}+1}$ has a global degree of divergence $X(\Gamma)-X_{i}(\Gamma)<0$, and a degree of subdivergence $X_{i m}(\Gamma)-X_{i}(\Gamma)<0$ for the subdiagrams that are subject to the $\mathbf{S}_{X_{i}}^{(i)}$ expansion. This means that each $\mathbf{S}_{X_{i}}^{(i)}$ expansion retains the full local divergence as well as the full divergences of the two subdiagrams that contain the chain $\mathcal{C}_{i}$. For what concerns the subdiagram $\gamma_{i}$, which does not contain the chain $\mathcal{C}_{i}$, its subdivergence factorises w.r.t. the $\mathbf{S}_{X_{i}}^{(i)}$ expansion. This implies that also the $\left(1-\mathbf{S}_{X_{i}}^{(i)}\right)$ finite remnant of the expansion of the chain $\mathcal{C}_{i}$ factorises w.r.t. the $\gamma_{i}$ subdivergence. As a result, the remnant of the complete expansion (A.10) still contains the divergent parts

$$
\overline{\mathcal{A}}_{2, \Gamma_{\text {rem }, \text { div }}}=\left.\sum_{i=1}^{3}\left(1-\mathbf{S}_{X_{i}}^{(i)}\right) \mathbf{S}_{X_{j}}^{(j)} \mathbf{S}_{X_{k}}^{(k)} \overline{\mathcal{A}}_{2, \Gamma}\right|_{\{j, k\}=\{1,2,3\} \backslash\{i\}},
$$

which involve the full subdivergence of the various $\gamma_{i}$ subdiagrams combined with the remnants of the expansions of their complementary chains $\mathcal{C}_{i}$. These missing UV divergent parts are not globally divergent. Thus, according to (3.15), they do not contribute to $\delta \mathcal{R}_{2, \Gamma}$. 
As for the tadpole expansion (A.10), as discussed above it matches the full local divergence of $\Gamma$ as well as the divergences of its individual subdiagrams, thereby fulfilling the requirements (3.18) and (3.21). Thus two-loop rational terms can be computed using the formulas (3.19) and (3.22). More explicitly,

$$
\begin{aligned}
\delta \mathcal{R}_{2, \Gamma}= & \int \mathrm{d} \bar{q}_{1} \int \mathrm{d} \bar{q}_{2}\left[\bar{\Gamma}^{\bar{\alpha}_{1} \bar{\alpha}_{2} \bar{\alpha}_{3}}\left(\bar{q}_{1}, \bar{q}_{2}, \bar{q}_{3}\right) \prod_{i=1}^{3}\left(\mathbf{S}_{X_{i}}^{(i)} \overline{\mathcal{F}}_{\bar{\alpha}_{i}}^{(i)}\left(\bar{q}_{i}\right)\right)-\Gamma^{\alpha_{1} \alpha_{2} \alpha_{3}}\left(q_{1}, q_{2}, q_{3}\right)\right. \\
& \left.\times \prod_{i=1}^{3}\left(\mathbf{S}_{X_{i}}^{(i)} \mathcal{F}_{\alpha_{i}}^{(i)}\left(q_{i}\right)\right)\right]_{q_{3}=-q_{1}-q_{2}}+\sum_{i=1}^{3} \int \mathrm{d} \bar{q}_{i}\left[\delta Z_{1, \gamma_{i}}^{\bar{\alpha}_{i}}\left(\bar{q}_{i}\right) \mathbf{S}_{X_{i}}^{(i)} \overline{\mathcal{F}}_{\bar{\alpha}_{i}}^{(i)}\left(\bar{q}_{i}\right)\right. \\
& \left.-\left(\delta Z_{1, \gamma_{i}}^{\alpha_{i}}\left(q_{i}\right)+\delta \tilde{Z}_{1, \gamma_{i}}^{\alpha_{i}}\left(\tilde{q}_{i}\right)+\delta \mathcal{R}_{1, \gamma_{i}}^{\alpha_{i}}\left(q_{i}\right)\right) \mathbf{S}_{X_{i}}^{(i)} \mathcal{F}_{\alpha_{i}}^{(i)}\left(q_{i}\right)\right]
\end{aligned}
$$

where

$$
\mathcal{F}_{\alpha_{i}}^{(i)}\left(q_{i}\right)=\frac{\mathcal{N}_{\alpha_{i}}^{(i)}\left(q_{i}\right)}{\mathcal{D}^{(i)}\left(\bar{q}_{i}\right)}
$$

is the projection of the chain (A.6) to $D_{\mathrm{n}}=4$ dimensions. The orders $X_{i}$ for the expansions of the various chains $\mathcal{C}_{i}$ in (A.13) have to be chosen according to (A.11). In the presence of UV divergent subdiagrams, their determination can be facilitated by observing that ${ }^{9}$

$$
X_{i}(\Gamma)=\max \left\{X(\Gamma), X_{i j}(\Gamma), X_{i k}(\Gamma)\right\}=X(\Gamma) \quad \text { if } \quad X\left(\gamma_{i}\right)=X_{j k}(\Gamma) \geq 0 .
$$

This relation is especially useful for the one-loop integrals with $\delta Z_{1, \gamma_{i}}, \delta \tilde{Z}_{1, \gamma_{i}}$ and $\delta \mathcal{R}_{1, \gamma_{i}}$ insertions, since such counterterms are non-vanishing only for divergent subdiagrams $\gamma_{i}$, i.e. when $X\left(\gamma_{i}\right) \geq 0$. Thus, according to (A.16), the order of the expansion of the complementary chain $\mathcal{C}_{i}$ is simply given by $X_{i}=X(\Gamma)$ and does not depend on the details of the two chains $\mathcal{C}_{j}, \mathcal{C}_{k}$ inside $\gamma_{i}$.

In (A.13) rational terms arise from the interplay of the $(D-4)$-dimensional parts of the numerators with UV singularities, and by construction only local divergences contribute, while subdivergences cancel out. This property has various important implications. First of all it makes it possible to discard the divergent parts (A.12), and thus to reduce $\delta \mathcal{R}_{2, \Gamma}$ terms to tadpole integrals. Moreover, it guarantees that all terms depending on the auxiliary tadpole mass $M$ cancel in (A.13). This cancellation mechanism can be understood by observing that, before applying the $\mathbf{S}_{X_{i}}^{(i)}$ expansion, the original integrals are independent of $M$. This implies that the truncated expansions $\mathbf{S}_{X_{i}}^{(i)}$ and their remnants $\left(1-\mathbf{S}_{X_{i}}^{(i)}\right)$ must have identical $M$-dependent parts with opposite signs. Moreover, we know that the remnants do not contribute to $\delta \mathcal{R}_{2, \Gamma}$ since their divergent parts (A.12) are free from local divergences, and non-divergent parts cannot generate rational terms. For this reason, also the $M$ dependent parts of the truncated tadpole expansion (A.10) must cancel in (A.13). In

\footnotetext{
${ }^{9}$ The relation (A.16) is a direct consequence of the inequalities

$$
X(\Gamma) \geq X_{j k}(\Gamma)+X_{i m}(\Gamma) \quad \text { for } \quad m=j, k,
$$
}

where $i j k$ is a permutation of 123 . See section 5.2 of [1]. 
practice, the cancellation of the $M$-dependence in (A.13) is guaranteed by the fact that all UV poles and rational parts stemming from the subdivergences of two-loop amplitudes are compensated by the corresponding counterterm insertions in the last two lines of (A.13).

\section{A.2 Power counting in $1 / \bar{q}_{i}$ and parametrisation dependence}

The tadpole expansion defined in the previous section makes it possible to capture all terms up to relative order $1 / \bar{q}_{i}^{X_{i}}$ in a very simple way, namely by iterating (A.1) on the denominators of a loop chain. However, as mentioned above, this naive expansion does not retain only the required terms of $\mathcal{O}\left(1 / \bar{q}_{i}^{X_{i}}\right)$ and lower, but also many unnecessary terms of $\mathcal{O}\left(1 / \bar{q}_{i}^{\left(X_{i}+1\right)}\right)$ and higher.

The number of terms, and related tadpole integrals to be computed, can be reduced in a drastic way by applying a strict power counting in $1 / \bar{q}_{i}$. In practice the expansion (A.7) can be redefined as

$$
\mathbf{S}_{X_{i}}^{(i)} \overline{\mathcal{F}}_{\bar{\alpha}_{i}}^{(i)}\left(\bar{q}_{i}\right)=\mathbf{P}_{X_{i}}^{(i)}\left[\overline{\mathcal{N}}\left(\bar{q}_{i}\right) \mathbf{S}_{X_{i}}^{(i)}\left(\frac{1}{\mathcal{D}^{(i)}\left(\bar{q}_{i}\right)}\right)\right]
$$

where the operator $\mathbf{P}_{X_{i}}^{(i)}$ truncates all terms beyond relative order $1 / \bar{q}_{i}^{X_{i}}$. With this improvement a large number of irrelevant terms are discarded, while the entire analysis of section A.1, including the formula (A.13), remains valid.

When applying the tadpole expansions (A.17) or (A.7) on the r.h.s. of the $\delta \mathcal{R}_{2}$ formula (A.13), care must be taken that the cancellation of all UV poles and rational terms stemming from subdivergences is not disturbed. This requires a one-to-one correspondence between the expansions that are applied to the two-loop integrals and to the related oneloop integrals with counterterm insertions in (A.13). Let us consider, for example, the counterterm contribution

$$
\sum_{\gamma_{i}} \delta Z_{1, \gamma_{i}} \cdot \overline{\mathcal{A}}_{1, \Gamma_{\text {tad }} / \gamma_{i, \text { tad }}}=\sum_{\gamma_{i}} \int \mathrm{d} \bar{q}_{i} \delta Z_{1, \gamma_{i}}^{\bar{\alpha}_{i}}\left(\bar{q}_{i}\right) \mathbf{S}_{X_{i}}^{(i)} \overline{\mathcal{F}}_{\bar{\alpha}_{i}}^{(i)}\left(\bar{q}_{i}\right),
$$

which embodies the UV singularities of the various subdiagrams $\gamma_{i}$ in $D_{\mathrm{n}}=D$ dimensions. Here the expansion $\mathbf{S}_{X_{i}}^{(i)}$ of the complementary one-loop chain $\mathcal{C}_{i}$ must be identical to the $\mathbf{S}_{X_{i}}^{(i)}$ expansion that is applied to the corresponding two-loop amplitude with $D_{\mathrm{n}}=D$ in (A.13). To this end, the propagators of the two-loop diagrams and the corresponding propagators in the one-loop insertions need to be parametrised in the same way. This is mandatory since, in general, after tadpole expansion the two-loop diagrams and the related one-loop insertions depend on the parametrisation of the loop momenta, and only their combination is parametrisation-independent. This is due to the fact that a loopmomentum shift $\bar{q}_{i} \rightarrow \bar{q}_{i}+\Delta p_{i}$, where $\Delta p_{i}$ is a linear combination of the external momenta, turns each term of fixed order $\left(1 / \bar{q}_{i}\right)^{K}$ into combinations of terms of order $\left(1 / \bar{q}_{i}\right)^{K^{\prime}}$ with $K^{\prime} \geq K$. In the case of the counterterm $\delta Z_{1, \gamma_{i}}^{\bar{\alpha}_{i}}\left(\bar{q}_{i}\right)$ in (A.18) all extra higher-order terms resulting from the shift are retained, while in the case of the chain $\mathcal{F}_{\bar{\alpha}_{i}}^{(i)}\left(\bar{q}_{i}\right)$ they are in part truncated by the $\mathbf{S}_{X_{i}}^{(i)}$ expansion. In general, this results into a dependence on the shift $\Delta p_{i}$. Therefore, changing the parametrisation of two-loop diagrams and corresponding one-loop 
diagrams with one-loop counterterms independently from one another can jeopardise the cancellation of subdivergences in (A.13) and give rise to fake $\delta \mathcal{R}_{2, \Gamma}$ contributions.

In principle, (A.18) may be rendered parametrisation invariant by extending the $1 / \bar{q}_{i}$ expansion to the full integrand, including also the counterterm (see appendix A.5). However, this is not consistent with the method of section A.1, which requires the expansion of each chain $\mathcal{C}_{i}$ to be independent of the complementary subdiagram $\gamma_{i}$. Thus the counterterm should be excluded from the $1 / \bar{q}_{i}$ expansion, and when $\delta Z_{1, \gamma_{i}}\left(q_{i}\right)$ depends on $q_{i}$, i.e. when the subdiagram $\gamma_{i}$ involves a non-logarithmic divergence, then (A.18) is not invariant w.r.t. shifts of $\bar{q}_{i}$.

\section{A.3 Taylor expansion in the external momenta and masses}

In the following we introduce an improved tadpole-expansion approach that renders the calculations more efficient and makes it possible to parametrise two-loop integrals and one-loop counterterm insertions independently from each other. This approach is based on Taylor expansions in the external momenta and internal masses, which correspond to expansions in the dimensionless parameters $\left\{p_{i a} / \bar{q}_{i}\right\},\left\{m_{i a} / \bar{q}_{i}\right\}$ and thus to $1 / \bar{q}_{i}$ expansions at level of loop integrands.

To carry out Taylor expansions in the parameters $\left\{p_{i a}, m_{i a}\right\}$ associated with a certain chain $\mathcal{C}_{i}$ we introduce the rescaled parameters

$$
\hat{p}_{i a}=\lambda_{i} p_{i a}, \quad \hat{m}_{i a}=\lambda_{i} m_{i a}, \quad \text { for } \quad a=0, \ldots, N_{i}-1,
$$

and the associated expansion operators

$$
\mathbf{T}_{K}^{(i)}=\left.\frac{1}{K !}\left(\frac{\mathrm{d}}{\mathrm{d} \lambda_{i}}\right)^{K}\right|_{\lambda_{i}=0} \quad \text { and } \quad \mathbf{T}_{\left[0, X_{i}\right]}^{(i)}=\sum_{K=0}^{X_{i}} \mathbf{T}_{K}^{(i)}
$$

For a function $f\left(\left\{p_{i a}, m_{i a}\right\}\right)$ the terms of fixed order $K$ in $\left\{p_{i a}, m_{i a}\right\}$ are obtained by applying $\mathbf{T}_{K}^{(i)}$ to $f\left(\left\{\hat{p}_{i a}, \hat{m}_{i a}\right\}\right)$, while $\mathbf{T}_{\left[0, X_{i}\right]}^{(i)}$ corresponds to the truncated Taylor expansion up to order $X_{i}$. In the case of loop integrands, the dependence on the external momenta $p_{i a}$ arises only from the internal momenta

$$
\hat{\ell}_{i a}=\bar{q}_{i}+\hat{p}_{i a} .
$$

Thus the $\mathbf{T}_{K}^{(i)}$ operator corresponds to

$$
\left.\frac{1}{K !}\left(\frac{\mathrm{d}}{\mathrm{d} \lambda_{i}}\right)^{K}\right|_{\lambda_{i}=0}=\left.\frac{1}{K !}\left(\sum_{a} p_{i a}^{\mu} \frac{\partial}{\partial \hat{\ell}_{i a}^{\mu}}+\sum_{a} m_{i a}^{\mu} \frac{\partial}{\partial \hat{m}_{i a}^{\mu}}\right)^{K}\right|_{\hat{p}_{i a}=\hat{m}_{i a}=0} .
$$

Contrary to the methods of sections A.1-A.2, the above Taylor expansion generates only scaleless tadpole integrals, since all momenta and masses are set to zero in the denominators. This can be avoided by supplementing each propagator denominator by auxiliary squared mass terms

$$
\hat{M}_{i}^{2}=\left(1-\omega_{i}^{2}\right) M^{2}
$$


Physical amplitudes correspond to $\omega_{i}=1$, i.e. $\hat{M}_{i}=0$, but can be described through an expansion in $\omega_{i}$ around $\omega_{i}=0$. To this end we introduce the operators

$$
\mathbf{M}_{J}^{(i)}=\left.\frac{1}{J !}\left(\frac{\mathrm{d}}{\mathrm{d} \omega_{i}}\right)^{J}\right|_{\omega_{i}=0} \quad \text { and } \quad \mathbf{M}_{\left[0, X_{i}\right]}^{(i)}=\sum_{J=0}^{X_{i}} \mathbf{M}_{J}^{(i)}
$$

Note that loop amplitudes with $\omega_{i} \neq 1$ depend only on the squared mass $\hat{M}_{i}^{2}$. Thus the operator $\mathbf{M}_{J}^{(i)}$ yields zero for odd values of $J$, while for even values of $J$ it generates terms of relative order $\left(M / \bar{q}_{i}\right)^{J}$. In practice, renormalisable theories require expansions only up to order $X_{i} \leq 2$, and, once all $\left\{p_{i a}, m_{i a}\right\}$ have been set equal to zero in the denominators as a result of (A.22), only the two following trivial $\hat{M}_{i}$ expansions are required,

$$
\begin{array}{cl}
\mathbf{M}_{\left[0, X_{i}\right]}^{(i)} \frac{1}{\left(\bar{q}_{i}^{2}-\hat{M}_{i}^{2}\right)^{P_{i}}}=\frac{1}{\left(\bar{q}_{i}^{2}-M^{2}\right)^{P_{i}}} & \text { for } \quad X_{i} \leq 1, \\
\mathbf{M}_{[0,2]}^{(i)} \frac{1}{\left(\bar{q}_{i}^{2}-\hat{M}_{i}^{2}\right)^{P_{i}}}=\frac{1}{\left(\bar{q}_{i}^{2}-M^{2}\right)^{P_{i}}}\left(1-P_{i} \frac{M^{2}}{\bar{q}_{i}^{2}-M^{2}}\right) & \text { for } \quad X_{i}=2 .
\end{array}
$$

Here $1 /\left(\bar{q}_{i}^{2}-\hat{M}_{i}^{2}\right)^{P_{i}}$ is a tadpole denominator that results from the $\left\{p_{i a}, m_{i a}\right\}$ expansion of a certain chain $\mathcal{C}_{i}$ with auxiliary mass (A.23).

In order to express the expansion of a generic loop chain in terms of the operators (A.20) and (A.24), let us define the modified loop chain

$$
\overline{\mathcal{F}}^{(i)}\left(\left\{\hat{\ell}_{i a}, \hat{m}_{i a}\right\}, \hat{M}_{i}\right)=\frac{\overline{\mathcal{N}}^{(i)}\left(\left\{\hat{\ell}_{i a}, \hat{m}_{i a}\right\}\right)}{\mathcal{D}^{(i)}\left(\left\{\hat{\ell}_{i a}, \hat{m}_{i a}\right\}, \hat{M}_{i}\right)},
$$

where we explicitly indicate the dependence on $\hat{\ell}_{i a}, \hat{m}_{i a}$ and $\hat{M}_{i}$, and the modified chain denominator is defined as

$$
\mathcal{D}^{(i)}\left(\left\{\hat{\ell}_{i a}, \hat{m}_{i a}\right\}, \hat{M}_{i}\right)=\prod_{a=0}^{N_{i}-1}\left(\hat{\ell}_{i a}^{2}-\hat{m}_{i a}^{2}-\hat{M}_{i}^{2}\right),
$$

while the associated numerator in (A.26) corresponds to the usual chain numerator $\overline{\mathcal{N}}_{\bar{\alpha}_{i}}^{(i)}\left(\bar{q}_{i}\right)$ with $p_{i a} \rightarrow \hat{p}_{i a}, m_{i a} \rightarrow \hat{m}_{i a}$ and with the multi-index $\bar{\alpha}_{i}$ kept implicit. For $\lambda_{i}=\omega_{i}=1$ the modified chain (A.26) is equivalent to (A.6), while applying $\mathbf{T}_{K}^{(i)} \mathbf{M}_{J}^{(i)}$ to (A.26) generates a massive tadpole chain of order $K$ in $\left\{p_{i a} / \bar{q}_{i}, m_{i a} / \bar{q}_{i}\right\}$ and order $J$ in $M / \bar{q}_{i}$. Thus the truncated tadpole expansion (A.17) can be generated by applying (A.26) to all $\mathbf{T}_{K}^{(i)} \mathbf{M}_{J}^{(i)}$ combinations with $K+J \leq X_{i}$, i.e.

$$
\mathbf{S}_{X_{i}}^{(i)} \overline{\mathcal{F}}^{(i)}\left(\bar{q}_{i}\right)=\sum_{J=0}^{X_{i}} \sum_{K=0}^{X_{i}-J} \mathbf{T}_{K}^{(i)} \mathbf{M}_{J}^{(i)} \overline{\mathcal{F}}^{(i)}\left(\left\{\hat{\ell}_{i a}, \hat{m}_{i a}\right\}, \hat{M}_{i}^{2}\right) .
$$

In order to arrive at a more efficient expansion, we first observe that the expansions in $\lambda_{i}$ and $\omega_{i}$ can be decoupled from each other by replacing

$$
\sum_{J=0}^{X_{i}} \sum_{K=0}^{X_{i}-J} \mathbf{T}_{K}^{(i)} \mathbf{M}_{J}^{(i)} \rightarrow \mathbf{T}_{\left[0, X_{i}\right]}^{(i)} \mathbf{M}_{\left[0, X_{i}\right]}^{(i)}
$$


Such a modified expansion generates unnecessary extra terms up to order $\left(1 / \bar{q}_{i}\right)^{2 X_{i}}$ for each chain. However it makes it possible to combine the $\mathbf{T}_{\left[0, X_{i}\right]}^{(i)}$ expansions of all chains into a global expansion in $\left\{p_{i a}, m_{i a}\right\}$ that extends to the full integral, including also the vertices that connect the various chains. In this way, exploiting the fact that $\delta \mathcal{R}_{2}$ terms are homogeneous polynomials of order $X$ in $\left\{p_{i a}, m_{i a}\right\}$, all contributions of lower and higher order can be discarded. Thus one can replace

$$
\prod_{i} \mathbf{T}_{\left[0, X_{i}\right]}^{(i)} \mathbf{M}_{\left[0, X_{i}\right]}^{(i)} \rightarrow \mathbf{T}_{X} \prod_{i} \mathbf{M}_{\left[0, X_{i}\right]}^{(i)}
$$

where $\mathbf{T}_{X}$ extracts terms of fixed order $X$ in all $\left\{p_{i a}, m_{i a}\right\}$. More precisely, one can apply a global rescaling parameter $\lambda$ to all momenta and masses inside loop chains, connecting vertices and counterterms,

$$
\hat{p}_{i a}=\lambda p_{i a}, \quad \hat{m}_{i a}=\lambda m_{i a} \quad \forall i, a,
$$

and define the expansion operator

$$
\mathbf{T}_{X}=\left.\frac{1}{K !}\left(\frac{\mathrm{d}}{\mathrm{d} \lambda}\right)^{K}\right|_{\lambda=0}
$$

With these conventions one can define the optimised tadpole expansion ${ }^{10}$

$$
\overline{\mathcal{A}}_{k, \Gamma_{\mathrm{tad}}}=\mathbf{T}_{X}\left(\prod_{i} \mathbf{M}_{\left[0, X_{i}\right]}^{(i)}\right) \overline{\mathcal{A}}_{k, \Gamma}
$$

where $\overline{\mathcal{A}}_{k, \Gamma}$ is the amplitude of a generic $k$-loop diagram, and the product includes all relevant loop chains, i.e. one chain at one loop and three chains at two loops. For oneloop diagrams with a counterterm insertion the expansion should be carried out as for bare one-loop diagrams, applying $\mathbf{T}_{X}$ also to the momentum and mass dependence of the counterterm.

When using the optimised tadpole expansion (A.33) for the calculation of $\delta \mathcal{R}_{2}$ terms, on the r.h.s. of (A.13) one should apply a global Taylor expansion $\mathbf{T}_{X}$, where $X=X(\Gamma)$ is the global degree of divergence of the two-loop diagram at hand, and replace $\mathbf{S}_{X_{i}}^{(i)}$ by $\mathbf{M}_{X_{i}}^{(i)}$, where $X_{i}=X_{i}(\Gamma)$ is defined in (A.11). More explicitly, for an individual two-loop diagram the above expansion amounts to the following operations.

1. Rescale all external masses and internal momenta according to (A.31) and insert the auxiliary mass term $\hat{M}_{i}^{2}$ in every propagator denominator that depends on the loop momentum $\bar{q}_{i}$.

2. Apply the operator $\mathbf{T}_{X}$, which selects terms of fixed total order $X=X(\Gamma)$ in $\left\{p_{i a}, m_{i a}\right\}$, at the level of the full two-loop diagram. This yields tadpole integrals with denominators of the form $\prod_{i}\left(\bar{q}_{i}^{2}-\hat{M}_{i}^{2}\right)^{P_{i}}$, where $P_{i} \in\left[N_{i}, N_{i}+X\right]$.

\footnotetext{
${ }^{10}$ Here and in the following is implicitly understood that the parameters of the amplitude $\overline{\mathcal{A}}_{k, \Gamma}$ on the r.h.s. should be rescaled according to (A.23) and (A.32) before applying the $\mathbf{T}_{X}$ and $\mathbf{M}_{\left[0, X_{i}\right]}^{(i)}$ operators.
} 
3. Apply the auxiliary-mass expansions $\prod_{i} \mathbf{M}_{\left[0, X_{i}\right]}$ using (A.25).

The same procedure should be used for one-loop diagrams with counterterm insertions. In that case, according to (A.16), the order of the $\mathbf{M}_{\left[0, X_{i}\right]}^{(i)}$ expansions is simply $X_{i}=X(\Gamma)$. Note that steps 2 and 3 of the above algorithm may be inverted. Alternatively, they may be implemented by means of the recursive tadpole decomposition (A.1) with subsequent selection of terms of total order $X(\Gamma)$ in $\left\{p_{i a}, m_{i a}\right\}$ and from order zero to $X_{i}$ in $\hat{M}_{i}$.

Contrary to the naive tadpole expansion discussed in sections A.1-A.2, the optimised expansion (A.33) and its further simplification described in section A.4 are invariant w.r.t. shifts of the loop momenta. This is demonstrated in section A.5.

\section{A.4 Taylor expansion with auxiliary one-loop counterterms}

In this section we outline an alternative tadpole expansion method that is widely used in multi-loop calculations of beta functions [49-51]. This method makes it possible to isolate local divergences without applying any auxiliary-mass expansion. It can be understood by starting from the expansion (A.33) and disentangling the effects of the $\mathbf{T}_{X}$ and $\mathbf{M}_{X_{i}}^{(i)}$ operators. Applying only the $\mathbf{T}_{X}$ expansion to a generic $k$-loop integral results into combinations of tadpole integrals of type

$$
\mathbf{T}_{X}\left(\prod_{i} \mathbf{M}_{\left[0, X_{i}\right]}^{(i)}\right) \overline{\mathcal{A}}_{k, \Gamma}=\left(\prod_{i} \mathbf{M}_{\left[0, X_{i}\right]}^{(i)}\right) \sum_{\vec{P}} \int \prod_{i} \mathrm{~d} \bar{q}_{i} \frac{\mathcal{T}_{\vec{P}}\left(\left\{\bar{q}_{k}, p_{k a}, m_{k a}\right\}\right)}{\prod_{j}\left(\bar{q}_{j}^{2}-\hat{M}_{j}\right)^{P_{i}}}
$$

where $\vec{P}=\left(P_{1}, \ldots\right)$ describes the denominator powers of the various loop chains. The numerators $\mathcal{T}_{\vec{P}}\left(\left\{\bar{q}_{k}, p_{k a}, m_{k a}\right\}\right)$, which result from the $\mathbf{T}_{X}$ expansion of the original integral, is a polynomials of homogeneous degree $X$ in $\left\{p_{i a}, m_{i a}\right\}$. Moreover, in the case of amputated 1PI diagrams, the superficial degree of divergence $X$ corresponds to the mass dimension of the diagram. Therefore the massive tadpole integrals on the r.h.s. of (A.34) must have vanishing global degree of divergence. Let us now consider the effect of the auxiliary-mass expansions. The leading contribution

$$
\mathbf{M}_{0}=\prod_{i} \mathbf{M}_{0}^{(i)}
$$

which amounts to inserting by hand a mass term $\hat{M}_{i}=M$ in all denominators, does not modify the superficial degree of divergence. Instead, the remnant part

$$
\Delta \mathbf{M}=\prod_{i} \mathbf{M}_{\left[0, X_{i}\right]}^{(i)}-\mathbf{M}_{0},
$$

is either vanishing (when $X_{i}<2$ for all $i$ ) or contains at least one auxiliary-mass derivative, which results in a $M^{2} / \bar{q}_{i}^{2}$ suppression. This implies that such terms have a negative degree of superficial divergence. Therefore they are only relevant for a correct description of all subdivergences, i.e. in order to guarantee (3.21)-(3.22), but they do not contribute to $\delta \mathcal{R}_{2, \Gamma}$.

This implies that two-loop rational terms can be computed using the minimal expansion

$$
\overline{\mathcal{A}}_{k, \Gamma_{\text {tad }}}=\mathbf{T}_{X} \mathbf{M}_{0} \overline{\mathcal{A}}_{k, \Gamma} .
$$


With this approach the formula for the calculation of $\delta \mathcal{R}_{2}$ terms becomes

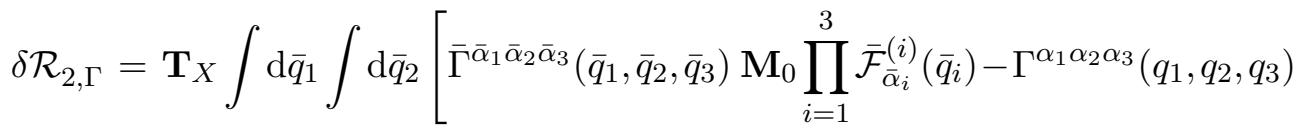

$$
\begin{aligned}
& \left.\times \mathbf{M}_{0} \prod_{i=1}^{3} \mathcal{F}_{\alpha_{i}}^{(i)}\left(q_{i}\right)\right]_{q_{3}=-q_{1}-q_{2}}+\sum_{i=1}^{3} \mathbf{T}_{X} \int \mathrm{d} \bar{q}_{i}\left[\delta Z_{1, \gamma_{\mathrm{tad}, i}}^{\bar{\alpha}_{i}}\left(\bar{q}_{i}, M\right) \mathbf{M}_{0} \overline{\mathcal{F}}_{\bar{\alpha}_{i}}^{(i)}\left(\bar{q}_{i}\right)\right. \\
& \left.-\left(\delta Z_{1, \gamma_{\mathrm{tad}, i}}^{\alpha_{i}}\left(q_{i}, M\right)+\delta \tilde{Z}_{1, \gamma_{\mathrm{tad}, i}}^{\alpha_{i}}\left(\tilde{q}_{i}\right)+\delta \mathcal{R}_{1, \gamma_{\mathrm{tad}, i}}^{\alpha_{i}}\left(q_{i}, M\right)\right) \mathbf{M}_{0} \mathcal{F}_{\alpha_{i}}^{(i)}\left(q_{i}\right)\right],
\end{aligned}
$$

where it is understood that, before applying $\mathbf{T}_{X}$, all physical masses and momenta should be rescaled according to (A.31). Since the omission of the higher-order terms (A.36) modifies all quadratic subdivergences, the various UV and rational one-loop counterterms in (A.38) should be adapted to the modified one-loop subdiagrams $\mathbf{M}_{0} \overline{\mathcal{A}}_{1, \gamma_{i}}$. For instance, the required UV counterterm in $D_{\mathrm{n}}=D$ is

$$
\delta Z_{1, \gamma_{\mathrm{tad}, i}}^{\bar{\alpha}_{i}}\left(\bar{q}_{i}, M\right)=-\mathbf{K} \mathbf{M}_{0} \overline{\mathcal{A}}_{1 \gamma_{i}}^{\bar{\alpha}_{i}}\left(\bar{q}_{i}\right) .
$$

Assuming that subdivergences are at most quadratic, (A.39) is related to the standard UV counterterm through

$$
\delta Z_{1, \gamma_{\mathrm{tad}, i}}^{\bar{\alpha}_{i}}\left(\bar{q}_{i}, M\right)=\delta Z_{1, \gamma_{i}}^{\bar{\alpha}_{i}}\left(\bar{q}_{i}\right)+\mathbf{K}\left[M^{2} \frac{\mathrm{d}}{\mathrm{d} M^{2}}\left(\mathbf{M}_{0} \overline{\mathcal{A}}_{1 \gamma_{i}}^{\bar{\alpha}_{i}}\left(\bar{q}_{i}\right)\right)\right] .
$$

The $M$-dependent term on the r.h.s. contributes only in the presence of quadratic subdivergences and can be treated as an extra auxiliary counterterm. Similar $M$-dependent terms need to be included also in the rational terms $\delta \mathcal{R}_{1, \gamma_{\text {tad }, i}}^{\alpha_{i}}\left(q_{i}, M\right)$, while in renormalisable theories $\delta \tilde{Z}_{1, \gamma_{\text {tad }, i}}^{\alpha_{i}}\left(\tilde{q}_{i}\right)$ is independent of $M$, since the full $M$-dependence of the UV counterterm in $D_{\mathrm{n}}=4$ can be absorbed into $\delta Z_{1, \gamma_{\mathrm{tad}, i}}^{\alpha_{i}}\left(\tilde{q}_{i}, M\right)$.

\section{A.5 Invariance with respect to shifts of the loop momenta}

As discussed in section A.2, tadpole expansions can depend on the parametrisation of loop integrals, i.e. shifts of the loop momentum can lead to a different result. For this reason, when computing $\delta \mathcal{R}_{2}$ terms care must be taken that parametrisation-dependent terms do cancel out as they should. To this end, when using the naive expansions of sections A.1-A.2, two-loop diagrams and related one-loop counterterm insertions should be parametrised in the same way. On the contrary, as demonstrated in the following, when using the optimised tadpole expansions (A.33) and (A.37) the parametrisations of all loop integrals can be chosen independently from one another.

As a starting point, let us consider the interplay of a loop-momentum shift $\bar{q}_{i} \rightarrow \bar{q}_{i}+\Delta p_{i}$ with the expansion (A.33) of a generic one-loop integral,

$$
\mathbf{T}_{X} \mathbf{M}_{\left[0, X_{i}\right]}^{(i)} \overline{\mathcal{A}}_{1}\left(\left\{p_{i a}+\Delta p_{i}, m_{i a}\right\}\right)=\left.\int \mathrm{d} \bar{q}_{i} \tilde{\mathbf{T}}_{X} \mathbf{M}_{\left[0, X_{i}\right]}^{(i)} \overline{\mathcal{F}}\left(\left\{\hat{\ell}_{i a}, \hat{m}_{i a}\right\}, \hat{M}_{i}^{2}\right)\right|_{\hat{\ell}_{i a}=\bar{q}_{i}+\Delta \hat{p}_{i}+\hat{p}_{i a}},
$$


where $X$ is the superficial degree of divergence of the diagram at hand, while $X_{i}$ may have arbitrary values, and the case $X_{i}=0$ corresponds to the expansion (A.37). The momentum shift $\Delta p_{i}$ is a certain combination of the external momenta $p_{i a}$. Thus $\Delta p_{i}$ should undergo the same $\lambda$-rescaling (A.31) and expansion (A.32) as the original external momenta. However, for a transparent bookkeeping of the dependence on $\Delta p_{i}$ we introduce an independent rescaling

$$
\Delta \hat{p}_{i}=\tilde{\lambda} \Delta p_{i}
$$

and on the r.h.s. of (A.41) we carry out a simultaneous expansion in $\lambda$ and $\tilde{\lambda}$, which is embodied in the operator

$$
\tilde{\mathbf{T}}_{X}=\left.\frac{1}{X !}\left(\frac{\mathrm{d}}{\mathrm{d} \tilde{\lambda}}+\frac{\mathrm{d}}{\mathrm{d} \lambda}\right)^{X}\right|_{\lambda=\tilde{\lambda}=0}=\left.\left.\sum_{K=0}^{X} \frac{1}{K !(X-K) !}\left(\frac{\mathrm{d}}{\mathrm{d} \tilde{\lambda}}\right)^{K}\right|_{\tilde{\lambda}=0}\left(\frac{\mathrm{d}}{\mathrm{d} \lambda}\right)^{X-K}\right|_{\lambda=0},
$$

where

$$
\left.\left(\frac{\mathrm{d}}{\mathrm{d} \tilde{\lambda}}\right)^{K}\right|_{\tilde{\lambda}=0}=\left.\left(\Delta p_{i}^{\mu} \sum_{a} \frac{\partial}{\partial \hat{\ell}_{i a}^{\mu}}\right)^{K}\right|_{\tilde{\lambda}=0}=\left.\left(\Delta p_{i}^{\mu} \frac{\partial}{\partial \bar{q}_{i}^{\mu}}\right)^{K}\right|_{\tilde{\lambda}=0} .
$$

Since $\tilde{\lambda}, \lambda$ and $\omega_{i}$ are independent expansion parameters, the corresponding derivatives commute. Thus combining (A.32), (A.41) and (A.43)-(A.44) we can write

$$
\begin{aligned}
& \mathbf{T}_{X} \mathbf{M}_{\left[0, X_{i}\right]}^{(i)} \overline{\mathcal{A}}_{1}\left(\left\{p_{i a}+\Delta p_{i}, m_{i a}\right\}\right)= \\
& \quad=\sum_{K=0}^{X} \int \mathrm{d} \bar{q}_{i} \frac{1}{K !}\left(\Delta p_{i}^{\mu} \frac{\partial}{\partial \bar{q}_{i}^{\mu}}\right)^{K}\left\{\left.\mathbf{T}_{X-K} \mathbf{M}_{\left[0, X_{i}\right]}^{(i)} \overline{\mathcal{F}}\left(\left\{\hat{\ell}_{k}, \hat{m}_{k}\right\}, \hat{M}^{2}\right)\right|_{\hat{\ell}_{k}=\bar{q}_{i}+\hat{p}_{k}}\right\} .
\end{aligned}
$$

Here all terms with $K>0$ on the r.h.s. integrate to zero since the corresponding integrands have the form of a total $\partial / \partial \bar{q}_{i}^{\mu}$ derivative, while the remaining $K=0$ term corresponds to the expansion of the original integral with $\Delta p_{i}=0$, i.e.

$$
\mathbf{T}_{X} \mathbf{M}_{\left[0, X_{i}\right]}^{(i)} \overline{\mathcal{A}}_{1}\left(\left\{p_{i a}+\Delta p_{i}, m_{i a}\right\}\right)=\mathbf{T}_{X} \mathbf{M}_{\left[0, X_{i}\right]}^{(i)} \overline{\mathcal{A}}_{1}\left(\left\{p_{i a}, m_{i a}\right\}\right) .
$$

This demonstrates that, when applied to one-loop integrals, the tadpole expansions (A.33) and (A.37) are invariant w.r.t. shifts of the loop momentum. Along similar lines one can show that this holds also beyond one loop.

\section{B Renormalisation constants in the $\overline{\mathrm{MS}}$ scheme}

For convenience of the reader, in this appendix we list the explicit expressions of the renormalisation constants that enter the Yang-Mills Lagrangian (5.2) for the case of the $\overline{\mathrm{MS}}$ scheme. Similarly as in section 5 we adopt the Feynman gauge, and we use the convention (5.7) for the perturbative expansion of the various renormalisation constants. In the 
$\overline{\mathrm{MS}}$ scheme, the rescaling factor $S$ that enters $t=S \mu_{0}^{2} / \mu_{\mathrm{R}}^{2}$ in (5.7) is defined through (4.30) and, according to (4.32), the scale-independent parts of the renormalisation constants are the same as in the MS scheme,

$$
\delta \hat{\mathcal{Z}}_{k, \chi}^{(\mathrm{MS})}=\delta \hat{\mathcal{Z}}_{k, \chi}^{(\overline{\mathrm{MS}})}=\delta \hat{\mathcal{Z}}_{k, \chi}^{\left(\mathrm{MS}_{0}\right)} .
$$

The gauge-fixing term does not receive any finite renormalisation in the $\overline{\mathrm{MS}}$ scheme, i.e. $\mathcal{Z}_{\mathrm{gp}}=1$, while the scale-independent parts of the other renormalisation constants read

$$
\begin{aligned}
& \delta \hat{\mathcal{Z}}_{1, \alpha}^{(\mathrm{MS})}=\left(-\frac{11}{3} C_{\mathrm{A}}+\frac{4}{3} T_{\mathrm{F}} n_{\mathrm{f}}\right) \varepsilon^{-1} \\
& \delta \hat{\mathcal{Z}}_{2, \alpha}^{(\mathrm{MS})}=\left(\frac{121}{9} C_{\mathrm{A}}^{2}-\frac{88}{9} T_{\mathrm{F}} n_{\mathrm{f}} C_{\mathrm{A}}+\frac{16}{9} T_{\mathrm{F}}^{2} n_{\mathrm{f}}^{2}\right) \varepsilon^{-2}-\left[\frac{17}{3} C_{\mathrm{A}}^{2}-T_{\mathrm{F}} n_{\mathrm{f}}\left(\frac{10}{3} C_{\mathrm{A}}+2 C_{\mathrm{F}}\right)\right] \varepsilon^{-1} \\
& \delta \hat{\mathcal{Z}}_{1, f}^{(\mathrm{MS})}=-C_{\mathrm{F}} \varepsilon^{-1} \\
& \delta \hat{\mathcal{Z}}_{2, f}^{(\mathrm{MS})}=\left(\frac{1}{2} C_{\mathrm{F}}^{2}+C_{\mathrm{A}} C_{\mathrm{F}}\right) \varepsilon^{-2}+\left(\frac{3}{4} C_{\mathrm{F}}^{2}-\frac{17}{4} C_{\mathrm{A}} C_{\mathrm{F}}+T_{\mathrm{F}} n_{\mathrm{f}} C_{\mathrm{F}}\right) \varepsilon^{-1} \\
& \delta \hat{\mathcal{Z}}_{1, m_{f}}^{(\mathrm{MS})}=-3 C_{\mathrm{F}} \varepsilon^{-1} \\
& \delta \hat{\mathcal{Z}}_{2, m_{f}}^{(\mathrm{MS})}=C_{\mathrm{F}}\left[\left(\frac{9}{2} C_{\mathrm{F}}+\frac{11}{2} C_{\mathrm{A}}-2 T_{\mathrm{F}} n_{\mathrm{f}}\right) \varepsilon^{-2}-\left(\frac{3}{4} C_{\mathrm{F}}+\frac{97}{12} C_{\mathrm{A}}-\frac{5}{3} T_{\mathrm{F}} n_{\mathrm{f}}\right) \varepsilon^{-1}\right] \\
& \delta \hat{\mathcal{Z}}_{1, A}^{(\mathrm{MS})}=\left(\frac{5}{3} C_{\mathrm{A}}-\frac{4}{3} T_{\mathrm{F}} n_{\mathrm{f}}\right) \varepsilon^{-1} \\
& \delta \hat{\mathcal{Z}}_{2, A}^{(\mathrm{MS})}=\left(-\frac{25}{12} C_{\mathrm{A}}^{2}+\frac{5}{3} T_{\mathrm{F}} n_{\mathrm{f}} C_{\mathrm{A}}\right) \varepsilon^{-2}+\left[\frac{23}{8} C_{\mathrm{A}}^{2}-T_{\mathrm{F}} n_{\mathrm{f}}\left(\frac{5}{2} C_{\mathrm{A}}+2 C_{\mathrm{F}}\right)\right] \varepsilon^{-1} \\
& \delta \hat{\mathcal{Z}}_{1, u}^{(\mathrm{MS})}=\frac{C_{\mathrm{A}}}{2} \varepsilon^{-1}, \\
& \delta \hat{\mathcal{Z}}_{2, u}^{(\mathrm{MS})}=\left(-C_{\mathrm{A}}^{2}+\frac{1}{2} T_{\mathrm{F}} n_{\mathrm{f}} C_{\mathrm{A}}\right) \varepsilon^{-2}+\left(\frac{49}{48} C_{\mathrm{A}}^{2}-\frac{5}{12} T_{\mathrm{F}} n_{\mathrm{f}} C_{\mathrm{A}}\right) \varepsilon^{-1} .
\end{aligned}
$$

These renormalisation constants have been computed in the same framework as the rational terms and agree with those in the literature, which have been available for a long time [52-54]. Specific results for $\mathrm{SU}(\mathrm{N})$ and U(1) gauge theories can be obtained by applying the substitutions in table 5.2.

Open Access. This article is distributed under the terms of the Creative Commons Attribution License (CC-BY 4.0), which permits any use, distribution and reproduction in any medium, provided the original author(s) and source are credited.

\section{References}

[1] S. Pozzorini, H. Zhang and M.F. Zoller, Rational Terms of UV Origin at Two Loops, JHEP 05 (2020) 077 [arXiv:2001.11388] [INSPIRE].

[2] G. 't Hooft and M.J.G. Veltman, Regularization and Renormalization of Gauge Fields, Nucl. Phys. B 44 (1972) 189 [INSPIRE]. 
[3] Z. Bern and D.A. Kosower, The Computation of loop amplitudes in gauge theories, Nucl. Phys. B 379 (1992) 451 [INSPIRE].

[4] W.B. Kilgore, The Four Dimensional Helicity Scheme Beyond One Loop, Phys. Rev. D 86 (2012) 014019 [arXiv:1205.4015] [INSPIRE].

[5] W. Siegel, Supersymmetric Dimensional Regularization via Dimensional Reduction, Phys. Lett. B 84 (1979) 193 [INSPIRE].

[6] A. Signer and D. Stöckinger, Factorization and regularization by dimensional reduction, Phys. Lett. B 626 (2005) 127 [hep-ph/0508203] [INSPIRE].

[7] A.L. Cherchiglia, M. Sampaio and M.C. Nemes, Systematic Implementation of Implicit Regularization for Multi-Loop Feynman Diagrams, Int. J. Mod. Phys. A 26 (2011) 2591 [arXiv: 1008.1377] [INSPIRE].

[8] R. Pittau, A four-dimensional approach to quantum field theories, JHEP 11 (2012) 151 [arXiv: 1208.5457] [INSPIRE].

[9] B. Page and R. Pittau, Two-loop off-shell QCD amplitudes in FDR, JHEP 11 (2015) 183 [arXiv: 1506. 09093] [INSPIRE].

[10] R.A. Fazio, P. Mastrolia, E. Mirabella and W.J. Torres Bobadilla, On the Four-Dimensional Formulation of Dimensionally Regulated Amplitudes, Eur. Phys. J. C 74 (2014) 3197 [arXiv: 1404.4783] [INSPIRE].

[11] G. Ossola, C.G. Papadopoulos and R. Pittau, On the Rational Terms of the one-loop amplitudes, JHEP 05 (2008) 004 [arXiv: 0802.1876] [INSPIRE].

[12] W.T. Giele, Z. Kunszt and K. Melnikov, Full one-loop amplitudes from tree amplitudes, JHEP 04 (2008) 049 [arXiv: 0801.2237] [InSPIRE].

[13] S. Abreu, F. Febres Cordero, H. Ita, B. Page and M. Zeng, Planar Two-Loop Five-Gluon Amplitudes from Numerical Unitarity, Phys. Rev. D 97 (2018) 116014 [arXiv:1712.03946] [INSPIRE].

[14] D.E. Soper, Techniques for QCD calculations by numerical integration, Phys. Rev. D 62 (2000) 014009 [hep-ph/9910292] [INSPIRE].

[15] Z. Nagy and D.E. Soper, General subtraction method for numerical calculation of one loop QCD matrix elements, JHEP 09 (2003) 055 [hep-ph/0308127] [INSPIRE].

[16] S. Catani, T. Gleisberg, F. Krauss, G. Rodrigo and J.-C. Winter, From loops to trees by-passing Feynman's theorem, JHEP 09 (2008) 065 [arXiv:0804.3170] [INSPIRE].

[17] G.F.R. Sborlini, F. Driencourt-Mangin, R. Hernandez-Pinto and G. Rodrigo, Four-dimensional unsubtraction from the loop-tree duality, JHEP 08 (2016) 160 [arXiv: 1604.06699] [INSPIRE].

[18] J.J. Aguilera-Verdugo et al., Open Loop Amplitudes and Causality to All Orders and Powers from the Loop-Tree Duality, Phys. Rev. Lett. 124 (2020) 211602 [arXiv:2001.03564] [INSPIRE].

[19] S. Becker, C. Reuschle and S. Weinzierl, Numerical NLO QCD calculations, JHEP 12 (2010) 013 [arXiv: 1010.4187] [INSPIRE].

[20] S. Becker and S. Weinzierl, Direct numerical integration for multi-loop integrals, Eur. Phys. J. C 73 (2013) 2321 [arXiv:1211.0509] [INSPIRE]. 
[21] C. Anastasiou and G. Sterman, Removing infrared divergences from two-loop integrals, JHEP 07 (2019) 056 [arXiv: 1812.03753] [INSPIRE].

[22] Z. Capatti, V. Hirschi, D. Kermanschah, A. Pelloni and B. Ruijl, Numerical Loop-Tree Duality: contour deformation and subtraction, JHEP 04 (2020) 096 [arXiv: 1912.09291] [INSPIRE].

[23] P. Draggiotis, M.V. Garzelli, C.G. Papadopoulos and R. Pittau, Feynman Rules for the Rational Part of the QCD 1-loop amplitudes, JHEP 04 (2009) 072 [arXiv:0903.0356] [INSPIRE].

[24] M.V. Garzelli, I. Malamos and R. Pittau, Feynman rules for the rational part of the Electroweak 1-loop amplitudes, JHEP 01 (2010) 040 [Erratum ibid. 10 (2010) 097] [arXiv:0910.3130] [INSPIRE].

[25] R. Pittau, Primary Feynman rules to calculate the epsilon-dimensional integrand of any 1-loop amplitude, JHEP 02 (2012) 029 [arXiv:1111.4965] [INSPIRE].

[26] F. Buccioni et al., OpenLoops 2, Eur. Phys. J. C 79 (2019) 866 [arXiv:1907.13071] [INSPIRE].

[27] A. Denner, J.-N. Lang and S. Uccirati, Recola2: REcursive Computation of One-Loop Amplitudes 2, Comput. Phys. Commun. 224 (2018) 346 [arXiv:1711.07388] [INSPIRE].

[28] A. van Hameren, C.G. Papadopoulos and R. Pittau, Automated one-loop calculations: A Proof of concept, JHEP 09 (2009) 106 [arXiv:0903.4665] [INSPIRE].

[29] V. Hirschi, R. Frederix, S. Frixione, M.V. Garzelli, F. Maltoni and R. Pittau, Automation of one-loop QCD corrections, JHEP 05 (2011) 044 [arXiv: 1103.0621] [INSPIRE].

[30] N.N. Bogoliubov and O.S. Parasiuk, On the Multiplication of the causal function in the quantum theory of fields, Acta Math. 97 (1957) 227 [INSPIRE].

[31] K. Hepp, Proof of the Bogolyubov-Parasiuk theorem on renormalization, Commun. Math. Phys. 2 (1966) 301 [INSPIRE].

[32] W. Zimmermann, Convergence of Bogolyubov's method of renormalization in momentum space, Commun. Math. Phys. 15 (1969) 208 [INSPIRE].

[33] W.E. Caswell and A.D. Kennedy, A simple approach to renormalization theory, Phys. Rev. D 25 (1982) 392 [INSPIRE].

[34] A. Bredenstein, A. Denner, S. Dittmaier and S. Pozzorini, $N L O$ QCD corrections to $t \bar{t} b \bar{b}$ production at the LHC: 1. Quark-antiquark annihilation, JHEP 08 (2008) 108 [arXiv: 0807.1248] [INSPIRE].

[35] F. del Aguila and R. Pittau, Recursive numerical calculus of one-loop tensor integrals, JHEP 07 (2004) 017 [hep-ph/0404120] [INSPIRE].

[36] G. Ossola, C.G. Papadopoulos and R. Pittau, Reducing full one-loop amplitudes to scalar integrals at the integrand level, Nucl. Phys. B 763 (2007) 147 [hep-ph/0609007] [InSPIRE].

[37] A.L. Kataev and M.D. Vardiashvili, Scheme Dependence of the Perturbative Series for a Physical Quantity in the $g \phi^{4}$ Theory, Phys. Lett. B 221 (1989) 377 [Erratum ibid. 241 (1990) 644] [INSPIRE].

[38] K.G. Chetyrkin and M. F. Zoller, GEFICOM, private code.

[39] P. Nogueira, Automatic Feynman graph generation, J. Comput. Phys. 105 (1993) 279. 
[40] T. Seidensticker, Automatic application of successive asymptotic expansions of Feynman diagrams, in 6th International Workshop on New Computing Techniques in Physics Research 5 (1999), hep-ph/9905298 [INSPIRE].

[41] R. Harlander, T. Seidensticker and M. Steinhauser, Complete corrections of Order alpha $\alpha_{s}$ to the decay of the $Z$ boson into bottom quarks, Phys. Lett. B 426 (1998) 125 [hep-ph/9712228] [INSPIRE].

[42] J.A.M. Vermaseren, New features of FORM, math-ph/0010025 [INSPIRE].

[43] M. Tentyukov and J.A.M. Vermaseren, The Multithreaded version of FORM, Comput. Phys. Commun. 181 (2010) 1419 [hep-ph/0702279] [INSPIRE].

[44] M. Steinhauser, MATAD: A Program package for the computation of MAssive TADpoles, Comput. Phys. Commun. 134 (2001) 335 [hep-ph/0009029] [INSPIRE].

[45] T. van Ritbergen, A.N. Schellekens and J.A.M. Vermaseren, Group theory factors for Feynman diagrams, Int. J. Mod. Phys. A 14 (1999) 41 [hep-ph/9802376] [inSPIRE].

[46] G. 't Hooft, A Planar Diagram Theory for Strong Interactions, Nucl. Phys. B 72 (1974) 461 [INSPIRE].

[47] F.V. Tkachov, A Theorem on Analytical Calculability of Four Loop Renormalization Group Functions, Phys. Lett. B 100 (1981) 65 [INSPIRE].

[48] K.G. Chetyrkin and F.V. Tkachov, Integration by Parts: The Algorithm to Calculate $\beta$-functions in 4 Loops, Nucl. Phys. B 192 (1981) 159 [InSPIRE].

[49] M. Misiak and M. Münz, Two loop mixing of dimension five flavor changing operators, Phys. Lett. B 344 (1995) 308 [hep-ph/9409454] [INSPIRE].

[50] K.G. Chetyrkin, M. Misiak and M. Münz, $\beta$-functions and anomalous dimensions up to three loops, Nucl. Phys. B 518 (1998) 473 [hep-ph/9711266] [INSPIRE].

[51] M.F. Zoller, Three-loop $\beta$-function for the Higgs self-coupling, PoS LL2014 (2014) 014 [arXiv: 1407.6608] [INSPIRE].

[52] D.R.T. Jones, Two Loop Diagrams in Yang-Mills Theory, Nucl. Phys. B 75 (1974) 531 [INSPIRE].

[53] W.E. Caswell, Asymptotic Behavior of Nonabelian Gauge Theories to Two Loop Order, Phys. Rev. Lett. 33 (1974) 244 [InSPIRE].

[54] O.V. Tarasov and A.A. Vladimirov, Two Loop Renormalization of the Yang-Mills Theory in an Arbitrary Gauge, Sov. J. Nucl. Phys. 25 (1977) 585 [inSPIRE]. 